\title{
Laboratory Directed Research and Development Program FY 2006
}

March 2007 


\section{DISCLAIMER}

This document was prepared as an account of work sponsored by the United States Government. While this document is believed to contain correct information, neither the United States Government nor any agency thereof, nor The Regents of the University of California, nor any of their employees, makes any warranty, express or implied, or assumes any legal responsibility for the accuracy, completeness, or usefulness of any information, apparatus, product, or process disclosed, or represents that its use would not infringe privately owned rights. Reference herein to any specific commercial product, process, or service by its trade name, trademark, manufacturer, or otherwise, does not necessarily constitute or imply its endorsement, recommendation, or favoring by the United States Government or any agency thereof, or The Regents of the University of California. The views and opinions of authors expressed herein do not necessarily state or reflect those of the United States Government or any agency thereof or The Regents of the University of California.

Lawrence Berkeley Laboratory is an equal opportunity employer. 


\section{Report on \\ Ernest Orlando Lawrence Berkeley National Laboratory}

\section{Laboratory Directed Research and Development Program}

FY 2006

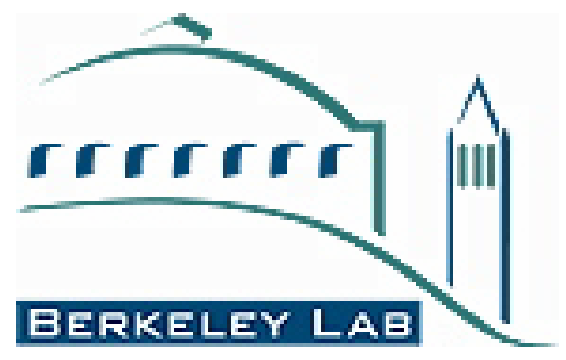

Ernest Orlando Lawrence Berkeley National Laboratory Berkeley, CA 94720
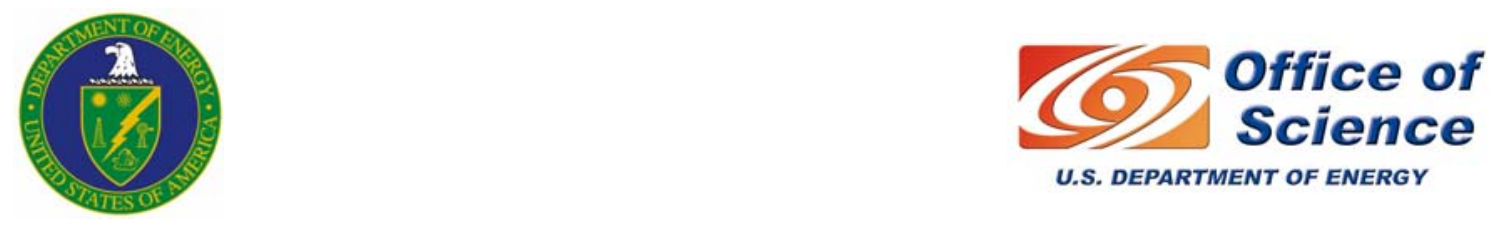

Prepared for the U.S. Department of Energy under Contract No. DE-AC03-76SF00098 



\section{Table of Contents}

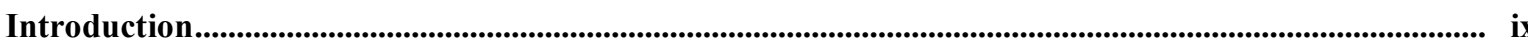

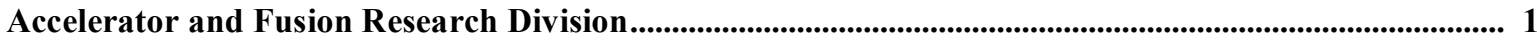

John Byrd High-Stability Beam Handling in Electron Storage Rings with

William Waldron

Applications to High Energy Colliders and Light Sources ........

Andrzej Wolski

Steven Lidia

Critical Accelerator Technologies for Advanced Light Sources ....

2

et al

Grant Logan

Enabling High Energy Density Physics at LBNL.

Jonathan Wurtele

Wim Leemans

Max Zolotorev

Low Energy Spread Electron Source.

4

Eugene Commins

Jim Oneill

Fernando Sannibale

Weishi Wan

Advanced Light Source Division

Malcolm Howells

Coherent X-Ray Diffraction Imaging (CXDI).

Anton Barty

Henry Chapman

Chris Jacobsen

Janos Kirz

Stefano Marchesini

John Spence

Uwe Weierstall

Janos Kirz

Lenless Imaging of Yeast Cells

Matthew Marcus

Measurement of Molecular Shape and Assembly Using X-ray

et al

Scattering.

Michael Martin

Zhao Hao

Left-Handed Nanoscale Meta-Materials: Towards the Optical

Eric Anderson

Domain

J. Alexander Liddle

Eli Rotenberg

Aaron Bostwick

NanoARPES: A New Detector for Nanometer-scale Electronic

Andreas Scholl

Structure Measurements

Ultrafast Magnetization Dynamics

10

Anthony Young

Jun Feng

Joachim Stöhr

Howard Padmore

Chemical Sciences Division

Musahid Ahmed

Gas Phase Studies of the Building Blocks of Life.

Christopher Chang

Oxygen Catalysis for Biomimetic Energy Conversion

Chemistry 


\section{Harvey Gould}

Stephen Leone

Daniel Neumark

John Prausnitz

Thomas Rescigno

C. William McCurdy

T. Don Tilley

Tolek Tyliszczak

David Shuh

Computing Sciences

(National Energy Research Scientific Computing Center, and Computational Research Divisions) .. 20

Electron Electric Dipole Moment Experiment in a Cold Atom

Fountain......

Time-resolved X-ray Absorption Spectroscopy of

Metalloporphyrins.

Spectroscopy and Dynamics of Pure and Doped Helium

Nanodroplets

Properties of New Ionic Liquids for Electrochemical

Applications and for Extraction of Heavy-Metal Cations from

Wastewaters

Advanced Computational Methods for Photon-Molecule

Collision Processes

New Approach for the Catalytic Conversion of Methane and

Other Inert Hydrocarbons.

Versatile Mini-Scanning Transmission X-ray Microscope (mSTXM)

Olivier Chevassut

Cryptographic Foundations for New Generation Distributed

Abdelilah Essiari

Systems

Phillip Colella

Analysis for Simulation of Nuclear Energy Systems

Software Application Infrastructure for Efficiently Managing

Large-Scale Computational Biology Experiments

Victor Markowitz

Natalia Ivanova

Integrated Microbial Community Genomes Data Management

System

Nikos Kyrpides

Phil Hugenholtz

Leonid Oliker

John Shalf

KatherineYelick

William Kramer

Enhancing Commodity Scalar Processors with Vector

Components for Increased Scientific Productivity

Advanced Computational Tools for Electric Power Systems

Bernard Lesieutre
Chao Yang
Juan Meza

Raquel Romano

Statistical Feature Modeling for Scientific Data Via Basis

Decomposition 26

Jon Wilkening

Extended First Order System Least Squares Finite Elements 27

Earth Sciences Division

Gary Andersen

Microarray Technology for Fungal Identification: Its Utility from the Environment to Homeland Security, Biomedicine, and Beyond. 28

Sally Benson

Scientific Basis for Advance Geologic Storage Technologies 29

James Bishop

Autonomous Sensors for Ocean Dissolved Organic Matter 30 
John Coates

Donald DePaolo

John Christensen

Benjamin Gilbert

Hoi-Ying Holman

Ping $\mathrm{Hu}$

Sharon Borglin

Norman Miller

Larry Dale

George Moridis

Matthew Reagan

Seiji Nakagawa

Andrea Cortis

Curtis Oldenburg

William Riley

Norman Miller

Carl Steefel

Carl Steefel
An Investigation of the Microbial Processes Involved in Electron Transfer onto the Anode of a Biological Fuel Cell

Micro-characterization and Chemical Micro-dynamics of Atmospheric Mineral Dust......................................................... 32

Behavior and Impact of Nanoparticles in the Environment.

Compositional and Functional Analysis of Cell Walls During

Metal-bacterial Interactions

The California Water and Energy System: An Approach for Addressing Future Crises

Interrelation of Global Warming and Hydrate Dissociation in

Oceanic Accumulations

New Technology for Permeability Enhancement for Natural

Gas Extraction in Tight Reservoirs

Coupled Modeling of Hydrology, Nutrient Cycling, and Vegetation: Applications to Water Quality and Water Balance.....

Biogeochemical Reaction Rates and Pathways in Porous Media... 39

Engineering Division

Peter Denes

Novel Imaging Detectors for Materials and Biology Research ......

Howard Padmore

Environmental Energy Technologies Division

Marc Fisher

Ashok Gadgil

Robert Kostecki

Venkat Srinivasan

Steve Johnson

Nitash Balsara

Jean Frechet

John Kerr

Gao Liu

Rachel Segalman

Robert Kostecki

Sanuel Mao

John Newman

Richard Russo

John Newman

Jayant Sathaye

et al.
Constraining Ammonia Emission Inventories for Control of Air

Quality

Exploring Electrochemical Arsenic Remediation of Drinking

Water for Bangladesh

Highly Efficient PLED Through Polymer Morphology Control.... 43

Surface Plasmon-Enhanced Photovoltaic Device.....

44

Analysis of High-Temperature Polymer-electrolyte Fuel-cell Phenomena.

Photons to Fuels - The Electrochemical Reduction of Carbon Dioxide to Hydrocarbons

Long-Term Global Energy Demand and Carbon Emissions

Scenarios

Genomics Division

Jonathan Eisen

Computational and Experimental Testing of Methods for Binning Sequences from Metagenomic Data 
Philip Hugenholtz Metagenomics-Enabled Analysis of Termite Hindgut

Microbiota for Biomass Conversion and Cleaner Energy............... 49

Len Pennacchio Development of Cost Effective Sequence-Based Technologies

Paul Spellman

to Identify Genomic Alterations in Cancer.

Jan-Fang Cheng

Life Sciences Division.

Manfred Auer

Susan Celniker

John Conboy

Robert Glaeser

Jian Jin

Kenneth Downing

Eva Nogales

Manfred Auer

William Jagust

David Schild

Andrew Wyrobek
Molecular Microscopy and Tomography .....

Modeling Human Disease In Drosophila melanogaster. 51

Unmasking the Human Genome Alternative Splicing Program.... 53

Improved Phase Contrast for Cryo-EM Biological Machines

and Subcellular Structure

Neuroimaging with Advanced Molecular Probes.

55

Determining if PIR51 is a Potential Tumor Suppressor Gene

Similar to $B R C A 2$...

Expression Profiling of Radiation and Cancer Susceptibility

Genes ......

Materials Sciences Division

Nitash Balsara

Electron Microscopy of Soft Matter in Two and Three

Kenneth Downing

Dimensions

Jay Groves

Christian Kisielowski

Andrew Minor

Dieter Typke

Ulrich Dahmen

Technique Development for Aberration-Corrected Microscopy

at NCEM.

Matthew Francis

Tailoring the Self Assembly of Functionalized Biomolecular

Building Blocks.

James De Yoreo

Light-activated Ion Channels and Transporters

Ehud Isacoff

Dirk Trauner

Richard Kramer

Ali Javey

Biogeochemical Reaction Rates and Pathways in Porous

Media.

Robert Kaindl

Terahertz-Frequency Conductivity and Ultrafast Optical

Yingzhong Ma

Excitations in Single-Walled Carbon Nanotubes

Carbon Based Materials for Renewable Energy

Alessandra Lanzara

Dung-Hai Lee 
Joseph Orenstein

Science and Technology of Quantum Materials

65

Ramamoorthy Ramesh

Robert Birgeneau

Alessandra Lanzara

Dung-Hai Lee

Yuri Suzuki

Ashvin Vishwanath

Alexander Pines

Robert Ritchie

Joel Ager

Antoni Tomsia

Carolyn Larabell

Gabor Somorjai

Ivo Souza

Steven Louie

Ashvin Vishwanath
Polarizer System for Microfluidics

66

Aging, Disease and the Mechanical Response of Biological

Tissues, Specifically Human Bone

Electron Glow Generated by Gas Phase Exothermic Catalytic

Reactions Using Metal-Semiconductor Nanodiodes

68

Computational and Theoretical Studies of Bulk and Nano Solid

Systems

Experimental Signatures of Fractionalized Phases and Phase

Transitions in Strongly Correlated Electronic Systems

Nuclear Science Division

Kevin Lesko

Research and Development for Double Beta Decay (DBD)

Experiments.

Margaret McMahan

New Capabilities in Nuclear Astrophysics and Radiation

Biology Using Neutrons at the 88-Inch Cyclotron 72

Larry Phair

Improved Spectroscopy of Weakly Bound States in Nuclei

Paul Vetter

Conceptual Study for a Novel Nuclear Astrophysics

Daniela Leitner

Accelerator Capability

Physical Biosciences Division

Gavin Crooks

Thomas Earnest

Daniel Fletcher

Heinz Frei

Vittal Yachandra

Jay Groves

Jan Liphardt

Eliane Trepagnier

Aleksandra Radenovic

Jan Liphardt

Michael Marletta

Jamie Cate
Statistical Dynamics of Protein Evolution 75

High-throughput Production of Proteins and Protein Complexes .. 76

Synthetic Cytoskeleton: Protrusive Structure for Reconstituted Cell Motility

Visible Light-driven Water Oxidation in Mesoporous Solids ....... 78

Magnetic Control of Spatial Organization at Bio-Membranes .......

Rapid Characterization of Microbial RNAs Using Artificial

Nanopores

Biomineral Engineering for the Production of Photonic

Structures and Light Collection Systems

Structure and Function of Intact Celluosomes ..... 


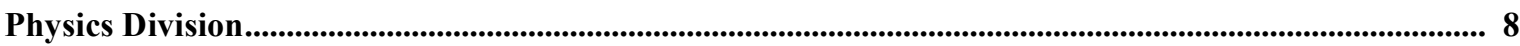

Marco Battaglia $\quad$ Advanced Monolithic Si Pixel Sensors ………………….............. 83

Maurice Garcia-Sciveres ～Nanowire Carpet Hybrid Pixel Detectors....................................... 84

Stuart Freedman Designing a Novel Reactor Neutrino Oscillation Experiment for

Karsten Heeger $\quad$ Measuring the Unknown Mixing Angle $\Theta_{13}$................................

Richard Kadel

Kam-Biu Luk

Hitoshi Murayama

New Directions for Theoretical Physics at the TeV-Scale.

86

Cross-Divisional.

Seung-Wuk Lee

Fabrication of Photovoltaic Devices Using Nanostructured

Biomaterials

87

William Lester

Development and Application of Quantum Monte Carlo (QMC)

Graham Fleming

Methods to Biological Systems.

88

Publications List 


\section{Introduction}

The Ernest Orlando Lawrence Berkeley National Laboratory (Berkeley Lab or LBNL) is a multi-program national research facility operated by the University of California for the Department of Energy (DOE). As an integral element of DOE's National Laboratory System, Berkeley Lab supports DOE's missions in fundamental science, energy resources, and environmental quality. Berkeley Lab programs advance four distinct goals for DOE and the nation:

- To perform leading multidisciplinary research in the computing sciences, physical sciences, energy sciences, biosciences, and general sciences in a manner that ensures employee and public safety and protection of the environment.

- To develop and operate unique national experimental facilities for qualified investigators.

- To educate and train future generations of scientists and engineers to promote national science and education goals.

- To transfer knowledge and technological innovations and to foster productive relationships among Berkeley Lab's research programs, universities, and industry in order to promote national economic competitiveness.

Berkeley Lab's research and the Laboratory Directed Research and Development (LDRD) program support DOE's Strategic Goals that are codified in DOE's September 2003 Strategic Plan, with a primary focus on Advancing Scientific Understanding. For that goal, the Fiscal Year (FY) 2006 LDRD projects support every one of the eight strategies described in the plan. In addition, LDRD efforts support the goals of Investing in America's Energy Future (eight of the fourteen strategies), Resolving the Environmental Legacy (three of the ten strategies), and Meeting National Security Challenges (unclassified fundamental research that supports stockpile safety and nonproliferation programs). Going forward, the program will also support the recently released 2006 DOE Strategic Plan. The LDRD supports Office of Science strategic plans, including the 20-year Scientific Facilities Plan and the Office of Science Strategic Plan. The research also supports the strategic directions periodically under review by the Office of Science Program Offices, such as strategic LDRD projects germane to new research facility concepts and new fundamental science directions.

Berkeley Lab LDRD program also play an important role in leveraging DOE capabilities for national needs. The fundamental scientific research and development conducted in the program advances the skills and technologies of importance to our Work For Others (WFO) sponsors. Among many directions, these include a broad range of health-related science and technology of interest to the National Institutes of Health, breast cancer and accelerator research supported by the Department of Defense, detector technologies that should be useful to the Department of Homeland Security, and particle detection that will be valuable to the Environmental Protection Agency.

The Berkeley Lab Laboratory Directed Research and Development Program FY2006 report is compiled from annual reports submitted by principal investigators following the close of the fiscal year. This report describes the supported projects and summarizes their accomplishments. It constitutes a part of the LDRD program planning and documentation process that includes an annual planning cycle, project selection, implementation, and review.

The Berkeley Lab LDRD program is a critical tool for directing the Laboratory's forefront scientific research capabilities toward vital, excellent, and emerging scientific challenges. The program provides the resources for Berkeley Lab scientists to make rapid and significant contributions to critical national science and technology problems. The LDRD program also advances Berkeley Lab's core competencies, foundations, and scientific capability, and permits exploration of exciting new opportunities. All projects are work in forefront areas of science and technology. Areas eligible for support include the following:

- Advanced study of hypotheses, concepts, or innovative approaches to scientific or technical problems;

- Experiments and analyses directed toward "proof of principle" or early determination of the utility of new scientific ideas, technical concepts, or devices; and

- Conception and preliminary technical analyses of experimental facilities or devices.

The LDRD program supports Berkeley Lab's mission in many ways. First, because LDRD funds can be allocated within a relatively short time frame, Berkeley Lab researchers can support the mission of the Department of Energy (DOE) and serve the needs of the nation by quickly responding to forefront scientific problems. Second, LDRD enables Berkeley Lab to attract and retain highly qualified scientists and to support their efforts to carry out worldleading research. In addition, the LDRD program also supports new projects that involve graduate students and postdoctoral fellows, thus contributing to the education mission of Berkeley Lab. 
Berkeley Lab has a formal process for allocating funds for the LDRD program. The process relies on individual scientific investigators and the scientific leadership of Berkeley Lab to identify opportunities that will contribute to scientific and institutional goals. The process is also designed to maintain compliance with DOE Orders, in particular DOE Order 413.2A, dated January 8, 2001, and its successor 413.2B, dated April 19, 2006. From year to year, the distribution of funds among the scientific program areas changes. This flexibility optimizes Berkeley Lab's ability to respond to opportunities.

Berkeley Lab LDRD policy and program decisions are the responsibility of the Laboratory Director. The Director has assigned general programmatic oversight responsibility to the Deputy Director. Administration and reporting on the LDRD program is supported by the Directorate's Office for Planning and Development. LDRD accounting procedures and financial management are consistent with the Laboratory's accounting principles and stipulations under the contract between the University of California and the Department of Energy, with accounting maintained through the Laboratory's Chief Financial Officer.

In FY2006, Berkeley Lab was authorized by DOE to establish a funding ceiling for the LDRD program of \$17.2M without General \& Administrative (G\&A) overhead or $\$ 23.9 \mathrm{M}$ with G\&A, which equates to about $3.5 \%$ and $4.9 \%$ respectively of Berkeley Lab's FY2006 projected operating and capital equipment budgets. This funding level was provided to develop new scientific ideas and opportunities and allow the Berkeley Lab Director an opportunity to initiate new directions. Budget constraints limited available resources, however, so a little more than \$18.1 M was expended for operating and \$0.5 M for capital equipment (3.84\% of actual Berkeley Lab FY2006 operating and equipment costs).

In FY2006, scientists submitted 178 proposals, requesting over $\$ 33.4 \mathrm{M}$ in total funding. Eighty-eight projects were funded, with awards ranging from $\$ 38 \mathrm{~K}$ to $\$ 1,086 \mathrm{~K}$. These projects are identified in the Table of Contents. 


\section{Accelerator and Fusion Research Division}

LB06003

High-Stability Beam Handling in Electron Storage Rings with Applications to

High Energy Colliders and Light Sources

Principal Investigators: John Byrd, William Waldron, Andrzej Wolski

Project Description: An unprecedented level of beam quality is required for the next generation of high energy colliders and light sources. Techniques for manipulating these beams must be developed for these beams in order to achieve new performance standards in future machines. This LDRD has made significant progress in two critical aspects of high-stability beam handling in electron storage rings: broadband feedback systems for controlling instabilities and fast kickers for manipulating individual bunches in the presence of many other bunches without disturbing them. Future accelerators will operate in regimes of intensity and brightness with unprecedented demands on beam quality that will be extremely challenging for feedback systems. For example, one of the dominant effects will be a two-stream instability where the beam couples to a background cloud ions (for a negative beam) that is generated by the intense beam. The ability to control the trajectory of individual bunches in a train of closely spaced bunches in an electron beam, while not affecting the motion of adjacent bunches will open new possibilities for the design and use of future accelerators. Present technologies are not capable of providing significant deflections on individual bunches without significantly larger spacing between bunches than is generally desired. Improving our capabilities for manipulating beams bunch-by-bunch will require significant innovation in a number of areas.

\section{Accomplishments:}

We have developed a system for a precision bunch-by-bunch transient recording system which is presently installed at the Advanced Light Source for testing. This system allows us to record the motion of each electron bunch at a 10 micron resolution, enabling studies of the growth rate of instabilities and the damping rate of the broadband feedback system. An example of the transient response of the kicked beam is shown in the figure to the right. Thjs capability has enabled us to perform initial studies of two-stream instabilities for future storage rings. Further studies will continue in 2007.

We have completed electromagnetic modeling of a fast stripline kicker intended for use as a single bunch kicker. This model allows us to study the time response of the kicker to pulses from an external generator and from the beam. Shown in the figure on the right is a simplified version of the stripline model developed using CST Microwave Studio. This modeling will lead to a mechanical design and construction of an actual kicker in 2007 which will be tested in the Accelerator Test Facility in Japan.
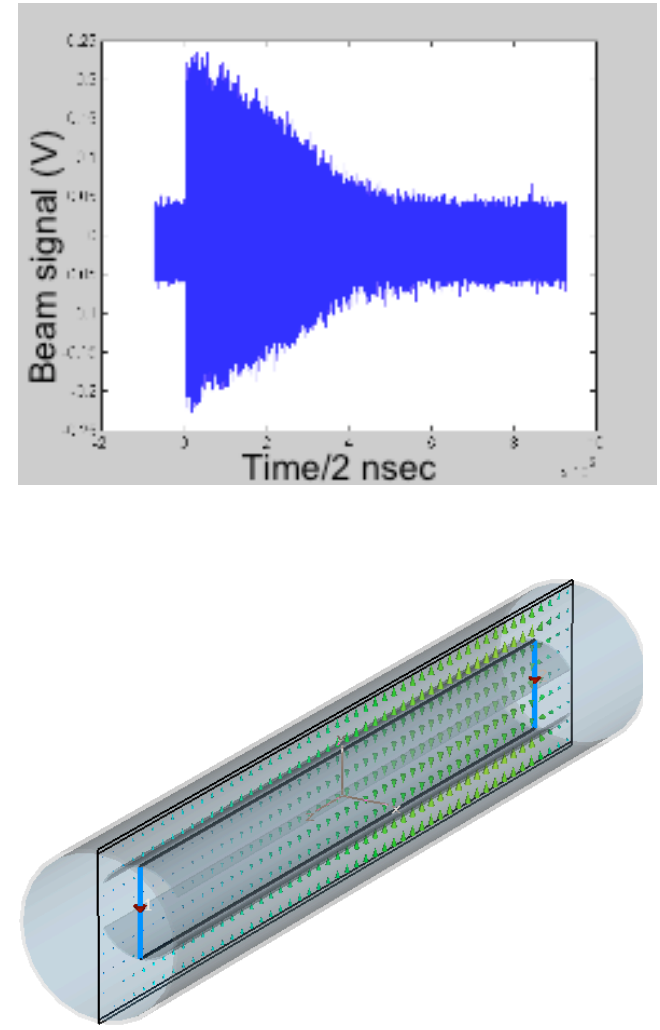
Critical Accelerator Technologies for Advanced Light Sources

Principal Investigator(s): S. Lidia, J. Byrd, J. Corlett, S. DeSantis, R. Duarte, S. Leone, H. Padmore, D. S. Robin, F. Sannibale, C. Steier, R. Schoenlein, S. Virostek, W. Wan, R. Wilcox,

\author{
A. Zholents.
}

Project Description

Critical science and technologies for enhancing the capabilities of the ALS and future generation light sources at LBNL will be developed. Techniques for improved production of intense soft and hard x-ray pulses, including high repetition rate sub-picosecond pulse and increased brightness, will be studied. The areas to be addressed are techniques for production of high-brightness and sub-femtosecond x-rays in a single storage ring lattice, with a variety of operating modes, and injected by a superconducting linac fed by high-power, high-brightness photoinjector systems. In addition, photon production in insertion devices following the linac, and independent of the storage ring, will be studied. Control of the electron bunch properties in the combined accelerator systems will be investigated, to provide optimal x-ray production. Ultra-stable synchronization and timing systems will continued to be developed and demonstrated, to allow precise pump-probe experiments in the ultrafast mode, as well as improving stability in the superconducting linac RF cavities.

\title{
Accomplishments
}

We have performed conceptual and feasibility studies for an upgrade to the ALS aimed at providing sub-picosecond photon pulses to users. Utilizing a quasi-isochronous storage ring lattice, we have demonstrated that an increase in x-ray flux by a factor of $10^{3}$ over the flux generated in the existing slicing source is possible. We have performed exploratory studies on a single superconducting linac, providing up to $\mathrm{GW}$ levels of coherent $\mathrm{x}$-rays down to $1 \mathrm{~nm}$ wavelengths with sub-picosecond pulse lengths. This latter scheme provides the flexibility, stability and performance required in a next generation light source.

We have demonstrated a novel optimization scheme of electron bunch formation and longitudinal distribution to compensate for space charge and wakefield effects in high energy linacs, resulting in a flat current and energy profile of the beam.

To improve timing and synchronization, we have demonstrated independent, simultaneous stabilization of two optical fibers, for comparison in an interferometer. The relative phase delay stability was $\sim 5 \mathrm{fs}$ (peak-to-peak), comparable with one fiber alone. We stabilized a $2 \mathrm{~km}$ length fiber and compared its stability with a $2 \mathrm{~m}$ fiber, also to $\sim 5 \mathrm{fs}$. For stabilizing over long distances, we implemented a frequency stabilization scheme by frequency-locking a $\mathrm{CW}$ laser to a molecular absorption line to one part in $10^{9}$, which is required by the increased length of the interferometer arm. We have synchronized independent fiber lasers, demonstrating 12fs RMS timing stability between two fiber lasers with RF cables linking the laser controls. When the synchronization signal was transmitted over stabilized fiber, the demonstrated stability was 19fs RMS.

We have developed a novel design for a high-brightness, high repetition rate electron beam source, utilizing a low-frequency $(50-100 \mathrm{MHz} \mathrm{CW})$ normal-conducting cavity, to provide beam bunches at rates exceeding $1 \mathrm{MHz}$. Beam dynamics studies demonstrate optimized production of $1 \mathrm{nC}$ electron bunches with sub-micron normalized transverse emittance. 


\section{Enabling High Energy Density Physics at LBNL Principle Investigator(s): Grant Logan, Jonathan Wurtele, Wim Leemans Project Description}

High Energy Density Physics (HEDP) is an emerging field of science to understand the behavior of matter in extreme conditions of temperature and density. HEDP experiments offer us the potential to resolve fundamental questions about plasmas in several regimes important to our understanding of the universe. Our goal is to advance the technological basis of HEDP by developing computational tools and necessary diagnostic capabilities to conduct exciting high energy density plasma experiments using laboratory lasers and accelerators. Our proposal is to a) study new directions in HEDP experiments driven by intense focused particle beams using theory and advanced computation necessary for HEDP, and to b) develop cutting edge short pulse X-ray diagnostics for HEDP scientific exploration. This will be accomplished by two tasks directed at computer codes and laser/X-ray scattering experiments over three years FY05-06-07:

Task 1: Conduct broad surveys of existing experimental techniques used to study equation of state and other properties of high energy density plasmas.

Task 2: Using the LOASIS laser facility and with particle, fluid and hybrid codes, study production of $\mathrm{x}$-ray beams from laser-accelerated electron beams for HEDP target diagnostics. Accomplishments

Task 1 Simulations of particle beam heating of target foils and foams have been studied QEOS and LEOS codes, as well as an EOS code developed recently by R. More (working with our LDRD team) using the Saha equation for ionization and solid state data for excitation energies. We are working with the computing research department at LBL to implement QEOS as part of their hydrodynamics code. This code has adaptive mesh refinement technology and uses their simulation tools package, Chombo. HYDRA simulations were performed for beam-heating of water ice to $0.07 \mathrm{eV}$. Microscopic atomic models for various target materials, including metals and halogens, have been constructed. We used statistical mechanics to construct preliminary models for beam darkening of transparent insulators (glass) and conduction in electronegative plasmas $(\mathrm{Br} 2, \mathrm{I} 2)$ to provide useful temperature markers for beam-heated targets. We have assisted in the scientific organization of the Workshop on Accelerator-Driven Warm- DenseMatter Physics, held in February '06, and participated in two 2006 WDM workshops in Japan. Task 2 We have continued detailed of x-ray radiation from laser-accelerated electron beams. The outcome of this study indicates that two mechanisms can be used to generate bright x-ray beams with $\mathrm{x}$-ray photon energies ranging from a few $\mathrm{keV}$ to multi-MeV. The first method relies on emission of $\mathrm{x}$-ray photons via betatron oscillations that the electrons undergo while being accelerated in plasma channels. This was described theoretically in 2002 by Esarey, Shadwick, Catravas and Leemans with relevance to both laser and beam driven accelerators. We are in the process of studying this radiation on our $100 \mathrm{MeV}$-class accelerator at LOASIS. A two dimensional fiber coupled CCD camera with phosphor screen has been fielded to measure the $\mathrm{x}$ rays. In addition, a collaboration has been successfully initiated between researchers from LOASIS and LBNL's Physics Division on using Si-strip detectors and Si pixel detectors and with the German GSI (Darmstadt) on polarization sensitive X-ray detectors. These experiments allow us to develop the necessary know-how for detecting the radiation and how it depends on the accelerator performance. Two students are currently working on these experiments for masters and Ph.D. dissertation and a postdoctoral researcher will join the effort starting in March 2007. After testing on the $10 \mathrm{TW}$ laser target system, these x-ray diagnostics will be implemented on the GeV electron beam line at LOASIS. 
Low Energy Spread Electron Source

Principal Investigators: Max Zolotorev, Eugene D. Commins, Jim Oneill, Fernando Sannibale, Weishi Wan.

\section{Project Description}

The purpose of the project is to explore a novel approach for a pulsed electron source that has the potential to approach the quantum-limited brightness and lowering the effective source temperature from the current $\sim 100 \mathrm{meV}$ to $\sim 10 \mu \mathrm{eV}$. Such a source can open a wide range of novel applications that utilize angstrom-scale spatial resolution and $\mu \mathrm{eV}$-scale energy resolution. Examples include angstrom-scale resolution electron microscopy, electron holography and investigations of dynamics on the ps time scale using pump-probe techniques. The technology available within the proposed budget will constrain the effective temperature to the meV-range. In this multi-year LDRD we will demonstrate this proof of principle by constructing the source and measuring its temperature to the achievable limit.

In the scheme, an effusive source generates a beam of neutral Cesium atoms. These atoms are then excited to a Rydberg level very close to ionization $\left(\sim 10^{-5} \mathrm{eV}\right)$ in the interaction region (IR) defined by the overlapping of 3 laser beams. Two of the lasers are CW while the third is pulsed with a rep-rate of $10 \mathrm{MHz}$. The parameters are tuned to produce an average of 1 Rydberg excited atom per pulse. After the laser pulse, a delay $(\sim 40 \mathrm{~ns})$ is introduced to allow the electron in the excited atom to travel far from the ion $(\sim 70 \mu \mathrm{m})$ reaching the turning point of its orbit so as to lose practically all of its kinetic energy. At this point, a pulsed voltage is applied to finally ionize the atom and to deliver the electron to the optical system for the final acceleration and for the energy analysis. Last, a second pulsed electric field removes the remaining ion from the IR. In this way the Coulomb interaction effects are minimized and a very high brightness electron beam is obtained.

\section{Accomplishments}

During this second year of LDRD, the source design has been completed, almost all the parts have been fabricated or purchased, and the assembling of the source has started.

A diagnostics system, based on micro-channel plate (MCP) detectors and integrated with the source itself, has been developed. Time of flight measurements of the generated electrons will allow for the source tuning and for the characterization of its performance.

An important novel technique that includes the frequency chirping of the pulsed laser has been conceived. Calculations have shown that with such a scheme, the (already very high) brightness of the source can be further increased for nearly approaching the quantum limit for fermions. The technique will not be used in this exploring project because it requires an additional expensive laser suitable for the frequency chirping, but it could be easily applied to the source in the future.

Full assembling and testing of the source is scheduled to happen in this third year of the project. 


\section{Advanced Light Source Division}

LB04013

Coherent x-ray diffraction imaging (CXDI)

Principle investigators: Malcolm Howells, Anton Barty, Henry Chapman, Chris Jacobsen, Janos Kirz, Stefano Marchesini, John Spence, Uwe Weierstall.

Project description

We report work in the third and final year of a project to develop and demonstrate a new form of high-resolution three-dimensional microscopy (CXDI) using x-ray synchrotron radiation from the Advanced Light Source (ALS). In the first two years the feasibility of the technique was demonstrated for both life- and materials-science samples and excellent performance was achieved in regimes of resolution and sample size not accessible to other forms of microscopy. Even more attractive performances, limited by radiation damage in life science and x-ray source brightness in material science were projected for the future as described in more detail in the reports and publications from those years. Based on these achievements, a modern optimized approach to implementing CXDI at ALS was conceived. The main thrust for FY2006 was therefore scientific and technical studies toward the practical realization of such new approach.

Accomplishments

First studies were to develop the optimum design for the x-ray source and optical system for a CXDI experiment. One outcome of these studies was the recognition that requirements for coherent X-ray scattering and CXDI were quite similar and could be met by sharing a common undulator source.

Some of the characteristics of the technical systems studied under the LDRD are as follows. The x-ray source for the project is an in-air Apple II-type elliptically polarizing undulator. with period $32 \mathrm{~mm}$, length $2 \mathrm{~m}$, vacuum gap $5 \mathrm{~mm}$, magnet gap $7.5 \mathrm{~mm}$, remanent field $1.4 \mathrm{~T}$, and magnetic material Neomax $48 \mathrm{H}$. The in-air design, which has already been implemented at three ALS beamlines, is pushed to higher performance by use of a smaller period and smaller vertical gap. Vacuum gaps of $5 \mathrm{~mm}$ have already been demonstrated at the ALS, and gaps of that size will be used routinely after the top-off upgrade. This source will provide a brightness greater than $10^{19} \mathrm{ph} / \mathrm{sec} / \mathrm{mm}^{2} / \mathrm{mr}^{2} / 0.1 \% \mathrm{BW}$ over the full required energy range of 0.25-3.0 keV.

The CXDI beam line can use a fixed-slit, horizontally-dispersing, spherical-grating monochromator fed by a condenser mirror. This device is designed so that the monochromator slit system isolates a single wave mode while at the same time providing the desired monochromaticity of the beam (about 1500). This latter figure is determined by the resolving power of the imaging process. The fact that a (coherent) x-ray wave mode has very low phase-space area automatically implies a beam of very low angle (large $f$-number) and thus low aberrations generally and essentially unlimited depth of focus. This all allows the technical requirements to be met with a very simple optical system.

A proposed new facility for both coherent x-ray scattering and CXDI experiments, now known as the COSMIC project, was therefore defined and was subsequently chosen to form a Lawrence Berkeley National Laboratory response to the DOE Office of Science Program Announcement "LAB 06-13 Mid-Scale Instrumentation". A full-scale COSMIC proposal was written and submitted to DOE in August 2006. 


\author{
Lensless Imaging of Yeast Cells \\ Principal Investigator: Janos Kirz
}

\begin{abstract}
Project Description
Our motivation for developing diffraction based imaging of non-crystalline biological specimens is based on its unique characteristics, that unlike other forms of imaging, the resolution is not limited by the technology of the fabrication of optical elements such as zone plates. Our goal is to develop the technique, and image frozen hydrated yeast cells (Saccharomyces cerevisiae) in three dimensions at about $15 \mathrm{~nm}$ resolution. The improvement in resolution compared to what has been accomplished (or is likely to be accomplished in the foreseeable future) by other techniques (such as the $\mathrm{XM} 1$ microscope) is expected to provide insight into the life cycle and the interrelation of the organelles within this important model organism. We collaborate on the preparation of the specimens and on the interpretation of the results with some of the noted experts in yeast biology, such as Prof. Aaron Neiman of Stony Brook.

Data collection makes use of the ALS 9.0.1 undulator beamline built by Malcolm Howells. We brought and installed a custom built experimental apparatus, built by the Stony Brook group. This apparatus is permanently mounted on the beamline, and its use is shared by the Livermore/ASU/ALS group. It is designed to accommodate specimens between room temperature and liquid nitrogen temperature. It is capable of collecting diffraction data sets over a wide range of tilt angles under computer control.

Graduate student Enju Lima collected data for her $\mathrm{Ph}$. D. dissertation from frozen hydrated yeast cells, and Xiaojing Huang continued further development of the apparatus.
\end{abstract}

Accomplishments

During the past year we succeeded in collecting complete 2D data sets from frozen hydrated yeast spores, and obtaining a reconstruction. These results formed the $\mathrm{Ph}$. D. thesis of Enju Lima.

We worked with colleagues in Stony Brook, Oregon, Arizona State, Livermore and LBNL to complete technical studies in support of a white paper and then a proposal for a dedicated beamline for coherence based applications. The proposed beamline, called COSMIC, is under review at DOE/BES. 


\title{
Measurement of Molecular Shape using X-ray Scattering
}

\author{
PIs: M. Marcus \& H. Padmore (ALS), P. Adams (PBD), N. Balsara and R. Segalman (MSD, UCB), \\ J. Berger \& J. Kuriyan (PBD,UCB), J. Kornfield (Caltech), E. Kramer (UCSB), W. Bras (ESRF)
}

\section{Project Description}

The goal of this work is to establish a capability at the ALS for the measurement of the shape and assembly of complex molecules and polymers. Applications range from determination of the structure of large protein complexes, to the study of the dynamics of polymer nanostructure assembly. In protein structure for example, it is often difficult or impossible to crystallize very large complexes. X-ray scattering from proteins in solution can be used to measure a low resolution molecular envelope into which the atomic structures of sub-units can be docked, or to elucidate differences between a crystalline structure and a structure in solution. In the polymer nanostructure arena, many methods have been developed for synthesis of the basic building blocks, but engineering the chemistry of the self assembly of these units into useful structures requires knowledge of the assembly pathways. X-ray scattering allows one to measure these processes in detail.

\section{Accomplishments}

The project is just entering its $2^{\text {nd }}$ LDRD year. The first year has focused on conversion of an existing x-ray beamline at ALS to x-ray scattering. This has required that we put a multilayer monochromator in the existing beamline, add sets of slits, and completely rework the equipment in the end station hutch. The endstation will be equipped with a wide angle $\mathrm{x}$-ray detector, a small angle $\mathrm{x}$-ray detector and $4 \mathrm{~m}$ flight tube, as well as a flexible sample environment. The sample environment will accommodate solution scattering cells for proteins, and a sophisticated environment for polymer samples. The latter will include a grazing incidence geometry for studying polymer surfaces, a system for pulling polymer films, an oven for heating films and optical diagnostic equipment. The detectors have been redeployed from our protein crystallography colleagues at the ALS, as they upgrade to larger systems. The detectors have been tested, and a software environment for data collection and analysis has been designed.

We now expect to have $1^{\text {st }}$ light into the new system in Jan. 2007. We also have started to work on definitions of the first phase of the experimental program with our co-PIs in UCB, UCSB and Caltech. The a staff scientist (Marcus), postdoc (Hexemer) and student (Kirian) have been to the European Synchrotron Radiation Facility (ESRF) in Grenoble, to conduct SAXSWAXS experiments as part of the process to learn the details of the techniques. This was in collaboration with our ESRF co-PI, Wim Bras. In addition to the above, we have also started to work on detector issues related to msec time resolved WAXS. We are developing a small prototype detector based on a silicon strip detector and existing CMOS counting electronics produced for an LBNL HEP program and expect to be testing this shortly. It should provide revolutionary performance for WAXS with msec framing rates. 


\section{Left-Handed Nanoscale Meta-Materials: Towards the Optical Domain}

Principal Investigators: Michael C. Martin, Zhao Hao, Eric Anderson and Alex Liddle

\section{Project Description}

Artificial meta-materials can be made that have a negative index of refraction, which allows a homogeneous flat slab of the material to behave as a "perfect" lens, possibly even creating sub-diffraction limited focusing. The meta-materials consist of split-ring resonators which provide a negative $\mu$, and metal strips which provide a negative $\varepsilon$. The goal of this LDRD is to fabricate and measure left-handed nano-scale meta-materials taking advantage of nanofabrication expertise within the Center for X-Ray Optics.

\section{Accomplishments}

We received LDRD funding in FY2005 and FY2006 with the goal of creating fully lefthanded nano-scale metamaterials at infrared frequencies. We have developed detailed electric and magnetic response simulation capabilities, micro- and nano-fabrication techniques to place these test structures on thin $\mathrm{SiN}$ windows, and careful optical measurements of the resultant meta-materials using the ALS infrared beamlines 1.4.2, 1.4.3 and 1.4.4. We overcame a technical hurdle for the micro- and nano-fabrication where a thin plating base of gold can now be removed without affecting the lithographically defined structures. Examples of split ring resonantor structures having nanometer dimensions are shown in Figure 1, right.

These test structures have been measured for their optical properties as a

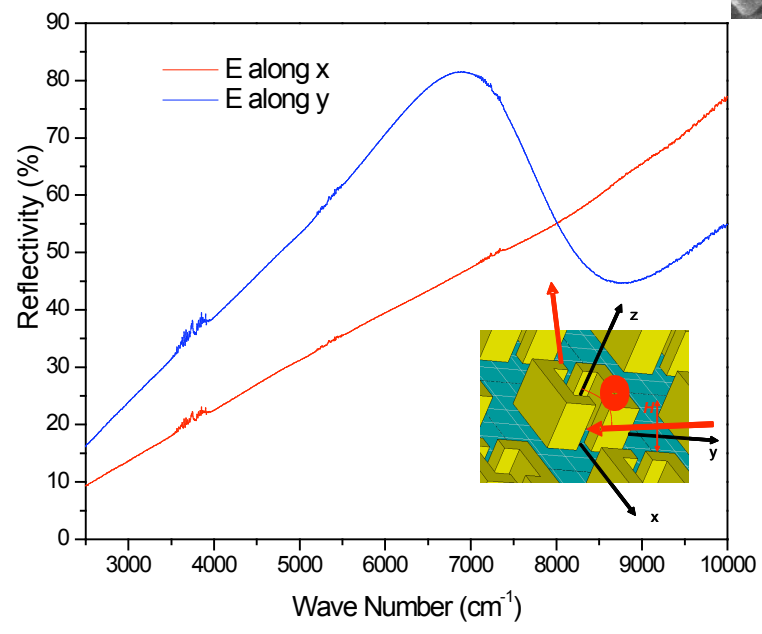

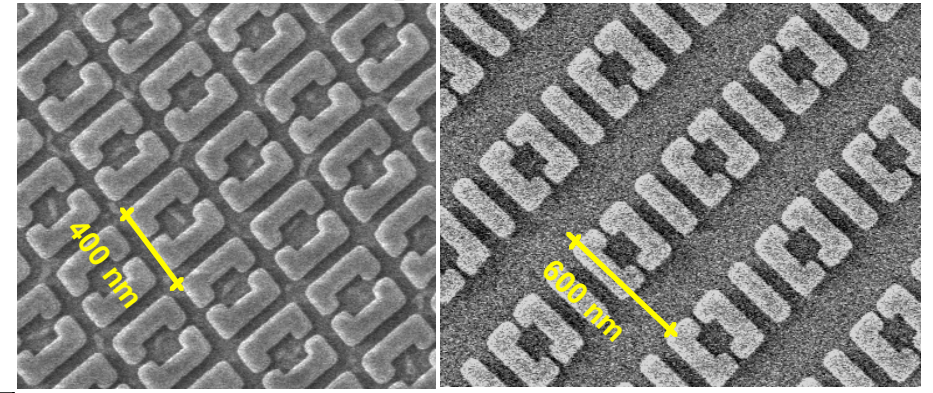

function of polarization and angle of incidence. This allows us to fully characterize the electric and magnetic responses of these materials. An example of a strong polarization-dependent resonance at near-infrared frequencies is shown in Figure 2, left.

We continued with a systematic investigation into split ring resonator geometries and their resultant optical properties. This study will help us to optimize the most favorable geometries for extending these novel resonant structures further towards visible light frequencies, and also will illuminate where

materials properties may become limiting factors.

Our results to date have been presented during FY06 at the 2006 APS March Meeting, an invited talk at the International Workshop on Electromagnetic Metamaterials: Phenomenology and Applications, as a keynote address in the IRMMW-THz 2006 conference in China, and will again be presented at the upcoming APS March Meeting in 2007. 


\title{
NanoARPES:A New Detector for nm-scale Electronic Structure Measurements
}

\author{
Eli Rotenberg* and Aaron Bostwick (LBNL, ALS) \\ *Principal Investigator
}

\section{Project description}

The goal of the nanoARPES project is the development of a new probe for electronic structure measurements at the $25 \mathrm{~nm}$ length scale. The uniqueness of this probe arises from its ability to resolve the spectral function and the momenta of the electronic states, fundamental properties that cannot be determined by any other technique than Angle Resolved Photoemission Spectroscopy (ARPES). Determining these properties for nanometer devices will have an impact on the fundamental understanding of correlated systems, quantum phase transitions, as well as practical problems in semiconductor physics, such as in solid-state lighting, quantum computing, and solar power generation. But extension of conventional, state-of-the-art ARPES, in which a $\sim 50 \mu \mathrm{m}$ focused beam of soft $\mathrm{X}$-rays induces photoelectron emission into an angle- and energy-resolving detector, to the nanometer scale requires novel solutions to problems on both the photon focusing and electron detection sides of the problem. In addition, demonstrations are needed to show the feasibility of the probe, and to show that potential obstacles such as space charge effect are not serious.

\section{Progress report (second year of 3-year LDRD)}

In the first year LDRD, we constructed a testbed instrument and began preliminary measurements, designed to prove feasibility and to attract scientific interest. In the present renewal year, we completed the preliminary measurements (see figure 1). This represents the first spatially resolved ARPES $(300 \mathrm{~nm})$, well below the present worldwide state of the art $(\sim 50 \mu \mathrm{m})$. We began design and construction of a second generation instrument, capable of better spatial resolution $(<100 \mathrm{~nm})$ and with real-world capabilities such as purer vacuum, magnetic shielding, and cryogenic sample holders. The second-generation instrument is expected to come into service to the research community in FY07. We began a conceptual design of a third-generation instrument, capable of resolution down to $25 \mathrm{~nm}$. This instrument, together with a new beamline, will be designed in the third and final renewal year, in collaboration with ALS and Engineering divisions.
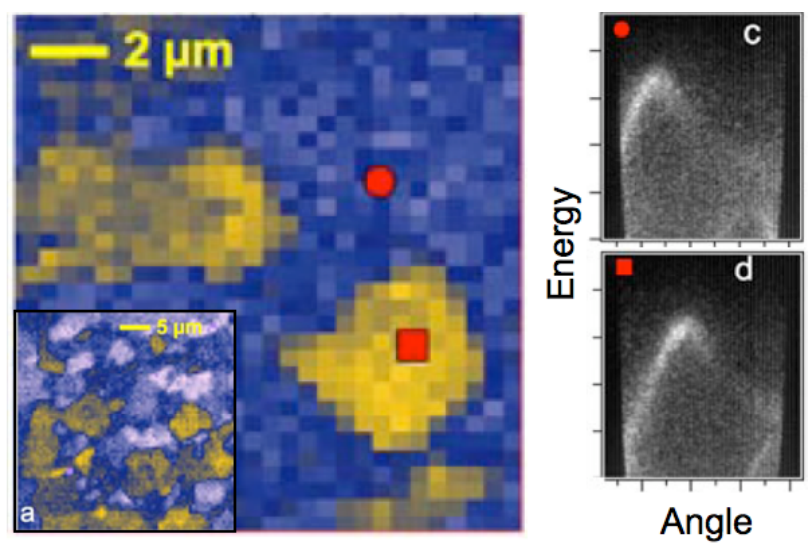

Figure 1. (left) individual crystalline grains in polycrystalline (HOPG-type) graphite. The inset shows a large scale view $(40 \times 40 \mu \mathrm{m})$ bar. This is the first submicron image with angle-resolved valence band contrast. (right). The fully angle-resolved spectra from two different grains. The acquisition time was $10 \mathrm{sec}$ each.

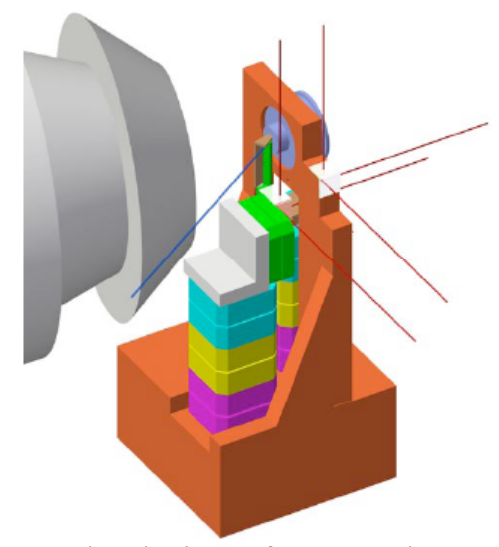

Figure 2. The design of a second-generation cryogenic scanning stage capable of $<100$ $\mathrm{nm}$ spatial resolution, which is currently under assembly. The electron detector is the cylinder to the left. The scanning stages are mounted onto the cryogenic stage at the right. 
Ultrafast Magnetization Dynamics

PIs: Andreas Scholl, Anthony T. Young, Jun Feng, Joachim Stöhr, Howard A. Padmore

Project Description

This project is designed to develop techniques for the study of ultrafast dynamics in magnetic materials. On its own, this project is at the forefront scientific interest. However, it will also help to fill a large gap in the development of the scientific case for the next generation ultrafast x-ray facilities, in addition to offering a new, very exciting area for early exploitation on the ALS f-sec slicing source. Therefore it should be considered a part of the strategic development of ultrafast science and facilities at LBNL. The goals are to demonstrate that we can measure spin and orbital moments in magnetic systems as a function of time after laser or magnetic field excitation on a p-sec streak camera and to investigate the range of scientific applications possible for such a revolutionary new tool.

\section{Accomplishments}

The project is well on the way towards achieving sub-picosecond temporal resolution using $\mathrm{x}$-rays. A record time resolution of about 300 femtoseconds was achieved using UV radiation. This result is especially remarkable because it shows the averaged multiple shot resolution. In beamline experiments a time resolution of 1-2 picoseconds was typical. Experiments were carried out on several systems: $\mathrm{Ni}, \mathrm{Fe} / \mathrm{Gd}$ (in collaboration with U Bochum and U Stuttgart), FeNiPt (in collaboration with Hitachi), $\mathrm{Co}_{2} \mathrm{MnSi}$ and $\mathrm{CoMnSb}$ (in collaboration with $\mathrm{U}$ Bielefeld). In all cases a full or partial phase transition was achieved using an intense IR pump pulse. The speed of the transition and the transient spin and orbital momentum were recorded using x-rays from ALS beamline 4.0. The transition could be fully resolved in the half metallic Heusler alloys $\mathrm{Co}_{2} \mathrm{MnSi}$ and $\mathrm{CoMnSb}$, for which a remarkably slow demagnetization was observed, compared to the sub-picosecond dynamics of a simple metal like Ni. The slow demagnetization of the half-metallic state is probably the result of the suppression of spin-flip scattering at the Fermi level, due to the lack of empty minority electronic states in the half metal. A slowed phase transition was also observed in Fe/Gd, likely due to the suppressed Gd $4 \mathrm{f}$ orbital momentum and the large Gd spin momentum, which is weakly coupled to its environment via exchange with the Gd 5d states. A fast demagnetization was observed in the recording material FeNiPt, which has a density of states similar to a simple transition metal ferromagnet like Ni. Analysis of the spin and orbital moment dynamics of Fe in Fe/Gd showed that ultrafast phase transitions, which had been observed before using magneto-optics, are indeed demagnetization transitions with a true transfer of angular momentum from the electron spin via the orbital momentum to the lattice. A fast spin and orbital momentum thermalization within our temporal resolution was observed.

Recently the laser facility was moved to a new, permanent location at the ALS at beamline 11.0. We anticipate continuing research on ultrafast dynamics, supported by a recently submitted DOE grant proposal, and expect to establish a user program, seeded by our outside collaborations. Improvement of streak camera technology, fast readout CCD cameras, and new sources providing femtosecond $\mathrm{x}$-ray pulses will be greatly beneficial to the program. 


\section{Chemical Sciences Division}

LB04038

Gas phase studies of the building blocks of life

Principal Investigator: Musahid Ahmed

\section{Project Description}

The purpose of this project is to study the fundamental properties of molecules that are the building blocks of life. Dipole moments, ionization energies, electron affinities, proton affinities, molecular polarizabilities and electronic structure all contribute to the photochemistry, radiationless processes and reactivity necessary for biological functions. Fragile biomolecules are generated in the gas phase and time-of-flight mass spectrometry with tunable vacuum ultraviolet (VUV) light is used to measure these properties. Single photon ionization of these building blocks coupled to soft desorption techniques allow for generation of fragment free mass spectrum for large biomolecules. This opens up windows of opportunity in performing mass spectrometric imaging in real biological systems.

\section{Accomplishments}

We extended our novel bio-nanoparticle thermal vaporization technique to launch a number of fragile biomolecules into the gas phase. The general strategy is to synthesize dry nanoparticles comprised of biomolecules that are then thermally vaporized in high vacuum and "softly" ionized with tunable VUV synchrotron radiation producing nearly fragmentation-free mass spectra. To first demonstrate the general utility of this approach and to illustrate how internal energy affects single photon ionization mass spectra, we examined the amino acid tryptophan in detail. In addition, the ionization energies of a number of biomolecules were measured; for some, these are the first reported measurements. Erythromycin (733 amu) is the largest molecule that has been detected completely fragment free with this technique thus far. Coupling these measurements to electronic structure calculations being performed under the aegis of this program will allow the unambiguous determination of adiabatic ionization energies and the structure of the resultant cation for these bio-molecules.

Gas phase clusters of water with DNA bases (Guanine (G), Cytosine (C), Adenine (A) and Thymine (T)) were generated via thermal vaporization of the bases and expanding the resultant vapor in a continuous supersonic jet expansion of water seeded in Ar. The resulting clusters were single photon ionized with tunable VUV synchrotron radiation, and analyzed using reflectron mass spectrometry. Photoionization efficiency curves (PIE) were recorded for the DNA bases and the following water (W) clusters - G, GWn (n=1,2,3); CWn (n=1-3); A, AWn (n=1-4); T, TWn (n=1-4). Appearance energies are derived from the onset of these PIE curves and compared to theoretical and experimental results reported in the literature. For some of these systems, these are the first experimental determinations. During the course of the work on DNA hydration, measurements were also performed to generate appearance energies for water clusters up to $n=60$.

New experiments were developed to allow VUV post-ionization of molecules laser desorbed and ion sputtered from surfaces. Such experiments provide valuable data necessary to assess the viability of the chemical imaging facility at the Chemical Dynamics Beamline as well as contributing to a detailed molecular level understanding of desorption processes. DNA bases were desorbed intact utilizing a subnanosecond $\mathrm{kHz} 355 \mathrm{~nm}$ laser and detected with high sensitivity utilizing tunable VUV radiation. These studies were also extended to long chain hydrocarbon systems and plume dynamics was examined with an eye to understand the relevant desorption processes. Ion sputtering experiments utilizing a $\mathrm{C}_{60}{ }^{+}$ion gun allowed for detection of intact amino acids on surfaces. Detection sensitivity was augmented by VUV post-ionization of neutral molecules. These experiments paved the way for a successful proposal to be funded by DOE from within the "chemical imaging on the nanoscale" program. 


\section{Oxygen Catalysis for Biomimetic Energy Conversion Chemistry Principal Investigator: Christopher J. Chang}

\section{Project Description}

The overall goal of this project is to study the basic reaction types of renewable energy chemistry pertinent to carbon-neutral energy cycles. We are particularly interested in reactions that underlie energy storage and release at oxygen-based feedstocks by coupled proton and electron transport. Our synthetic designs are guided by principles of natural metalloenzymes that catalyze oxygen activation and evolution reactions in biological energy conversion. We seek to capture the functional essence of these enzymes and extend their chemistry beyond the constraints of the protein environment to provide pure synthetic complexes that control reactivity within the first and/or second coordination sphere. To meet the massive scales required for sustainable energy cycles and minimal environmental impact, we are devising new catalytic systems that rely on cheap and plentiful first-row transition metals like iron and manganese. We are pursuing two parallel strategies for biomimetic energy catalysis: (i) new primary-sphere environments for controlling multielectron redox chemistry at first-row transition metal centers, and (ii) attaching second-sphere hydrogen-bond pendants to enhance reactivity at these primary synthetic active sites.

\section{Accomplishments}

A number of new pyrrolide ligands have been synthesized to obtain a series of primarysphere iron and manganese complexes in trigonal bipyramidal and tetrahedral geometries. Preliminary screening reveals that some of these systems are capable of mediating oxygen atom transfer to organic substrates via nitrous oxide or iodosylarene oxidants. Evidence for involvement of high-valent iron-oxo and isoelectronic iron-imido species has been obtained. We are extending this work to activations of hydrogen peroxide or dioxygen for olefin epoxidation and aryl $\mathrm{C}-\mathrm{H}$ oxidation reactions.

In parallel, we have explored the effects of second-sphere hydrogen bonding on smallmolecule reactivity at iron and manganese synthetic active sites. Basic pendants afford added stability to high-valent iron(IV)-oxo species, and influence their selectivity for oxo transfer to phosphine or sulfide substrates. Current studies are aimed at adjusting the orientation between second-sphere pendants and first-sphere metal centers to tune reactivity, and extending oxidation reactivity to more challenging substrates, with the ultimate goal of oxidizing hydroxide/water in these cycles. 


\section{Electron Electric Dipole Moment Experiment in a Cold Atom Fountain Principal Investigator: Harvey Gould}

\section{Project Description:}

The long term goal of this project is to discover or rule out a CP-violating electron electric dipole moment (EDM) as small as $2 \times 10^{-50} \mathrm{C}-\mathrm{m}\left(1.2 \times 10^{-30} \mathrm{e}-\mathrm{cm}\right)$. The observation of a permanent EDM would be proof of new physics not included in the Standard Model and is anticipated by a wide range of extensions to the Standard Model. A new cold Cs atom fountain EDM experiment will include new techniques to suppress systematic effects such as electric field quantization.

Accomplishments:

Overall design of the experiment was completed, a budget and time table was prepared, and a proposal submitted to DOE. Development work on a metal-free interaction region with electrically conducting glass electrodes progressed. (Magnetic Johnson noise from metals is a potential source of noise in the experiment.) A formulation of glass with sufficiently low volume resistivity at room temperature to be used for electric field plates, has been identified. 


\section{Time-Resolved X-Ray Absorption Spectroscopy of Metalloporphyrins \\ Principal Investigator(s): Stephen R. Leone}

\section{Project Description}

This project is focused on the development of ultrafast X-ray transient absorption methods using table-top laser systems, with application to the direct probing of short-lived metal-centered excited states in metalloporphyrin and metal carbonyls. In these experiments, photoexcitation with femtosecond pulses in the ultraviolet or near-infrared results in the formation of electronically excited states in the molecules. Ultrashort x-ray pulses will then probe the subsequent time-evolution of these excited states along the relaxation pathway to the ground state. With this technique, we expect to observe processes occurring on timescales of tens of femtoseconds, thereby allowing x-ray probing of these ultrafast processes with unprecedented time resolution.

A table-top amplified Ti:sapphire femtosecond laser system will be used to produce highorder harmonics in a capillary waveguide filled with a rare gas. The harmonic soft x-rays will be passed through the sample together with the optical pump beam. The pump pulse triggers a photoprocess and the high-order harmonics will probe the changes in the transient absorption of the metal center. The harmonic output is then spectrally dispersed with a grating after the sample to preserve the best possible time resolution. The dispersed light is detected in differential transient absorption on a soft x-ray CCD camera to obtain a spectrum of the metal center absorption as a function of time during the metal-ligand charge transfer or dissociation.

\section{Accomplishments}

Initial funding from a FY2005 LDRD enabled the vacuum chambers and optical layout to be designed and assembled. Soft x-ray radiation in the $30-72 \mathrm{eV}$ range is produced via high-order harmonic generation, in which the 2-mJ, 40-fs output from an amplified Ti:sapphire laser system is focused into the entrance of a 150- $\mu \mathrm{m}$-diameter capillary filled with either Ar or Ne.

Funding from a FY2006 LDRD enabled us to demonstrate that high harmonics can be used in both static and femtosecond time-resolved atomic photoabsorption measurements. The nonresonant absorption spectrum of $\mathrm{Xe}$ in the $40-70 \mathrm{eV}$ range is obtained from changes in the peaks of the individual harmonics upon introducing the gas sample. Furthermore, resolving transmission changes within a single harmonic reveals the narrow, resonant transitions originating from the $4 d$ core level. The measured absorption maximum and cross-section for the $4 d^{9}\left({ }^{2} D_{5 / 2}\right) 5 s^{2} 5 p^{6} 6 p \leftarrow{ }^{1} S_{0}$ transition were found to be $65.1 \mathrm{eV}$ (lit. $65.1 \mathrm{eV}$ ) and $13.3 \pm 2.9 \mathrm{Mb}$ (lit. 13.6 Mb), respectively, in good agreement with literature values. Similar measurements on He have also led to the observation of the ${ }^{1} P^{\mathrm{o}}(n=2, N=2) \leftarrow{ }^{1} S^{\mathrm{e}}$ double excitation resonance at $60.2 \mathrm{eV}$.

Time-resolved measurements were carried out to investigate optical field ionization dynamics of Xe with an intense femtosecond laser pulse. Probing the resultant $\mathrm{Xe}^{+2} D_{5 / 2} \leftarrow{ }^{2} P_{3 / 2}$ transition at $55.4 \mathrm{eV}$ with the soft x-rays has enabled the direct observation of hole orbital alignment on ultrashort timescales.

In addition to spectroscopic measurements, an effort was also undertaken to characterize the soft x-ray beam profile. Under optimal conditions, beam waists as small as $17 \mu \mathrm{m}$ and $24 \mu \mathrm{m}$ at the sample focus were obtained for the integrated $\mathrm{Ar}$ and $\mathrm{Ne}$ harmonics, respectively. From the measured far-field beam divergences, the beam quality factor $\left(M^{2}\right)$ was estimated to be $\sim 2$ with either $\mathrm{Ar}$ or $\mathrm{Ne}$ as the harmonic generation medium. This $M^{2}$ value compares favorably with those reported in the literature. 
Spectroscopy and Dynamics of Pure and Doped Helium Nanodroplets

Principal Investigator: Daniel M. Neumark

\section{Project Description:}

This project is aimed at understanding the fundamentals of photoionization and photo detachment of helium nanodroplets. Experiments on neutral droplets doped with an atomic or molecular chromophore will probe the fragmentation processes that occur when the chromophore is ionized, the dynamics of electron ejection from the droplet, and effect of the droplet atoms on the photoelectron spectrum of the dopant. Experiments on droplets photodetachment and photoionization as well as determine whether the anion is solvated on the surface or interior of the droplet.

Photoionization and photoelectron spectroscopy experiments on the neutral droplets will be carried out on the Chemical Dynamics Beamline at the Advanced Light Source, using tunable VUV synchrotron radiation to ionize the droplets. Further experiments employing resonant multiphoton ionization will be carried out using pulsed lasers. Negatively charged droplets will be generated by crossing a doped droplet beam with a low energy beam that will result in either dissociative or non-dissociative attachment to the chromospore.

Accomplishments:

During the past year we have carried out photoionization and photoelectron spectroscopy experiments on $\mathrm{He}$ droplets doped with $\mathrm{SF}_{6}$, rare gas atoms, and aniline. Three types of experiments were performed: photoionization mass spectroscopy, in which the photon mass spectrum is measured using a newly implemented time-of-flight mass spectrometer configuration on the beamline, photoion imaging, in which the photoion velocity distribution is determined for ions formed in droplets, and photoelectron imaging, in which the effect of the droplet on photoelectron energy and angular distributions is determined. The key finding of our experiments has been that in doped droplet experiments at the ALS, photoelectrons are formed by indirect photoionization of the dopant, via excitation or ionization of the surrounding He atoms followed by dopant ionization via a Penning process or charge transfer. In contrast, resonant two-photon ionization directly ionizes the dopant. The two processes apparently lead to opposite shifts in the photoelectron spectrum relative to the gas phase atom or molecule; the photoelectrons are slower for indirect ionization and faster for direct ionization. However, in this project we have looked only at $\mathrm{SF}_{6}$ and rare gas atoms at the ALS, and aniline in our laser lab, future follow-on work will perform indirect photoionization experiments on droplets doped with aniline and compare those results with the direct R2PI experiments. 
Properties of New Ionic Liquids for Electrochemical Applications and for Extraction of HeavyMetal Cations from Wastewaters.

Principal Investigator(s): John Prausnitz

\section{Project Description}

Ionic liquids are promising new solvents for a variety of possible applications in chemical technology and in electrochemistry. The goal of this project is to provide new experimental results toward increasing the as-yet small data base. First, the project aims to synthesize new ionic liquids. Second, it aims to obtain experimental data for both new and conventional ionic liquids; measurements include densities, melting and glass-transition temperatures, decomposition temperatures, viscosities, equilibria with water and electric conductivities. Two possible applications are of particular interest: use of selected ionic liquids to extract specific cations from wastewaters and use of selected ionic liquids for lithium batteries.

Accomplishments

Twenty five new ionic liquids have been synthesized to determine how various large cations affect physical properties. These new cations include several derivatives of pyridinium, piperidinium and pyrrolidinium. Selected physical properties of new and conventional ionic liquids were obtained; these properties include melting temperature, glass-transition temperature, decomposition temperature, density, viscosity over a wide temperature range and, of particular interest to this project, mutual solubilities of water and ionic liquid.

Several of the new ionic liquids show solubilities in water much lower than those of conventional ionic liquids; such hydrophobic liquids, therefore, may be useful for extraction of cations from wastewaters. Toward that end, we measured distribution coefficients for a variety of dilute aqueous cations between an ionic liquid and water. While most ions are not easily extracted using any of our ionic liquids, copper and mercury are totally and selectively extracted using a task-specific liquid, where a disulfide is attached to an octylpyridinium cation.

For possible battery applications, conductivities were measured for neat ionic liquids and for solutions of lithium salts in ionic liquids. Chemical reactions at the electrode-ionic liquid electrolyte interface were studied using interfacial impedance measurements. Lithium-lithium and lithium-carbon cells were studied for an open circuit and for a charged system. Some of the new ionic liquids exhibit superior electro-chemical properties when compared to those of conventional ionic liquids.

Studies have been initiated to determine the toxicities of new and conventional ionic liquids. Mammalian breast cancer cells were exposed to a series of dilute aqueous solutions of ionic liquids for 24 hours. An enzymatic assay determines the number of live cells. Preliminary results indicate that toxicity rises with increasing length of the alkyl ring attached to the imidazolium or pyridinium or piperidinium or pyrrolidinium cation. 


\section{Advanced Computational Methods for Photon-Molecule Collision Processes Principal Investigators: T. N. Rescigno and C. W. McCurdy}

\section{Project Description}

Sophisticated calculations of collisional ionization processes involving two electrons in the continuum have so far been limited to light atomic targets. For analogous processes involving simple molecular targets, no first-principles calculations have appeared to date. The purpose of this project is to develop advanced computational methods for studying certain photon-molecule collision processes that are currently beyond the grasp of first principles methods. We will develop grid-based ab initio approaches, based on the method of exterior complex scaling (ECS), for studying double photoionization (one photon in, two electrons out) of small molecules. These theoretical methods will assist in the interpretation of current experiments at the ALS on double photoionization of $\mathrm{D}_{2}$ and provide a predictive tool for motivating new experiments.

\section{Accomplishments}

Our principal accomplishment during the past year was the completion of an essentially exact, first-principles study of the photo-double ionization of aligned $\mathrm{H}_{2}$, the first such calculation ever carried out for a molecular target. The initial results of this study were published in Science and gave conclusive evidence that the observed patterns of photoejection, which depend both on bond distance and orientation, are a clear signature of changes in molecular electron correlation. We subsequently developed an independent check of these initial results using finite elements and the discrete variable representation and succeeded in obtaining fully converged results for the triple differential cross sections (TDCS). Using these results in an analysis of the COLTRIMS experiments, including averages over finite ranges of acceptance angles and energy sharings, as in the experiments, we were able to uncover certain errors in the analysis of the experimental data. These have since been corrected and there is now excellent agreement between theory and experiment. The results have been published in Phys. Rev. A.

We also examined the origin of the experimentally observed variation of the differential cross sections with internuclear distance and showed that they arise from pronounced differences in the R-dependence of the parallel and perpendicular components of the ionization amplitude. We were also able to use the calculations as a predictive tool to find other geometries and energy sharings where the R-dependence of the differential cross sections would be more prominently revealed than in current experiments. Finally, we showed that R-dependences should be readily observable in the asymmetry parameter for photo-double ionization, even in experimental measurements that are not differential in the energy sharings between ejected photo-electrons. Our results have been submitted for publication to Physical Review Letters.

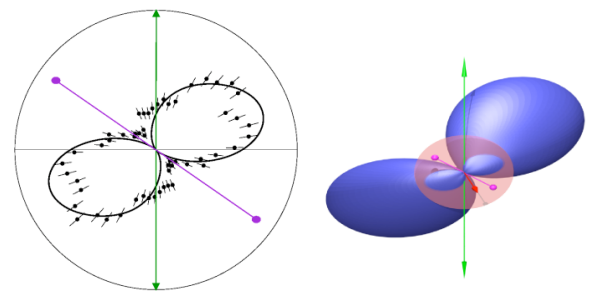

TDCS for double ionization of $\mathrm{H}_{2}$ at $75 \mathrm{eV}$ photon energy and equal energy sharing between the two electrons. Molecule is aligned at 55 degrees to the polarization and the fixed electron is perpendicular to the page. Left: comparison with ALS exp't. Right: full 3D TDCS. 


\section{A New Approach for the Catalytic Conversion of Methane and Other Inert Hydrocarbons Principal Investigator(s): T. Don Tilley}

\section{Project Description}

The selective, catalytic functionalization of saturated hydrocarbons represents one of the most important challenges in chemical research. While some progress has been made, there are very few processes which allow conversion of the cheapest and most abundant hydrocarbon, methane. Intense interest in this topic has led to many important advances including the discovery of several mechanisms by which transition metal species react with unactivated $\mathrm{C}-\mathrm{H}$ bonds. While these studies have revealed a number of interesting stoichiometric transformations, there have been significantly fewer reports describing selective conversions of alkanes via homogeneous catalysis. Methane is a particularly attractive substrate for such conversions since it is cheap and readily available, and represents a potentially useful reagent for the incorporation of methyl groups into molecular structures.

For some years, we have been studying $\sigma$-bond metathesis (the concerted exchange of substituents between two $\sigma$-bonds) as a fundamental process for bond activations, in the context of metal-mediated polymerization chemistry. Studies on the interactions of silanes with $\mathrm{d}^{0}$ metal complexes have revealed several pathways for the activation of $\mathrm{Si}-\mathrm{H}$ and $\mathrm{Si}-\mathrm{C}$ bonds via $\sigma$-bond metathesis. This rich reaction chemistry suggested that similar activation steps might be used in catalytic hydrocarbon functionalizations, given appropriately active and selective catalysts. In preliminary tests of this concept, we have observed the first catalytic processes that allow conversion of methane to other products via $\sigma$-bond metathesis with $\mathrm{Cp}_{2}{ }_{2} \mathrm{ScMe}\left(\mathrm{Cp}^{*}=\mathrm{C}_{5} \mathrm{Me}_{5}\right)$ as catalyst. These observations raise the exciting possibility for development of homogeneous catalytic processes that utilize methane as a cheap and convenient methylating reagent. We therefore plan to pursue this possibility in the development of new catalytic conversions for methane and other inert hydrocarbons.

\section{Accomplishments}

Efforts in this program have centered on exploration of systems related to the scandium one described above, to learn as much as possible concerning the influence of ancillary ligands, reaction conditions, and substrate variations. It is clear that a soft, strongly donating ligand (e.g., $\mathrm{Cp}^{*}$ ) supports $\sigma$-bond metathesis reactivity, and variations in the structure of such ligands should reveal factors that influence reactivity in this chemistry. Since electron-rich, anionic $\pi$-systems are generally good candidates, we chose to target the synthesis and development of the permethylfluorenyl ligand $\left(\mathrm{C}_{13} \mathrm{Me}_{9}{ }^{-}\right.$, Flu* $)$which, due to its size and electronic configuration, has the potential for promoting $\sigma$-bond metathesis reactivity and selectivity in bond activations. The development of a synthetic route to octa- and nona-methylfluorene was accomplished and these compounds were used to prepare mixed-ligand ruthenocene complexes. Structural studies demonstrated that Flu* is $\eta^{5}$-coordinated to the metal center, and initial electrochemical studies of the ruthenium compounds revealed that Flu* is more electron-donating than the Cp* ligand.

We are currently investigating the coordination chemistry of the Flu* ligand, with group 3 , lanthanide and early transition metals. We have prepared a family of Flu* containing zirconium and hafnium compounds and are investigating their reactive properties. Reduction of mixed-ligand zirconocenes in the presence of carbon monoxide gave a series of zirconocene dicarbonyl compounds and a spectroscopic study demonstrated the very electron-donating nature of the highly methylated fluorenyl ligands. Several Flu* supported group 4 compounds containing alkyl or hydride groups were prepared, and showed that the Flu* ligand can change or adapt its coordination mode to help stabilize the reactive metal species. Specifically, attempts to prepare a zirconium dihydride derivative led to the isolation of a complex derived from a hydride transfer from the metal to the Flu* ligand, causing a transformation in its coordination mode to $\eta^{3}: \eta^{5}-\mathrm{C}_{13} \mathrm{Me}{ }_{9} \mathrm{H}$. Investigations of this "masked" dihydride species, $\left[\mathrm{Cp}(\mathrm{SiMe})_{2}\right]\left[\eta^{3}: \eta^{5}-\right.$ $\left.\mathrm{C}_{13} \mathrm{Me}_{9} \mathrm{H}\right] \mathrm{ZrH}$, showed that it can participate in a wide variety of reactions such as hydrogenation, olefin dimerization, and $\mathrm{C}-\mathrm{H}$ activation of pyridine derivatives. 


\author{
Versatile Mini-Scanning Transmission X-ray Microscope (mSTXM) \\ Principal Investigator(s): Tolek Tyliszczak and David K. Shuh
}

\title{
Project Description
}

The objective of this project is to develop a new Scanning Transmission X-ray Microscope (STXM) for the Advanced Light Source (ALS). There is a timely opportunity to design and implement a revolutionary, compact, stand-alone, prototype STXM instrument that will provide additional STXM capacity for scientific investigations utilizing existing beamlines at synchrotron radiation sources such as the ALS without the high opportunity cost. The research team that designed and built the ALS STXMs will develop a compact STXM microscope which can be fabricated at a lower cost than existing STXMs, while at the same time providing the experimental versatility with nearly all of the current STXM performance characteristics. The new microscope, the mini-STXM (mSTXM), is envisioned as a portable end station which could be attached to several existing ALS beamlines for experiments and will also serve as a developmental test platform for future STXM innovations at the ALS.

There are three major technical design challenges which have to be overcome in an innovative and economical manner to successfully realize the portable mSTXM instrument. Very low relative vibrations, precise and fast sample scanning and efficient and time resolved photon detection are those challenges. The approach to meet these technical requirements and the overall goals for the mSTXM, will be the universal utilization of compact, state-of-the-art piezoelectric scanning/positioning stages. The STXM research team responsible for the construction of the two existing ALS STXMs will design, fabricate, and implement the mSTXM over a two-year period.

\section{Accomplishments}

One fundamental part of the initial mSTXM conceptual design was to employ a long range piezo flexure as the sample scanning stage. Intensive experimental studies with a demonstration nanocube unit showed that this piezoelectric stage had too much mechanical noise and therefore can not be used as a basis for the mSTXM. An extensive search for an alternative solution led us to re-examine the entire overall conceptual design. This has resulted in a new, updated design of an even more compact mSTXM, the mSTXMII, that has performance specifications akin to existing large STXMs but comes with an accompanying increase in the estimated per unit fabrication cost. The total overall cost of the first mSTXMII unit still remains less than originally proposed.

The new mSTXMII design will use ultra-high vacuum (UHV) compatible linear positioning and scanning stages obtained from a commercial manufacturer. These stages are commonly used in this manufacturer's atomic force microscopes and according to the specifications, should provide mechanical noise in the range of few nm. This will ensure that the mSTXMII resolution will be limited by the zone plate even when the best foreseeable zone plates become available. Furthermore, the new mSTXMII design eliminates the energy range limitation inherent in the original mSTXM design by using a linear positioning zone plate stage and including a laser interferometer. The use of commercial stages permits the incorporation of these two features and although they add to the cost, they contribute extremely desirable additional functionality that could not be included in the original mSTXM design based on the nanocube stage. Thus, the mSTXMII will have most of the functionalities of existing high performance STXMs and will have some new capabilities.

The full 3D conceptual model of the mSTXMII has been completed and some of the critical components have been procured to enable further testing. The initial mechanical design has been completed, fabrication drawings produced, and the large number of custom mechanical assemblies required for the prototype manufactured on schedule. 


\section{Computing Sciences}

LB05008

Cryptographic Foundations for New Generation Distributed Systems

Principal Investigator: Olivier Chevassut, Abdelilah Essiari

\section{Project Description}

New generation distributed systems (e.g., peer-to-peer and Grid middleware) are networking technologies that enable complex interactions among computational and data resources. These distributed communication middleware employ complex message exchange models (e.g., one-way, asynchronous, bilateral, multi-node paths) that cannot be supported by existing security solutions. If these distributed systems are to be successful and widely deployed in production computing environments the systems need to be enhanced with the adequate cryptographic mechanisms. While the usual security notions are still valid, the message exchange models require new cryptographic foundations.

The present project lays down the security foundations to design and develop an essential component of next generation distributed systems. The project first produces provably-secure cryptographic algorithms such as (one-time) password-based authentication and key exchange for two or more parties. Next, it implements a prototype that will support the complex message exchange models needed by next generation distributed systems. Finally, it makes sure that these algorithms retain their "provablesecure" features in practice by specifying implementation guidelines.

\section{Accomplishments}

The main accomplishments of this project have been new provably-secure cryptographic algorithms for next generation distributed systems. The publications describe cryptographic methods for authentication methods--for two or more partiesthat will play a role in securing next generations distributed architectures.

First, we have analyzed the key derivation process by which an agreed upon large random number is used to derive keys to encrypt and authenticate data. Practitioners and standardization bodies have usually used the random oracle model to get keying material from a Diffie-Hellman key exchange; however, formal analysis require randomness extractors to formally extract the entropy of the random master secret into a seed prior to deriving other keys. We have studied such an application and presented very efficient and provable randomness extraction techniques for mathematical groups under the DDH assumption.

We also examined overcoming the shortcomings of existing cryptographic algorithms for authenticated key exchange. Current solutions are either limited by the use of public key infrastructures or by their scalability--requiring a number of rounds linear in the number of group members. To overcome these limitations, we proposed the first provably-secure password-based constant-round group key exchange protocol. Our protocol is very efficient and fully scalable since it only requires four rounds of communication and four multi-exponentiations per user. The new protocol avoids intricate authentication infrastructures by relying on passwords for authentication.

Additional papers provide detailed specifications for the implementation and for the use of our cryptographic algorithms to make sure that the algorithms retain their provable-secure features in practice. 


\section{Analysis for Simulation of Nuclear Energy Systems Principal Investigator: Phillip Colella}

Project Description: Power from fission reactors has the potential for meeting a substantial fraction of US energy needs. A central component of the science and technology base required for a sustainable future for nuclear energy is the development of computer modeling and simulation tools for the analysis of central technical issues in all areas of the nuclear power cycle, such as the fuel cycle, reactor design, and waste disposal. There are a number of areas in computational mathematics and computational science (numerical methods for partial differential equations, computational fluid dynamics) that are central to simulation in these areas, and in which LBNL scientists are world leaders. This project was to engage in early stages of the technical requirements analysis for simulating all phases of the nuclear power cycle. The technical goals consisted of the analysis of three aspects of the simulation process: analysis of models, algorithmic issues, and high-performance computing issues.

Accomplishments: Our analysis identified three technical issues of central importance in end-to-end modeling for nuclear energy, and for which LBNL has unique expertise.

- $\quad$ Fluid dynamics for multicomponent fluids in complex geometries. This problem arises in the simulation of the reactor core, in heat and mass transport in the drift tunnels in which nuclear waste is to be stored, and in the macroscale design of separations systems. LBNL expertise within CRD in this area includes the analysis of low-Mach number limits for multcomponent and multiphase fluid mixtures and the design of high-resolution and adaptive discretization methods using cut-cell representations of the geometry.

- Neutron transport algorithms. The neutron transport is a critical component of the physics in a reactor core, and is represented by an integro-differential equation in sixdimensional phase space (plus time). LBNL within CRD has expertise in the development of $S_{n}$ methods on structured grids with adaptive mesh refinement for such problems, as well as the use of volume-of-fluid methods for representing the jump relations at interfaces between different materials.

- $\quad$ Subsurface flow modeling. The long-time impact of storing nuclear wastes depends on the transport of contaminants through the soil and rock adjacent to the storage site. Typically, these processes are modeled by porous media equations for multiphase mixtures of contaminants and water. LBNL has a considerable expertise in the development of high-resolution methods and adaptive numerical methods for such problems in CRD, as well as a well-established applied modeling effort in ESD in subsurface flow modeling.

In all of these areas, the methods that would be developed by LBNL are ones for which we have considerable experience in developing efficient parallel implementations. Based on that experience, we expect that we would be able to develop applications codes in these areas that would make effective use of high-end computers. 
Software Application Infrastructure For Efficiently Managing Large-Scale Computational Biology Experiments

Principal Investigator(s): David Konerding

\section{Project Description}

The purpose of this project is to enable large-scale computational experiments being carried out by the Brenner laboratory which are designed to increase our understanding of gene regulation and genetic diseases in humans. These experiments will elucidate nonsense-mediated decay, a eukaryotic mRNA transcript surveillance mechanism which ensures that improperly spliced transcripts are degraded before being translated into protein. The Brenner laboratory performed a combination of computational screens and lab experiments showing that NMD plays an important role in several disease-related genes, including Calpain-10 (susceptibility to Type-II diabetes in several populations), Presenilin1 (linked to Alzheimer's disease susceptibility) and lymphocyte associated receptor of death (associated with lymphoid malignancies). The list of diseases associated with NMD is growing rapidly. However, current work only scratches the surface of detecting NMD candidates. We need a computation-based experimental infrastructure (C-BEI) so we can make a number of important methodological improvements to the NMD detection pipeline, significantly extending its sensitivity and specificity. With this improved pipeline, significantly more sophisticated experiments can be carried out.

We will develop the C-BEI to address critical issues associated with scale-up of NMD candidate detection, including: reliable management of long-running jobs, structured data storage, and an easy-to-use experiment description language. Each of these components will be developed with a well-defined service interface and a modular, pluggable implementation. Using modular, pluggable implementations combined with well-defined interfaces allows us the freedom to experiment with alternative solutions.

\section{Accomplishments}

We developed a visual application, ViCE, for creating experiment descriptions. Based on our initial research, we adopted an XML format very similar to that of the Ptolemy, a UC Berkeley application used to model real-time concurrent systems. We also developed the core $\mathrm{C}$-BEI engine for reliable management of long-running jobs. The visual application is coupled to the C-BEI engine using grid- and web-services technology, allowing users to model their experiments, submit them to the engine, then disconnect from the engine. Later, users can connect to the engine and see the progress of their experiments and visualize the results if the experiments have completed. We also developed a component that collects and stores experimental data. We are currently in the process of measuring the performance of our design using carefully constructed sequence comparison validation experiments which model the Brenner lab NMD pipeline to ensure that our components and architecture attain the performance that is required for scale-up of NMD detection. The ViCE and C-BEI software releases are available at http://dsd.lbl.gov/gtg/projects/vice/ and http://dsd.lbl.gov/gtg/projects/CBEI/, respectively. 


\title{
Integrated Microbial Community Genomes Data Management System
}

\author{
Principal Investigators: Victor M. Markowitz, Natalia N. Ivanova, \\ Nikos C. Kyrpides, and Phil Hugenholtz
}

\section{Project Description}

Studies of the collective genomes (also known as metagenomes) of environmental microbial communities (also known as microbiomes) are expected to lead to advances in environmental cleanup, agriculture, industrial processes, and alternative energy production. Similarly, studies of the metagenomes of human microbiomes will provide new insights into variations of microbial populations associated with the human body in health and disease, and will lead to the development of new treatment strategies.

The purpose of this project is to develop metagenome analysis methods and tools in the context of integrated microbial genome and metagenome data. A metagenome analysis toolkit (MGAT) will provide analytical capabilities allowing scientists to study metagenomes together with other available microbial genomes and metagenomes integrated within a metagenome data warehouse. MGAT will help address fundamental biological questions, such as how are functions distributed within a community and how does a community respond in both the near and long term to environmental changes.

\section{Accomplishments}

Our main accomplishment has been the development of experimental versions of MGAT tools as part of the IMG/M metagenome data management and analysis system (img.jgi.doe.gov/m). IMG/M supports exploration and comparative analysis of metagenomes in the context of isolate microbial genomes and other metagenomes. Microbiomes can be examined in IMG/M in terms of available metadata along with various statistics of interest, such as the total number of scaffolds and genes or the number of genes associated with KEGG, COG, or Pfam. IMG/M provides a number comparative analysis tools for examining metagenomes, their component bins and isolate genomes in terms of gene content and functional capabilities. Tools for analysis of gene content include a profile-based selection tool, gene neighborhood analysis tools, and multiple sequence alignment tools; functional capabilities can be analyzed using several occurrence profile and abundance profile tools. Additional tools allow analyzing strainlevel heterogeneity within a species population in metagenome data.

IMG/M has proved valuable in completing the analysis of biological phosphorus removing (EBPR) sludge communities and the gutless worm symbionts, and is currently used for studying the metagenomes of several environmental microbial communities sequenced at JGI, including the hydrogen-producing microbiome colonizing the termite hindgut. The recently published human gut metagenome data set has been included into IMG/M, thus providing a unique opportunity to compare allopatric populations of the same bacterial species inhabiting drastically different environments in terms of their gene content and metabolic capabilities. 


\author{
Enhancing Commodity Scalar Processors with Vector Components \\ for Increased Scientific Productivity \\ Principal Investigators: Leonid Oliker, John Shalf, Katherine Yelick, William T. Kramer
}

\title{
Project Description
}

As we enter the era of billion transistor chips, computer architects face significant challenges in effectively harnessing the large amount of computational potential available in modern CMOS technology. We are increasingly constrained by the comparatively lower rate of improvement for off-chip connections. The increasing gap between processor and memory speeds has become a well-known problem in computer architecture. Furthermore, techniques designed to hide memory latencies, such as out-of-order superscalar instruction processing, speculative execution, multithreading, and stream prefetching engines, may actually increase the memory bandwidth requirements. This so-called "memory wall" is one of the primary reasons many high performance applications run well below the peak arithmetic performance of the underlying machine. In particular, irregularly structured and data-intensive codes exhibit poor temporal locality and receive little benefit from the automatically managed caches of conventional microarchitectures. In addition, a significant fraction of scientific codes are characterized by predictable dataparallelism that could be exploited at compile time with properly structured program semantics; supercalar processors can often exploit this parallelism, but their generality leads to high costs in chip area and power, which in turn limit the degree of parallelism.

The goal of our research is to address these challenges by exploring the opportunities for improved scientific productivity on two fronts. First we examine alternative caching strategies, such as software controlled memory architectures, with the goal of leveraging these techniques to improve scientific productivity. Next we explore the potential of combining vector processing features with commodity processors. In particular, we are identifying the minimum set of hardware features required to see the benefits of vectorization without compromising the costeffectiveness of commodity processors for general purpose processing.

\section{Accomplishments}

Extensive progress has been made in the last year on evaluation of the Cell processor for high performance computing. In addition to modeling the basic dense matrix multiplication and FFT kernels, both the heat equation stencil and a sparse matrix multiplication code were modeled, implemented, and evaluated on a $3.2 \mathrm{GHz}$ Cell blade. Preliminary results were presented in a paper at Computing Frontiers (CF06), and additional data was presented at both the EDGE workshop as well as the Global Signal Processing expo (GSPx). Subsequently, the temporally blocked heat equation was improved and compared against similarly optimized implementations on the Opteron, Power5 and Itanium2. More recently, the central function of a full application (LBMHD3D) was implemented in double precision on the Cell processor. Results demonstrated that where the performance of scalar processors diminish with increasing complexity, Cell, like vector machines, can improve absolute performance.

We are also exploring vector extensions to microprocessor ISA by extending the IBM Mambo simulator to simulate VIVA-2 (or vector extensions) on the Power processor. The ViVA-2 simulator is now verified to be working correctly, both in fast functional mode and cycle-accurate mode. Our implementation includes optimization work that removes the need for memory sync instructions when transferring data between the ViVA-2 buffer and scalar registers. All of the support software -- compilers, linkers, assembler, debugger -- have been modified to run in the simulation environment. Currently, we are exploring the behavior of the striad stanza triad benchmark, to understand potential performance advantages of the VIVA-2 approach and verify that timing accounting is operating correctly. Once this testing is complete, we plan to examine to a broad range of numerical kernels, particularly codes that can take advantage of simultaneously active streams, indexing, and software-managed memory buffers. 


\section{Advanced Computational Tools for Electric Power Systems}

Principal Investigators: Ali Pinar, Bernard Lesieutre, Chao Yang, and Juan Meza

\section{Project Description:}

As society's dependence on engineered networks such as the electric power, communication networks, and the Internet expand, the need for secure and reliable operational standards for these systems becomes vital for the economic, energy and national security of our country. The August 14, 2003 blackout in the Northeast depicted the possible catastrophic consequences of a few broken power lines. This blackout affected 50 million people in the Northeast, its estimated cost in the U.S. was $\$ 4-10$ billion, and it took as many as 4 days to recover.

The need for improved operational and reliability standards for the electric power system is incontestable. Such efforts however, are hindered by the lack of computational tools that can provide better modeling capability and system understanding. We claim that the improved reliability standards for electric power systems will come with advanced computational tools and high performance computing, and LBNL as a renowned institution in optimization and environmental energy research has a great opportunity to step up to be the center of research in computational electric power systems.

\section{Accomplishments:}

We have developed several mathematical formulations and devised algorithms for the vulnerability analysis problem, where we seek a small group of lines, removal of which will cause a significant disturbance to the system. Since these optimization problems are compute intensive, especially for larger problems, we have devised alternative formulations that trade off between the model accuracy and problem complexity. This range of models not only allows us to use the computationally easier models to be used as surrogates for the solution of formulations with more sophisticated models, but also promote wide applicability of our products throughout the smaller partners of the power industry.

We focused on static power analysis to detect events that can trigger cascading, and thus a catastrophic blackout. Our first model leads to a mixed integer nonlinear programming (MINLP) problem, where nonlinearity comes from the power flow equations and the blackout severity metric that we have developed, while the integer variables correspond to identifying broken power lines. This leads to an inherently difficult problem to solve. We have used a continuous relaxation technique to solve small to medium sized problems, and we are currently working on enhanced algorithms.

In one of our approaches, we restrict the power flow analysis to active power by fixing voltages at their nominal values. This reduced model still leads to a MINLP problem, but our analysis of the structure of an optimal solution identified a special combinatorial property, which we exploited to reduce the problem to a pure combinatorial problem, which is relatively easy to solve. We successfully applied our techniques to benchmark power systems with more than $13 \mathrm{~K}$ nodes and $16 \mathrm{~K}$ lines, where the solution times were only in the order of minutes.

In another approach, we proposed a method to measure the criticality of a line. The main advantage of this approach is that it can provide global information about the system, which can be used to identify multiple vulnerabilities, as opposed to the previous approach, where we identify only the most critical vulnerability.

We are also exploring other areas, where our results might be useful, including subsurface flows on fractured rock, biological networks, and vulnerability analysis of other critical infrastructure, such as the transportation network. 


\section{Statistical Feature Modeling for Scientific Data Via Basis Decomposition Principal Investigator: Raquel Romano}

\section{Project Description}

This project applies machine learning algorithms to problems in scientific data analysis. In contrast with many task-specific scientific data analysis methods, machine learning techniques are data-driven and easily adapted to changing data sets and experimental conditions. Statistical models are built directly from observed and simulated data and then queried in order to answer questions in the domains of radiation biology, astrophysics and climate modeling. Supervised learning techniques are used to detect and quantify phenomena of interest from microscopy and astronomy imagery, e.g., the detection of DNA-repair proteins in high-throughput biology and the recognition of supernovae and other celestial objects in large-scale digital sky surveys. Unsupervised learning algorithms extract structural (e.g., spatial, temporal, spectral) patterns and trends from observed and simulated data, and are useful when the behavior of the signal is due to both deterministic processes and stochastic processes that introduce uncertainty into measured values. Linear and nonlinear decomposition methods are applied to synthetic and observed Type Ia supernovae spectra in order to characterize the spectral features that describe a spectrum's variability due to physical parameters such as age or luminosity. Statistical models of climate simulation data at multiple scales are investigated in order to characterize the spatiotemporal patterns in simulations run under different external forcing scenarios.

\section{Accomplishments}

The first year focused on supervised and unsupervised methods for analyzing protein signals in microscopy imagery. In the second year, we have collaborated with the Nearby Supernova Factory at LBNL to deploy a fully integrated, state-of-the-art classifier, the Support Vector Machine (SVM), into the pipeline that searches $80 \mathrm{MB}$ of imagery per night for early Type Ia supernovae. Performance rates of $92 \%$ signal detection at a corresponding $99.1 \%$ rejection rate of false candidates (e.g., noise and image artifacts) are achieved, where an error rate of less than $1 \%$ is essential for reducing the burden of manual scanning of potential candidates. By rank-ordering all incoming candidates per night by classifier confidence, the human workload has been reduced by a factor of 8 . Linear and nonlinear decompositions of time- and luminosity-varying supernovae spectra are being investigated in order to find a data-driven, low-dimensional representation of observed spectra that corresponds to physically meaningful parameters, identifies which spectral features are associated with these parameters, and may be used for matching observed and synthetic spectra. In the climate domain, high-resolution ensemble runs of the NCAR CCSM 3.0 Community Atmosphere Model (CAM) have been analyzed to compare the quantity and intensity of tropical cyclones under past and future emission scenarios. Statistical models are being developed to understand the short-term and longterm relationships between intense tropical storms and other atmospheric variables. 
Extended First Order System Least Squares Finite Elements Principal Investigator(s): Jon Wilkening

\section{Project Description}

The purpose of this project is to develop numerical methods in the least squares finite element setting for adjoining special basis functions to the finite element space in order to capture features of the solution which are not accurately represented by the usual basis functions. We intend to apply these methods to problems in solid mechanics involving stress singularities (e.g. crack propagation and grain boundary evolution), and to problems in fluid mechanics involving coupling with elastic or inertial bodies.

This project may be divided roughly into three key components. First, the asymptotics of the solution near corners and interface junctions must be understood and a stable way of computing basis functions to represent the singular part of the solution must be devised. Second, these basis functions must be adjoined to the finite element space and an algorithm for computing the stiffness matrix must be developed to avoid the difficulties which are associated with the presence of the singularities. This should be done in the context of interesting physical problems so that the method is of practical use. And third, a strategy for performing an error analysis in the presence of the singular functions must be developed in order to identify the quantities that affect the error and show that the method can be trusted for other application problems which we have not yet explored empirically.

Accomplishments

We have succeeded in developing an algorithm for computing stable representations of singular functions describing the asymptotic behavior of an elliptic system near a corner or interface junction. Stability here relates to the presence of logarithm terms in the asymptotic expansion when a certain characteristic determinant has multiple roots. If the geometry or equations are perturbed slightly, the roots will typically split and the singular functions will change form. The goal is to find basis representations that smoothly recover the logarithm terms as the perturbation is reduced to zero so that the coefficients in the asymptotic expansion with respect to this basis don't blow up in the limit. Our method is based on orthogonalization with respect to a certain Riemannian metric to automatically choose the correct stable linear combinations of singular basis functions without needing to find or determine the structure of the singularities of any nearby critical geometries.

We have also succeeded in developing a streamlined way to adjoin these singular functions to the finite element space and compute the stiffness matrix without employing special purpose quadrature rules for overlapping singular functions in the corners. This is possible because the singular functions satisfy the PDE; hence, they are invisible to the FOSLS functional except in the fringe region where they transition to zero. But in this region, they are not singular and can be integrated using the same Gaussian quadrature rules used for the regular basis functions.

We are currently analyzing the error of the method to identify the situations in which it can be guaranteed to perform well. 


\section{Earth Sciences Division}

\section{LB06001 \\ Microarray technology for fungal identification: Its utility from the environment to homeland security, biomedicine, and beyond \\ Principal Investigator: Gary Andersen}

\section{Project Description}

This project will yield biomarker gene sequences from a unique collection of fungi collected around Chernobyl, Ukraine over 16 years following the nuclear power plant accident in 1986, and also will provide the nucleus for a web-based database of fungal biomarkers available to other researchers. Fulfillment of these two objectives will result in the development of a prototype DNA microarray specific for fungal detection and identification. Such a microarray has many uses, from environmental to medical applications and many advantages over current technologies. The database constructed through this work will be used to select DNA probe sets that will allow the detection and identification, to the level of genus or species, of fungi from any source material. This microarray could greatly advance our knowledge of the role fungi play in important matters such as bioremediation, carbon sequestration, biomass degradation, and human diseases in addition to providing a monitoring tool for protection against bioterrorism.

The project foresees the sequencing of variable regions of the large subunit nuclear ribosomal RNA gene and the preceding two internal transcribed spacer (ITS) regions of up to 2000 fungi collected from the Chernobyl Exclusion Zone. The sequences obtained will be used to develop a database similar to our previously developed bacterial biomarker sequence database (http://greengenes.lbl.gov/cgi-bin/nphindex.cgi), which will be available to other researchers online. The compiled sequences will then be used to select DNA sequences unique to fungal families, genera and species. These DNA sequences will provide the data necessary to construct a prototype microarray for detecting and identifying fungi from collected samples.

\section{Accomplishments}

During the current reporting period, we have preferably sequenced the variable region, D1/D2, of the 28S large subunit RNA-coding gene of filamentous fungi from the Chernobyl. The focus this time was on organisms that were either collected directly from the failed unit-4 of the nuclear power plant or within the $10-\mathrm{km}$ exclusion zone. High quality sequencing requires high quality genomic DNA. Therefore, we experimented with several commercially available genomic DNA extraction kits and sample treatment methods. Good results were obtained using ballistic cell disruption (FastPrep) and PureGene treatment. Additionally, in a new collaboration, we have sequenced the D1/D2 domains of the 26S large subunit ribosomal RNA genes of yeasts that were isolated earlier by the late Dr. Herman Phaff. These cultures are currently maintained in the culture collection at UC Davis, and represent so far unpublished, new yeast species. Raw sequences are edited, blasted against available public databases, and aligned for taxonomy and phylogenetic comparison.

In addition to documenting newly discovered fungal genomic diversity from a recently disturbed environment, we have organized gene sequences for ribosomal genes from the public database. Our largest collection is composed of over $21,00018 \mathrm{~S}$ rRNA full-length gene sequences, $90 \%$ of which had high similarity to type strains and were aligned into a global MSA. The remaining $10 \%$ contained only short regions for alignment. Greater scrutiny was required compiling the MSA for comparing the D1/D2 region of the $28 \mathrm{~S}$ rRNA gene. Of the 62,000 records analyzed, we have selected only 11,000 that contained sufficient nucleotide information for reliable taxonomic placement. The database represents 1267 and 1761 fungal taxa documented by the $18 \mathrm{~S}$ and 28S genes, respectively. For 906 of these taxa, data from both genes are linked. These accomplishments have brought about the largest fungal DNA biomarker database, to our knowledge. The alignments can be viewed online with restricted access in our pre-release database.

We are in the process of modifying our custom microarray design software to select sets of $25 \mathrm{mer}$ probes for each taxa for sequence-specific detection and identification. The resulting probes will be synthesized upon the prototype microarray. 


\section{Scientific Basis for Advanced Geologic Storage Technologies Principal Investigator: Sally M. Benson}

\section{Project Description}

The purpose of this project is to develop the scientific basis for the next generation of technology for geologic storage of carbon dioxide in deep underground formations. Today, geologic storage technology relies on a combination of physical and chemical processes to keep $\mathrm{CO}_{2}$ trapped securely underground. Initially, physical trapping beneath low permeability barriers is extremely important because the low density and viscosity of $\mathrm{CO}_{2}$ tend to drive $\mathrm{CO}_{2}$ upwards towards the land surface. Over longer timescales, on the order of hundreds to thousands of years, the $\mathrm{CO}_{2}$ dissolves into the formation fluids, becomes trapped by capillary forces within the storage formation, and eventually can form carbonate minerals. Once dissolved, immobilized by capillary forces or mineralized, $\mathrm{CO}_{2}$ is in essence, stored permanently. The purpose of this project is to identify physical, chemical and biogeochemical approaches that can be used to accelerate the formation of permanently stored $\mathrm{CO}_{2}$.

The approach taken is to use numerical simulations of multi-component, multi-phase, reactive chemical transport of $\mathrm{CO}_{2}$ to test various approaches for accelerating dissolution, capillary trapping, and mineralization. TOUGH2, TOUGH-Fx,TOUGHREACT and developmental versions of these codes will be used to carry out these simulations. In addition, laboratory experiments will be carried out to quantify the extent of capillary trapping of $\mathrm{CO}_{2}$ in sedimentary rocks. Possible methods to accelerate dissolution, capillary trapping and mineralization include manipulation of the injection geometry and flow field to increase contact area between the $\mathrm{CO}_{2}$ and formation and the addition of cocombustion products such as $\mathrm{H}_{2} \mathrm{~S}, \mathrm{SO}_{2}$ and $\mathrm{NO}_{2}$. Injection of brine may also accelerate capillary trapping. These and other ideas will be tested.

Accomplishments

The major accomplishments of this research include demonstration of the following:

- Capillary trapping effectively immobilized $\mathrm{CO}_{2}$ saturations up to $22 \%$ of the pore volume of the rock during post-injection imbibition of brine;

- Trapping by all mechanisms is most effective when the $\mathrm{CO}_{2}$ is injected into the deepest parts of the geological storage reservoir;

- Trapping by dissolution and capillary trapping is most effective in lower permeability storage reservoirs;

- Cyclical injection of $\mathrm{CO}_{2}$ can accelerate capillary trapping;

- Use of horizontal injection wells and multiple injection wells accelerates trapping by all mechanisms; and

- Thirty years after injection stops, more than $90 \%$ of the injected $\mathrm{CO}_{2}$ can be trapped by a combination of capillary trapping and dissolution under the conditions investigated in these studies.

The overall conclusion based on this work is that with careful design of the injection system, the combination of capillary trapping and dissolution act to quickly immobilize $\mathrm{CO}_{2}$ - thereby reducing the risk of long term leakage. 


\section{Autonomous Sensors for Ocean Dissolved Organic Matter \\ Principal Investigators: Jim Bishop, Paul Luke}

\section{Project Description}

Dissolved organic carbon (DOC) in seawater constitutes a large, dynamic, yet poorly quantified reservoir of carbon in the oceans. Changes in the concentration of DOC reflect transformation of carbon between inorganic and organic, and between dissolved and particulate species. DOC changes may thus be a critical yet independent measure of changes in the biological pump in the ocean or of changing carbon sequestration in the ocean. DOC presently must be measured in shore based laboratories on ship collected water samples.

Here, we propose development of a novel autonomous ocean-profiling sensor for real time detection of dissolved organic carbon in seawater. Such a sensor when combined with other LBNL pioneered sensors will allow -- for the first time -- fully autonomous quantification of all products of marine photosynthesis on the very fast time scales of marine photosynthesis. Our effort supports current national initiatives for carbon prediction, management, and assessment.

Marine biofouling begins with adsorption of an organic film on a fresh surface. The process initiates on timescales of seconds. Our sensor concept is to present to the environment a pre-cleaned organic-free surface purposefully engineered for non-specific adsorption of dissolved organic matter from seawater. The developing organic film will be monitored for seconds, the surface will be cleaned and the measurement process repeated.

\section{Accomplishments}

In FY05 we constructed a new apparatus to measure changes of surface (double-layer) capacitance of an electrode array due to organic adsorption reactions. The new apparatus allowed improved investigation of electrochemical cleaning. Our batch addition experiments using artificial seawater at pH 13 (adjusted using $\mathrm{NaOH}$ ) demonstrated detection limits of 6 and 0.5 $\mu \mathrm{M} C$ for sucrose and natural DOC (supplied by Dr. D Repetta, at Woods Hole Oceanographic Institution), respectively.

This year (FY06) we achieved a major project goal: DOC detection at neutral $p H$ in artificial seawater. This means that the sensor can be operated in the environment without chemicals. The detection limit we achieved $(<6 \mu \mathrm{M})$ can be lowered further.

The apparatus displayed a slow drift (over hours) in its baseline which presently makes it difficult to determine the absolute DOC concentration without a suitable reference or ability to add or remove DOC. For this reason, most of our effort was spent on understanding and addressing long term stability issues; investigation of electrode cleaning strategies was part of this work. Thermal effects were briefly investigated and shown to be manageable. Sensitivity to variations of dissolved $\mathrm{O}_{2}$ in solution was documented and instrumental measurement settings can be chosen to avoid this interference. Long term drift has been reduced but not eliminated. The instrument we used has excessive noise from AC line voltage interference, which prevented us from observing small capacitance signals. Experimentation with more sophisticated instrumentation for capacitance measurement is under way. We believe that greater sensitivity and stability can be realized using this instrument.

We remain confident that the capacitance measurement approach can achieve adequate sensitivity $(\sim 1 \mu \mathrm{M})$ for detection of oceanic DOC at natural concentration levels. 


\section{An investigation of the microbial processes involved in a biological fuel cell PI - John D. Coates}

\section{Project Description:}

In a microbial fuel cell (MFC) bacteria transfer electrons derived from the catabolism of various alternative compounds onto an electrode (anode). The electrons are subsequently conducted through an electrical circuit towards a cathode in a separate chamber thus generating energy to do work. Chemotrophic biofuel cells are based on the ability of chemotrophic microorganisms to generate reducing equivalents through the oxidation of organic or inorganic chemicals and pass electrons onto an electrode either directly or indirectly through an electron shuttling compound. Many organic and inorganic substrates can be converted into electrical energy in biofuel cells, and the utilization of a given compound is dependent on the metabolic capability of the particular organism on which the fuel cell is based. The goals of this project are to investigate the microbial processes in energy generation in both the anodic and cathodic chambers of an MFC.

\section{Accomplishments:}

A. Research on the anodic chamber. Our recent studies on MFCs focused on investigating the operation and microbiology associated with thermophilic MFCs operating at $55^{\circ} \mathrm{C}$. Over 100-day operation, these MFCs were highly stable and achieved a maximum power density of 38 $\mathrm{mW} . \mathrm{m}^{-2}$ and a columbic efficiency of $83 \%$ with acetate as the sole electron donor. As part of our studies we enriched and isolated a novel organism, strain S2E, from samples collected from an operating MFC. Phenotypic characterization revealed that strain S2E was capable of thermophilic dissimilatory reduction of insoluble electron acceptors such as amorphous Fe(III). Strain S2E was also capable of reducing various soluble forms of Fe(III) including Fe(III)citrate, $\mathrm{Fe}(\mathrm{III})$-pyrophosphate, and $\mathrm{Fe}(\mathrm{III})$ chelated with nitrilotriacetic acid. In addition to $\mathrm{Fe}(\mathrm{III})$, strain S2E also grew by aerobic respiration on LB broth, and anaerobically with nitrate or 2,6-anthraquinone disulfonate, an analog for the quinone moieties of humic substances. As with most other described Fe(III)-reducing organisms, no growth or reduction was observed with sulfate as the sole electron acceptor. Analysis of the complete $16 \mathrm{~S}$ rDNA sequence revealed that strain S2E was a member of the Geobacillus genus in the gram-positive phylum of the Bacteria. To our knowledge, this represents the first organism isolated from a thermophilic microbial fuel cell and also the first representative of this genus shown to be capable of dissimilatory iron reduction. In general the genus Geobacillus is described as a gram positive, spore forming, obligately thermophilic, facultative anaerobes. Whether or not this organism can grow through the dissimilatory reduction of the anode in the MFC remains to be determined.

B. Research on the cathodic chamber. Our lab have also focused on the cathodic chamber, its potential for biological activity, and the final oxidant utilized. To this end we focused our efforts on stimulating the biological reduction and treatment of perchlorate by perchlorate reducing bacteria (PRB) in the cathodic chamber. We developed a novel reactor based on electrical stimulation of PRB at the cathode which allows respiratory metabolism while limiting growth. Our fed-batch studies showed that native PRB could use electrons from a graphite cathode poised at $-500 \mathrm{mV}$ (vs. $\mathrm{Ag} / \mathrm{AgCl}$ ). As part of these studies we isolated a novel organism, Dechlorospirillum strain VDY. Strain VDY readily reduced $100 \mathrm{mg} . \mathrm{L}^{-1}$ perchlorate in a mediatorless batch bioelectrical reactor (BER) in 6 days. Up-flow BERs seeded with active cultures of strain VDY continuously treated waters containing $100 \mathrm{mg} . \mathrm{L}^{-1}$ perchlorate with almost $100 \%$ efficiency throughout their operation achieving an unoptimized volumetric loading of $60 \mathrm{mg} . \mathrm{L}^{-1}$ reactor volume.day ${ }^{-1}$. The mediatorless BERs also treated low level perchlorate (100 mg.L ${ }^{-1}$ ) influent as well as mixed-waste influents more typically found in the environment containing both nitrate and perchlorate. 
Micro-characterization and chemical micro-dynamics of atmospheric mineral dust

Principle Investigators: Donald J. DePaolo and John N. Christensen

\begin{abstract}
Project Description
The objective is develop isotopic analysis and modeling techniques to better characterize the sources and transport of atmospheric mineral dust and the chemical and physical transformations that affect its properties. Mineral dust is an important component of the atmospheric aerosol budget. It is now recognized that dust travels long distances in the atmosphere, even to the point that a substantial fraction of the dust present over the western U.S. comes from Asia. Dust therefore constitutes a means of intercontinental material exchange and has important health and security implications. Dust also affects the atmospheric radiation balance and therefore is a critical component of the global climate change. Our objective is to evaluate the extent to which isotopic techniques can be used to fingerprint dust sources and evaluate how these techniques can be best applied. A critical aspect of this is sampling techniques. Another aspect is microchemical analysis and imaging. A third component of the study is the development of Lattice-Boltzmann codes for modeling the micro- physics and chemistry of dust grain surfaces. This project combines lab capabilities in isotopic geochemistry, synchrotronbased analysis and imaging, atmospheric transport, and computational physics and chemistry.
\end{abstract}

\title{
Accomplishments
}

In the second year of the project we have, in collaboration with researchers at U.C. Davis and the California Air Resources Board, completed the first phase of tests of the characterization methods, which involve a study of filters from Heifei, China, taken in Spring 2002 during a dust storm that eventually carried dust to the western U.S. Strontium isotopic measurements, combined with chemical analysis, clearly show that the aerosol load has three sources - local eastern China lowlands, the Western MongolianNorthwest China deserts, and the marine atmosphere over the adjacent ocean. Tracer results can be compared with air mass trajectories and are in excellent agreement. Isotopic analyses of $\mathrm{Nd}, \mathrm{Pb}, \mathrm{U}, \mathrm{S}$, and $\mathrm{O}$ are still in development, as are sample analysis protocols, including serial leaching and dissolution approaches to removing dust from the collection devices.

As a first step toward simulating chemical reactions at dust particle surfaces we have adapted an existing 2D Lattice Boltzmann code for the simulation of microscale kinetics of crystal growth from a fluid medium. This code has been modified to handle multiple reacting species, to conserve mass, and to accommodate the boundary conditions that are necessary to appropriately simulate surface reactions. We have applied it to the problem of mass-dependent isotopic fractionation associated with water vapor deposition on ice crystals in clouds. We can now simulate both $18 \mathrm{O} / 16 \mathrm{O}$ and $\mathrm{D} / \mathrm{H}$ isotopic fractionation and we can scale the results using kinetic theory and measured diffusion coefficients. 


\section{The Behavior and Impact of Nanoparticles in the Environment}

Principal Investigator: Benjamin Gilbert

\section{Project Description}

Natural nanoparticles are virtually ubiquitous products of inorganic precipitation and weathering, as well as both sources and products of microbial metabolism. They participate in numerous biogeochemical processes but their impact depends factors such as redox behavior and transport properties that are generally not well characterized. Research in the following areas is particularly relevant for understanding the fate of nanoparticles in the environment, and for beneficial applications of nanoscience in the application and monitoring of remediation technologies: 1. transport behavior of natural and anthropogenic oxide nanoparticles; 2 . stimulation of bioremediation with ferric iron nanoparticle amendments; and 3. Investigation of size effects on the chemical properties of nanoparticles.

Accomplishments

Further research was performed on the synthesis and characterization of water-soluble ferric iron oxyhydroxide $(\mathrm{FeOOH})$ nanoparticles for stimulating the bioremediation of heavy metal and organic contaminants in groundwater. After demonstrating that the solubility of FeOOH nanoparticles could be improved in circumneutral water, we informed the LBNL Patent Dept of this novel idea. However, further research indicated that the nanoparticles coated in succinic acid did not stay suspended indefinitely. Thus we expanded our studies to investigate alternative solubilizing ligands.

This topic was the basis of an unsuccessful proposal application to the DOE ERSP. However, the reviews were overall good, and we are optimistic that with additional preliminary data this research may be funded. In particular, laboratory experiments are planned to test the growth of Geobacter using coated goethite nanoparticles as the sole energy source.

An important goal of the LDRD is to address the electronic and chemical properties of natural nanoparticles. We used soft-x-ray absorption and emission spectroscopy at the LBNL ALS to reveal exciting differences between the band edge states in a variety of ferric iron oxide and oxyhydroxide nanoparticles (hematite, maghemite and goethite). Our most important observation is that the absolute energy positions of the valence band (VB) and conduction band (CB) can be shifted in nanoparticles relative to the bulk material. Both hematite and maghemite nanoparticles exhibited band gap opening $(\sim 0.25 \mathrm{eV})$, while a closing of the gap was observed in goethite nanoparticles.

The origin(s) of these effects are presently unclear, but they are of direct relevant to our understanding of the reactivity of natural nanoparticles. Moreover, these observations indicate that very small ferric iron oxide nanoparticles may provide genuinely new materials from solar energy utilization. These observations strongly indicate that the electrochemical potential of photogenerated electrons and holes will be enhanced in these materials. 
Compositional and Functional Analysis of Cell-Wall during Metal-Bacterial Actions Principal Investigators: Hoi- Ying N. Holman, Ping Hu, Sharon Borglin

\section{Project Description}

The purpose of this project is to initiate the development of a dual imaging technology at the Advanced Light Source. It builds upon the novel tunable vacuum ultraviolet (VUV) nanoimaging of biomolecules (developed by the Ahmed Group) at the Chemical Dynamics Beamline and our Fourier transform infrared spectromicroscopy of live cells at the Infrared Beamline. These results will enable us to further the development of existing molecular microprobe tools for characterizing bioremediation of heavy metals. Data generated also would allow us to help creating computational tools to infer both immediate and long-term stability of these biologically transformed metal ions in subsurface environments, which will enable a more realistic assessment of sustainable bioremediation technologies.

We will develop a cell-wall-like system in which its physical and chemical properties can be rigidly controlled for investigating biomolecule-metal interactions. After establishing these model systems, we will use them as test beds for the development of the VUV nano-imaging, and for the extension of the existing infrared micro-imaging technology. We will test the existing instrumentation components and operation parameters, and will modify them if necessary. These data will also be used to extend existing molecular dynamic algorithms, which will enable a more in-depth analysis of the dynamics of an interacting system.

Accomplishments

Dr. Kristina N. Woods (Ph.D. Biophysics, Stanford University) joined us as a full-time postdoc to work with the PIs on the project.

Our most significant accomplishment has been to develop a reproducible protocol for preparing a liposome-metal interacting system that can model after the interactions between chromium(VI) and the outer membrane of a Gram-negative bacterium. This protocol has been successfully used to prepare three different types of membrane-like liposomes. We are in the process of extend ing this protocol to the preparation of a cell-wall-like model system, which will greatly aid our meeting of the project goal.

Another significant accomplishment has been to develop a method that can deliver membrane-like liposome particles in suspension reproducibly into the VUV Aerosol Endstation. We have analyzed the physical properties and chemical composition of the liposome particles. As we had hoped, the method does not alter the physical properties of the membrane-like liposome particles. We are now varying the experimental parameters, and are hopeful that we can establish a sample delivery method that would not alter the chemical properties of the membrane-like liposome particles. We are hopeful that this will be applicable to the cell-walllike system.

We also have successfully adapted our existing experimental protocol at the Infrared Beamline Endstation for characterizing liposome-metal interacting systems.

Finally, we are in the process of evaluating molecular dynamic algorithms for performing ab-initio calculations of the system. 


\title{
The California Water and Energy System: An Approach for Addressing Future Crises
}

\author{
Principal Investigators: Norman L. Miller and Larry L. Dale \\ Collaborators: California Department of Water Resources, California Energy Commission
}

\section{Project Description:}

The purpose of this LDRD project is to understand how natural processes and human intervention interact to influence water supply and water quality, and the sensitivity of the California water supply system to potential disruptions. We have developed advanced tools that provide reliable quantitative evaluation of risks under different future climate scenarios, and have integrated physical, water quality, and allocation modeling to quantify water supply reliability under changing conditions.

Our specific goals are: (1) Quantitatively understand the climate drivers impacting mountain front recharge, snowmelt runoff, and net infiltration in the Sierra Nevada and Central Valley. (2) Investigate the sensitivity of the water table to these inputs and to pumping withdrawals. (3) Adapt existing geophysical monitoring techniques for characterizing the depth distribution of groundwater contaminants. (4) Develop a regional resource management model to demonstrate economic tradeoffs between agricultural and environmental groundwater pumping, incorporating long-term aquifer degradation. (5) Develop a crop production model to better-forecast agricultural electricity and groundwater use. (6) Determine the extent to which existing planning models can be used to investigate system response under different hydrological conditions.

Two spatial scales are considered to understand detailed physical processes and policy implications. These scales are the State level (California and the California Central Valley), and the Local level (Merced River Basin west to the adjacent Grasslands Ecological Area). At present, there is no other research group that brings together these core components. Work on both these scales has resulted in a much better understanding of potential uses of groundwater and surface water, and tradeoffs between electricity and water supply. A series of papers and research projects resulting from this work are being conducted in collaboration with the CDWR and CEC, described below.

\section{Accomplishments:}

Our accomplishments include generation of a 100-year baseline climate and a perturbed climate with an imposed 25-year drought for testing the groundwater recovery rates at the Merced BasinGrasslands Ecological Area. We also advanced the LBNL Regional Climate System Model to include a state-of-art land-surface/shallow subsurface model (CLM3) with advanced snow and hydrologic processes, and developed a LBNL/TOUGH2 groundwater module with confined/unconfined aquifer and saturated/unsaturated conditions with initial CLM3 coupling. We also performed modification of the Integrated Groundwater Surface water Model V.2 for the Merced River Basin and a series of imposed drought simulations for the entire Sacramento-San Joaquin Valley.

Additionally, we have collected baseline water supply and use data for agricultural and urban districts in the Central Valley, formulated and calibrated an electricity groundwater-pumping logit model and obtained estimates of historical groundwater pumping regions in the Central Valley. These results have been implemented into the DWR California Central Valley Simulation (C2VSIM) model. A series of papers and research projects resulting from this work are being conducted in collaboration with the CDWR and the CEC. The follow up projects include funded research to help the CEC prioritize future water energy research projects and better estimate impacts of change in water supply on groundwater, cropping patterns and electricity use in the Central Valley. 


\section{Interrelation of Global Warming and Hydrate Dissociation in Oceanic Accumulations George Moridis and Matthew Reagan}

\section{Project Description}

Extensive deposits of methane hydrate occur in deep ocean sediments and in the permafrost, where low temperatures and high pressures exist to promote formation and stability. Current estimates report total hydrate deposits of $10^{15}$ and $10^{19} \mathrm{~m}^{3}$ worldwide. An increase in the temperature of the ocean water in contact with the seafloor could induce hydrate dissociation, leading to large-scale methane release into the oceans and atmosphere. Methane is a powerful greenhouse gas, and there is considerable concern that the rapid injection of large quantities of methane into the atmosphere could have dramatic climatic consequences. Such hydrate dissociation has been proposed as a mechanism for rapid and significant climate change (the "Clathrate Gun" hypothesis).

The purpose of this study is 1) to investigate the effect of rising water temperatures on oceanic hydrate accumulations and to determine under which conditions carbon release is likely to occur, 2) to estimate the quantity of carbon that could escape into the atmosphere, accounting for hydrate thermodynamics, fluid transport, and benthic chemistry, 3) to couple hydrate dissociation to global climate models to determine the overall effect on global climate, and 4) to test the hypothesis that oceanic hydrates may contribute to rapid climate change.

Accomplishments

We have investigated, through numerical simulation, the effects of ocean warming on the stability of oceanic hydrate deposits using the TOUGH-Fx/HYDRATE code and the CCANDI diagenetic model. We have identified critical chemical and biochemical reactions within the benthic environment, including anaerobic oxidation of methane to carbon dioxide and the formation of solid carbonates, that regulate the proportion of methane and carbon dioxide released into the environment.

For deep, cold, and sparse hydrate systems, we find that such deposits are relatively insensitive to moderate temperature changes. Methane release increases moderately, staying below limits of anaerobic methane consumption with fluid flow velocities that would not hinder formation of immobile carbonate deposits.

For deposits at moderate depths (known Gulf of Mexico deposits) we find significant response to temperature changes. Warming of $3-5^{\circ} \mathrm{C}$ over a century produces an initial surge of fluid and gas production, followed by gradual but continuing release of methane into the upper sediment layers until the deposit is depleted. The ability of seafloor chemistry to accommodate this methane is dependent on the local rate of methane transport and the velocity of fluid flow. Scenarios with the greatest amount of methane release produce more methane than anaerobic oxidation can consume, and high fluid velocities inhibit carbonate formation and push gaseous methane through the thin reactive zone into ocean waters.

Finally, for cold, shallow arctic hydrates, temperature increases can produce rapid hydrate dissociation with large, continuous production of gas and high fluid flow rates, suggesting rapid, "explosive" release of methane gas bubbles and hydrate-derived fluids.

We are in the process of testing an integrated thermodynamic, transport, and biogeochemical model that can be coupled to advanced ocean circulation and global climate models. 


\section{A New Technology for Permeability Enhancement for Natural Gas Extraction in Tight Reservoirs \\ Principal Investigator(s): Seiji Nakagawa and Andrea Cortis}

\section{Project Description}

With the dwindling supply of foreign and domestic oil and the push to reduce $\mathrm{CO} 2$ emissions, natural gas is destined to be one of the premier energy sources by the next decade. A significant part of technically recoverable gas is believed to be trapped within very lowpermeability, "tight" gas reservoirs. The primary objective of this project is to develop an entirely new approach for enhancing the production of natural gas in tight reservoirs. The approach employs a self-growing, high-permeability "slot" that is driven from a borehole. The slot is advanced through a coupled hydro-mechanical process in which large stress concentrations around the borehole (or the advanced slot) in concert with high velocity fluid circulation combine to advance a permeable plane through a repeated process of stress concentration $\rightarrow$ rock failure $\rightarrow$ hydraulic debris removal. This method is potentially superior to the conventional hydraulic fracturing method to enhance formation permeability, because the orientation of the slot growth is optimal to encounter preexisting fractures.

Recent laboratory experiments have confirmed the formation of slot-like high permeability planes in high porosity rocks. The same process may be possible in low porosity (tight) rocks, if right physical conditions are realized. This project will employ an experimental approach aimed at quantifying the conditions (stresses and fluid velocities) required to nucleate and propagate slot-like failure planes in tight, low-permeability reservoir rocks. Based on the experimental results, critical parameters for controlling the growth of the slot failure will be identified and their quantitative relationships will be examined using available rock failure models in combination with hydrodynamic disturbances on the failing rock. Concurrently, numerical simulations will be conducted to examine the process of rock failure and debris transport at the grain scale and to study quantitatively the evolution of the slot geometry and flow pattern.

\section{Accomplishments}

We developed new laboratory techniques to study the growth of a "slot-shaped" borehole breakout in tight sandstones. These are 1) a fabrication technique for synthetic low-permeability sandstone samples (porosity down to about 7\%) to conduct controlled experiments and 2) a simple experimental technique to induce borehole breakout in a small, high-strength core samples without large confining stresses. Initial tests on very low porosity, natural rock cores (limestones) indicate that, compared to high-porosity rocks, much stronger hydrodynamic force may be required to propagate a breakout in tight rocks. Currently, we are building an experimental setup to apply a low to medium-pressure water jet to the sample to examine its effect on breakout growth.

On the modeling side of the effort, we conducted 2D Finite Element (FE) simulations to evaluate the force and the torque generated by a water jet stream on a simulated sandstone wall consisting of spherical (cylindrical) grains. This numerical model solves Navier-Stokes flow problems at different Reynolds numbers to study the effects of the turbulence at the wall boundary. The resulting tangential and normal stress at the grain boundaries are used to obtain the total force and torque acting on individual grains, which will be used as an input to a Discrete Element (Particle) Model to compute stress and evaluate failure within the solid phase. 
Coupled Modeling of Hydrology, Nutrient Cycling, and Vegetation: Applications to Water Quality and Water Balance

Principal Investigators: Curt Oldenburg, Bill Riley, Norm Miller, Carl Steefel

\section{Project Description}

This joint Earth Sciences Division (ESD) and Berkeley Water Center (BWC) LDRD project is focused on enhancing the ability of researchers to analyze local- to regional-scale water, nutrient, and elemental fluxes and cycling using the TOUGH2 family of codes as interpretive and predictive tools. The motivation for the effort is the need to predict and interpret the effects of climate and land-use change in California's Central Valley (CV). The technical objective is the further development of a coupled TOUGH2-CLM3 code to include reactive biogeochemical transport capabilities. This new capability will then be applied to address the question: What are the effects of regional climate change in California on the dynamics of the nitrogen cycle in the Central Valley (CV)?

The approach to addressing the question posed is to develop a new simulation capability starting from existing established codes. The existing TOUGHREACT code will be modified to treat nitrogen biogeochemical networks and cycling by including time-dependent biomass, parallel reaction networks, and explicit accounting of energy used in biomass growth versus metabolism. The existing CLM.T2 code will be modified and further evaluated to include the components needed by TOUGHREACT. These two bottom-up and top-down modeling capabilities will be coupled to produce a new modeling capability for nitrogen cycling that includes hydrobiogeochemical controls as driven by climate, land-use, and hydrologic change.

\section{Accomplishments}

During our first year (FY06), LBNL researchers initiated technical work in the project by adding nitrogen species to TOUGHREACT $(\mathrm{Xu}, 2006)$ and verifying the TOUGH2-CLM3 code (Pan et al., 2006). Specifically, new TOUGHREACT capabilities for intra-aqueous kinetic reactions and biodegradation were verified by comparison to published results of denitrification and sulfate reduction. A general multi-region model for subsurface hydro-, bio-, geologic-structures was used. The TOUGH2-CLM3 code was tested and verified against 18 years of observations in the Valdai watershed, Russia. It was found that the coupled model greatly improves the predictions of the water table, evapotranspiration, surface temperature, and moisture in the top $20 \mathrm{~cm}$ of soil. These two codes will form the foundation of our efforts in developing the coupled nutrient cycling modeling capability.

In addition, LBNL worked closely with the BWC to advertise and interview candidates for two post-doctoral positions that will be shared between LBNL and BWC. We hired two outstanding postdocs selected from a pool of over 30 qualified applicants. Prior to the arrival of the postdocs, we held a series of regular joint LBNL-BWC project meetings to coordinate the research, wrote a work plan, and developed a Twiki web collaboration site for the project. 


\section{Biogeochemical Reaction Rates and Pathways in Porous Media Principal Investigator: Carl Steefel}

\section{Project Description}

The purpose of this project is to dramatically increase our understanding of the scale dependence of biogeochemical and geochemical reaction kinetics in natural porous media. A second goal is to quantify the rates of precipitation and nucleation of important secondary mineral phases in soils and aquifers, a critical deficiency in current hydrogeochemical models. The present lack of understanding limits our ability to develop effective bioremediation schemes for contamination cleanup, to develop predictive models for $\mathrm{CO}_{2}$ sequestration in deep aquifers, and even to determine the fundamental controls on the rates of chemical weathering, an important long-term regulator of atmospheric $\mathrm{CO}_{2}$ levels.

The research approach is to collect baseline reaction rate data using conventional well-mixed flowthrough reactors and less conventional microfluidic reactive flow devices and then use these rates to interpret averaged or effective rates in porous media. As model systems, we are considering (1) the dissolution of calcite (a ubiquitous subsurface phase), (2) the abiotic and microbially-mediated reductive dissolution of Fe-hydroxides (important phases in bioremediation and natural attenuation of contaminants), (3) the dissolution of olivine (a model system with relevance to the problem of $\mathrm{CO}_{2}$ sequestration), and (4) clay formation at circumneutral $\mathrm{pH}$.

\section{Accomplishments}

We have significant results in three main areas: microfluidic reactive flow experiments, kaolinite precipitation and dissolution, and $\mu$ X-ray Tomography of Rock Weathering.

Microfluidic reactive flow experiments with calcite are being used to establish the comparison between rates determined in conventional laboratory flow-through reactors and rates determined in single pores. The experimental setup consists of a 500 micron thick wafer of pure calcite (Iceland spar) sandwiched between two ceramic supporting frames. A single cylindrical hole measuring 150 microns in diameter is drilled through the calcite and the supporting ceramic wafers. Inlet and outlet tubing is then connected to a low flow velocity syringe pump. Effluent total calcium concentrations, a measure of the extent of dissolution of the calcite in the pore, are compared with results from a $2 \mathrm{D}$ axi-symmetric cylindrical reactive transport model in which the reactive calcite is present only at the cylinder wall. The detailed flow field used as input to the modeling is taken from the analytical solution for flow in a cylinder.

In preparation for pore-scale kaolinite precipitation and dissolution experiments, we have conducted stirred flowthrough reactor experiments at $\mathrm{pH} 4$ and $25^{\circ} \mathrm{C}$ on Georgia kaolinite. The results to date demonstrate that, at least at this $\mathrm{pH}$ value, kaolinite precipitation and dissolution are fully reversible.

As part of our effort to understand the controls on the rates of reaction in natural porous media, we have undertaken $\mu X$-ray tomographic imaging of the reaction interface separating weathered from fresh rock to elucidate the mechanisms by which the weathering occurs. The tomography carried out on ALS Beamline 8.3.2 indicates that weathering results in the formation of sinuous dissolution channels that enhance both the porosity and its connectivity. This has been confirmed by analyzing the data with the code Percolate from the National Institute of Standards and Technology 


\section{Engineering Division}

LB05010

Novel Imaging Detectors for Materials and Biology Research

Principal Investigator(s): P. Denes and H. A. Padmore

\section{Project Description}

The purpose of this project is lay the groundwork for a new program in imaging detectors. LBNL's long experience in specialized detectors for particle physics and astronomy provide the technical foundation, and this project includes specific R\&D to realize high-impact, new types of detectors. We are focusing on improving the ubiquitous detector in synchrotron radiation research and electron microscopy, based on a scintillating phosphor fiber-coupled to a CCD. In order to improve sensitivity and live time, as well as enabling new kinds of dynamic experiments, we are developing a highspeed CCD which can be read out at the rate of $5 \mathrm{~ms}$ per megapixel while maintaining a dynamic range in excess of 14 bits. This combination of speed, dynamic range and number of imaging pixels is unprecedented. In addition, we are developing an innovative electron microscopy detector, which will not only be fast and sensitive, but provide excellent spatial resolution - particularly at the higher energies which are of interest for thick biological specimens.

Accomplishments

During FY06, we have demonstrated the principle of direct electron detection at energies typically used in Transmission Electron Microscopy. In particular, we have obtained spatial resolution at $200 \mathrm{keV}$ of $15 \mu \mathrm{m}$, with a signal-to-noise ratio of better than 8 without any cooling. Spatial resolution improves at higher energy, and with certain technology choices. We plan to demonstrate spatial resolution below $10 \mu \mathrm{m}$ in the coming year.

For the high-speed CCD, our FY06 efforts focused on the development of the very ambitious custom readout integrated circuit. Based on the concept developed for the SNAP experiment, the fast CCD IC runs ten times as fast, and packs four times the number of channels into the same space as the SNAP chip. Fabrication of the circuit started in July 2006, with testing to start in November 2006. Fabrication of the CCDs themselves also took place during FY06, with testing to take place in FY07. Finally, for the CCDs, we have developed an innovative all-silicon mechanical support, which allows a compact design that operates over a wide temperature range. When assembled, the camera will be capable of 400 frames per second with a 15 bit dynamic range at the input. 


\title{
Environmental Energy Technologies Division
}

LB05012

\author{
Constraining Ammonia Emission Inventories for Control of Air Quality \\ Principal Investigator: Marc L. Fischer
}

Project Description

Quantifying atmospheric ammonia $\left(\mathrm{NH}_{3}\right)$ concentrations and the $\mathrm{NH}_{3}$ fluxes between the land surface and atmosphere is required for effective control of aerosol concentrations affecting human health, climate, and visibility. In areas where $\mathrm{NH}_{3}$ is limiting aerosol concentrations, controls being contemplated on combustion related emissions of $\mathrm{NO}_{\mathrm{x}}$ and perhaps $\mathrm{SO}_{\mathrm{x}}$ will not be effective in controlling aerosol. Hence, measurement of $\mathrm{NH}_{3}$ concentration and flux is essential for development of scientifically sound aerosol control strategies.

Approach/Methods: Both improving $\mathrm{NH}_{3}$ emission inventories and understanding aerosol concentrations both require sensitive concentration and flux measurements over a wide range of meteorological conditions. To address these measurement needs, we developed an instrument that combines a fast response $\mathrm{NH}_{3}$ sampling system with a compact quantum cascade laser (QCL) spectrometer and supporting micrometeorological instruments.

Accomplishments: In FY 2006, we conducted two field campaigns encompassing a wide range of $\mathrm{NH}_{3}$ concentrations.

In March, 2006, we participated in the Milagro 2006 campaign, a joint Mexico-US (DOE, NASA, NSF) air quality intensive in and around Mexico City (http://www.eol.ucar.edu/projects/milagro/). In collaboration with scientists from the National Center for Atmospheric Research, we deployed the LBNL $\mathrm{NH}_{3}$ instrument at the T1 site $(10 \mathrm{~km}$ north of Mexico City). Surface level $\mathrm{NH}_{3}$ concentrations were characterized by a 10-20 ppb baseline with intermittent strong (50-250 ppb) peaks, associated with local sources.

Complementary column integrated $\mathrm{NH}_{3}$ measurements made by the NCAR team were linearly related to our ground-based measurements, with an offset indicating significant $\mathrm{NH}_{3}$ in upper level air. Combining measured $\mathrm{NH}_{3}$ and site meteorology, we show that the high $\mathrm{NH}_{3}$ concentrations are sufficient to allow production ammonium nitrate aerosol even at very low nitric acid concentrations $(<0.2 \mathrm{ppb})$.

In June, 2006, we measured $\mathrm{NH}_{3}$ at the UC Blodgett Forest in the Sierra Nevada. The measured $\mathrm{NH}_{3}$ was consistently low $(<2 \mathrm{ppb})$. This result was confirmed during a two day comparison against four 12-hour integrated filter samples analyzed by the Desert Research Institute (DRI). The low $\mathrm{NH}_{3}$ measured at Blodgett is likely due to the absence of any strong local sources and $\mathrm{NH}_{3}$ from the Central Valley being gradually deposited to vegetation in the area during up-slope transport. The average diurnal cycle of $\mathrm{NH}_{3}$ concentration is consistent with this hypothesis in that the maximum $\mathrm{NH}_{3}$ concentrations occur during the afternoon when flows of air arrive from the central valley. This suggests that $\mathrm{NH}_{3}$ emitted in the Central Valley may not reach far into the Sierra foothills during typical summer conditions. 
Exploring Electrochemical Arsenic Remediation of Drinking Water for Bangladesh

Principle Investigator: Ashok Gadgil, Co-PIs: Robert Kostecki, Venkat Srinivasan

\section{Project Description}

This project aims to investigate accelerated formation of $\mathrm{Fe}(\mathrm{OH})_{3}$ and $\mathrm{FeOOH}$ for affordably removing arsenic from drinking water in Bangladesh, where 60 million people drink arsenic-laden water from shallow wells. Approaches currently being tried or considered suitable for Bangladesh use rusted metallic iron (usually rusty nails) as a source of ferric hydroxide $\left(\mathrm{Fe}(\mathrm{OH})_{3}\right)$ or ferric oxyhydride $(\mathrm{FeOOH})$ which bind the arsenic into insoluble ferric arsenate. This approach is limited by the amount of $\mathrm{FeOOH}$ that can be produced in an affordable supply of nails. We intend to increase $\mathrm{Fe}(\mathrm{OH})_{3}$ or $\mathrm{FeOOH}$ production from metallic iron by factors of 10 to 100 using electrochemistry, substantially reducing the net first-cost barrier to using this technology.

We will develop a small-scale device that will be analyzed with electrochemical system models to permit performance prediction of full-scale devices. In addition, we will study the chemical reactions of interest in a synthetic ground water with the same composition, $\mathrm{pH}$, dissolved oxygen, and arsenic content of typical Bangladeshi ground water. This will allow us to understand how the reactions will behave in the unique Bangladeshi water environment, an area of study that has so far been neglected in the literature. The questions we will answer are related to the effects on the electrochemical system of the specific ionic species in the raw water, the $\mathrm{pH}$, dissolved oxygen and temperature effects on system performance, and the engineering design to make the system robust, fail safe, and most importantly, affordable to rural poor Bangladeshis.

\section{Accomplishments}

Several important achievements were made in FY06 that also laid the foundation for the continuing work in FY07. We successfully built a small-scale electrochemical device that allows us to vary the current density on the anode, and total charge applied to the cell, and which is ultimately scalable to a full size version. We compiled a realistic recipe for preparing in the lab (synthetic) Bangladeshi ground water that can be (and is) reliably reproduced in the lab on a regular basis. We developed a robust and reproducible protocol for measuring the final arsenic concentration, as well as the fractional speciation of As(III) to As(V), in a batch of synthetic Bangladeshi ground water that has been exposed to current in our small scale device. Finally, we established a proof of concept, verifying that exposure to a reasonable current in our small-scale device can reduce the final arsenic concentration to well below both the $50 \mathrm{ppb}$ and $10 \mathrm{ppb}$ health standards.

Our biggest accomplishment so far has been to measure a first-cut matrix of the final arsenic concentration in synthetic Bangladeshi ground water resulting from exposure to varying current density and total charge in a working small-scale electrochemical device. Guided by a goal of reducing the total arsenic content below the Bangladeshi standard of 50ppb (and if possible, below the WHO standard of 10ppb), we can use this matrix to determine the range of current density and total charge that warrants further investigation as we try to optimize these two parameters for all of the conditions found in Bangladesh. 


\section{Highly Efficient PLED Through Polymer Morphology Control}

PIs. Steve Johnson, Nitash Balsara, Jean Frechet, John Kerr, Gao Liu \& Rachel Segalman

\section{Project Description}

Major hurdle towards high efficient PLED (Polymer Light Emitting Diode) is that polymers can only convert singlet state excitons into photons. We propose to incorporate high efficiency phosphor dopant into a block copolymer system and use self-assembly to fabricate high efficiency, low manufacturing cost PLEDs. Block copolymers tend to microphase separate into regular, nanometer-sized domains. Using morphological controls such as annealing and blending, we will template the self-organization of the block copolymer into an ideal structure which will force the charge recombination to occur within the designed phase.

\section{Accomplishments}

Symmetrical (A) and non-symmetrical devices (B) were constructed using the new conductive block copolymer to evaluate its conductive behavior. The conductive polymer was spin-coated to form a nanostructured film (C). The I-V behaviors of the devices were shown in (D). Since the polymer forms microphase separated ET and HT domains, charges could be transported across the membrane using separate domains. The anode (ITO) and cathode (Gold) work functions are similar for (A), which primarily inject holes into the nanostructured conductive polymer. The forward and reversed biases (curve a in $\mathbf{D}$ ) are very similar for (A). This indicates the HT domains are uniform and continuous across the film. Device $\mathbf{B}$ has calcium as the cathode and ITO as the anode. Since electrons and holes can now be efficiently injected and transported from different domains, at forward bias, a significant higher current density (b in D) was observed compared to Device (A) at similar bias conditions. Under reversed bias conditions, device (B) shows negligible current flow due to the energy level mismatch between the electrodes and the conductive polymer. This confirms the true diode properties of the block copolymer.

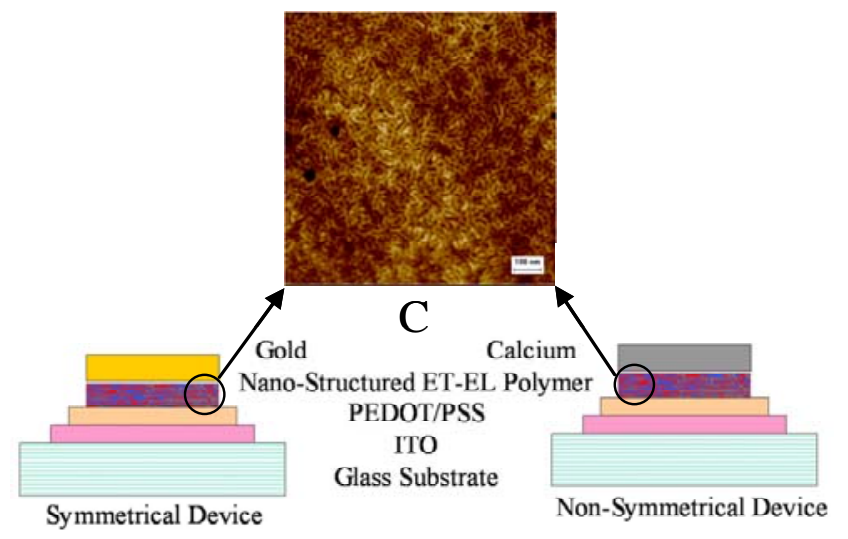

A

B

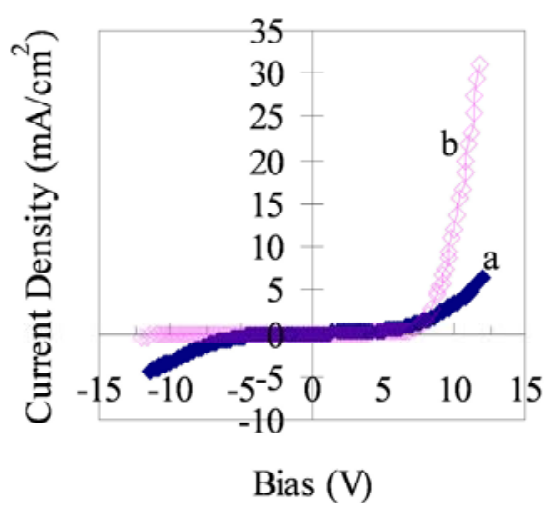

$\mathrm{D}$

Figure. A. A symmetrical device has gold and ITO as electrodes. B. A non-symmetrical device has gold and calcium as electrodes. C. AFM image of Nanostructure block copolymer thin film. D. I-V behaviors, curve a is from symmetrical device (The current density was $\mathrm{x} 3$ in order to show in the graph) A; Curve b, non-symmetrical $\mathbf{B}$. 
Surface Plasmon-Enhanced Photovoltaic Device

Principal Investigators: Robert Kostecki, Samuel Mao

\section{Project Description}

The purpose of this project is to develop and test an entirely different approach to produce a prototype of a photovoltaic (PV) device. This new concept offers an opportunity to create a completely new class of photovoltaic devices as compared to presently known PV systems. We will investigate whether intense photoemission of hot electrons from nanostructured thin-films can be used to design and develop a new class of plasmonic PV devices. We propose that photo-excited plasmons in a thin film nanostructured electrode discharge into hot electrons, which can be collected through a semiconductor Schottky diode during the steady illumination of the electrode with electromagnetic radiation. The electrons injected into the diode will be detected and measured as a short-circuit current

The project goals are to: (i) detect and quantify the plasmon-assisted electron emission at nano-patterned electrode/Schottky junctions, (ii) determine the effect of roughness of the electrode surface on the spectral range of excitation of SP's and the magnitude of photocurrent response, and (iii) build a prototype photovoltaic device which operates based on plasmon-enhanced photo-emission. We will also carry out studies of dynamic plasmon-assisted photoemission and use the results to develop deeper understanding of charge transfer mechanisms at or near the solid-solid interface. We plan to tune the roughness by adjusting the deposition parameters.

\section{Accomplishments}

Our primary accomplishment in the past year has been to develop a reproducible experimental protocol for preparing a thin-film photovoltaic device. Our methodology combines standard sputtering and deposition techniques. This procedure was intended to use a p-type $\mathrm{Si}(100)$ covered by $\mathrm{Si}_{2}$ insulating layer substrate and deposit various layers and a nano-structured electrode on top of the "sandwich- like" thin-film architecture. The deposition process was optimized to produce thin-films with a high concentration of imperfections to establish the proper energy barrier for the electrode/Shottky diode. The morphology of the electrode layer was tailored to a desirable particle size.

We have succeeded in manufacturing two early prototypes of the device. One of the prototypes displayed a weak (a few $\mathrm{nA}$ ) current for $\sim 1$ hour in response to electromagnetic radiation. These preliminary results indicate that the diode design and materials must be further optimized to improve the photon collection and charge separation efficiency. We are pursuing research to explore the key elements, which affect the mechanism of electron generation and flow during illumination of the electrode layer. 


\section{Analysis of High-Temperature Polymer-Electrolyte Fuel-Cell Phenomena Principal Investigator: John Newman}

\section{Project Description}

The purpose of this project is the examination of polymer-electrolyte fuel-cell (PEFC) behavior for possible operation at $120^{\circ} \mathrm{C}$. This temperature is above the maximum currently being used today $\left(\sim 80^{\circ} \mathrm{C}\right)$, and is thought to be key in the realization of the technology for transportation applications. While there is much research ongoing to find suitable individual materials and components that operate at $120^{\circ} \mathrm{C}$, there is a lack of understanding of how a PEFC operates at this higher temperature. For example, due to the much higher vapor pressure of water, the dilution effect of oxygen by the product water is expected to be more significant. Furthermore, though there is a gain in reaction kinetics, the open-circuit potential decreases with temperature, and hence there is a tradeoff between the useable energy that can be achieved by increasing the temperature. In addition, there are other, possibly unknown, tradeoffs such as the changes due to material properties like the conductivity of the membrane as a function of water content. Finally, the value of $120^{\circ} \mathrm{C}$ is chosen based on what is standard for current automobiles and not on optimization of a given PEFC system. It is the purpose of this project to examine these tradeoffs. Specifically, the main goals of the project are to understand, examine, and optimize the operation of a high-temperature PEFC through simulation.

In order to accomplish the goals, a numerical simulation will be developed. This involves a pseudo-two-dimensional macrohomogeneous model based on equations that describe the governing phenomena. This model will be validated against experimental results of cell polarization using experimentally measured parameters from the literature. Simulations will provide fundamental understanding and insight into the problems associated with hightemperature PEFC operation. Later, simulations will be used to examine the tradeoffs and effects of the various material properties such as conductivity, hydrophobicity, etc. These design criteria will provide realistic targets for experimental research and allow for high-temperature PEFCs to be developed.

\section{Accomplishments}

The most significant accomplishment is the development of mathematical code to describe PEFC operation at $120^{\circ} \mathrm{C}$. The 1-D and pseudo 2-D code has been written, tested, and debugged. The code contains the underlying physical phenomena including two-phase flow of liquid (water will form inside very hydrophilic pores) and vapor, detailed reaction-layer kinetics with microstructural information, and a robust description of water and proton movement in the membrane. The 2-D framework allows for examining different flow arrangements such as counterflow and coflow of the reactant gases.

The model has undergone some validation by fitting experimental polarization-curve data. This is done with a minimum number of fitting parameters, with the majority of the values coming from literature sources. The impact of water has been shown to be very important to consider even with dry feeds because many of the suggested membranes are not allowing any back-flux of water in the membrane, thereby greatly increasing its amount oon the cathde where it is generated. Furthermore, the cathode is limiting the system, especially in the use of air instead of pure oxygen.

Future plans include setting design targets and goals for a range of properties including conductivity and under various operating-condition scenarios. 


\section{Photons To Fuels - The Electrochemical Reduction Of Carbon Dioxide To Methanol Principal Investigator(S): Richard Russo and John Newman}

\section{Project description:}

This project, which is part of Helios, aims at developing a process for converting $\mathrm{CO}_{2}$ to methanol with electricity. To this end, two routes are being investigated. One is to make syngas (e.g. a mixture of $\mathrm{CO}$ and $\mathrm{H}_{2}$ ) by the simultaneous reduction of $\mathrm{CO}_{2}$ and $\mathrm{H}_{2} \mathrm{O}$ in a single electrochemical reactor (the syngas would subsequently be converted to methanol in a chemical reactor). The other is to make methanol from $\mathrm{CO}_{2}$ electrochemically through a combination of enzymatic catalysts attached to an electrode through nanowires.

Advantages of the first route are that technologies already developed for electrolyzers and fuel cells can be borrowed. Such a design allows for high current densities and a wide range of catalysts. Development of this system is also being supported with fundamental studies of the catalytic reaction mechanisms of $\mathrm{CO}_{2}$ and $\mathrm{H}_{2} \mathrm{O}$ in specially designed cells and with mathematical models based on macroscopic transport and kinetic properties .

Advantages of the second route include (1) minimal energy waste associated with "overpotentials" and (2) avoidance of "poisoning” of the catalysts as seen in traditional $\mathrm{CO}_{2}-$ reducing cells. Starting with $\mathrm{CO}_{2}$ and water, formate-, aldehyde-, and alcohol-dehydrogenase are used to reduce $\mathrm{CO}_{2}$ to formate, then to formaldehyde, and finally to methanol. To improve the rate and efficiency of electron transfer, chemical redox intermediaries will be replaced by conductive, biological nanowires (e.g., from Geobacter sulfurreducens) or conductive polymers that wire the enzyme's redox center directly to the electrode. This "wiring" of the enzymes will increase the local enzyme concentration and should enhance enzyme stability.

\section{Accomplishments:}

Electrochemical cell producing syngas $\left(\mathrm{H}_{2}+\mathrm{CO}\right)$ : An initial electrolysis cell was fabricated based on an extensive bibliographic survey from which the selection of the electrode catalysts and electrolyte were made. So far, this cell has been optimized by a "trial and error method," and is now capable of producing $\mathrm{CO}$ and $\mathrm{H}_{2}$ with faradaic efficiencies of $70-80 \%$ and 30-20 \%, respectively, at a total current density of $c a .25 \mathrm{~mA} / \mathrm{cm}^{2}$ over a10 h period. Confirmation of the successful conversion $\mathrm{CO}_{2}$ was supported with gas chromatography. In parallel, a conventional electrochemical cell was designed and built to provide product distribution data. These data are to be used as input for a mathematical model written to elucidate the kinetics of $\mathrm{CO}_{2}$ and $\mathrm{H}_{2} \mathrm{O}$ reduction for a chosen catalyst.

Electrochemical cell producing methanol based on enzymatic catalysis: Much of the initial effort was devoted to equipment fabrication, literature reviews, and methods development. An anaerobic, two-compartment chamber was fabricated to function both as a fuel cell and fuel generator and to produce nanowires. The ability to make useable nano-wires was demonstrated. G. sulfurreducens was cultivated on acetate, generating $\sim 0.005 \mathrm{~mA} / \mathrm{cm}^{2}$ from electrons donated from microbes to a graphite electrode via nanowires. In parallel, a chemical redox route was used to evaluate dehydrogenases. Two sources of diaphorases were compared, with the more active $(\sim 0.02 \mathrm{mmol} / \mathrm{g}$ s) being porcine heart diaphorase. Quantitative assays (both optical and NMR-based) were developed for each individual reaction in the reduction of $\mathrm{CO}_{2}$ to methanol, and each reaction rate was measured. The rate of formate production was the lowest $(0.125 \mathrm{mmol} / \mathrm{g} s)$; however, much more active formate dehydrogenases (up to $1.7 \mathrm{mmol} / \mathrm{g} . \mathrm{s}$ ) have been reported and are currently being isolated. 


\section{Long-Term Global Energy Demand and Carbon Emissions Scenarios}

Jayant Sathaye, Mark D. Levine, Lynn Price, Stephane de la Rue du Can, Michael

McNeil, Virginie Letschert, Shyam Menon, Phil Price, Jonathan Sinton, and Nan Zhou

\section{Project Description:}

The Intergovernmental Panel on Climate Change (IPCC) has developed a set of global scenarios that report on the anthropogenic greenhouse gas emissions from all sources up to the year 2100 by ten geographic regions. The scenarios were developed using a set of models that lacked a detailed representation of demand-side drivers and technologies. These scenarios provide the overall conceptual framework in which emissions of greenhouse gases and their impacts are analyzed.

The purpose of this project is to develop scenarios of energy demand by end-use for ten global regions consistent with one or more IPCC scenarios. Surprisingly, the global energy demand scenarios developed by IPCC, other intergovernmental organizations (e.g., UN, International Energy Agency (IEA)), international oil companies, and national governments are lacking in end-use detail. Most of the scenarios provide no more detail than total energy use by buildings, industry, and transport. Little if any of the underlying drivers (energy service demand, technology type, usage, saturation, efficiency) are included. As a result, it is virtually impossible to understand or interpret the scenarios in terms of current or future energy demand. The LDRD project will for the first time provide such information to the community that analyzes climate change, and also for the development of indicators of energy efficiency.

\section{Accomplishments:}

We disaggregated two scenarios by sector (buildings, industry, transportation, and agriculture) for 10 world regions. This information was finalized after review and provided to the IPCC WGIII chapter authors and is being used and published in the final draft of the Fourth Assessment

We designed a Global Energy Demand Model (GEM) and collected publiclyavailable data as well as that provided by collaborating research entities worldwide. We disaggregated each end-use sector at three different levels of detail, starting at the macro level and working to the third level that contains end-use technologies. Collection of Level 3 data for "marker" countries allows us to understand the possible energy consumption trends in the remaining countries in that region. In addition, level 3 allows for construction of detailed efficiency scenarios.

We completed Level 1 by integrating historical energy consumption, GDP, and population data from the IEA with the IPCC scenarios for all countries. We completed the process of designing and gathering data at Level 2 on critical drivers of energy demand in the residential buildings and industrial sector by region. We completed the structure for Level 3 for the buildings sector and have populated the model with data for China and have collected data for India. In addition, we completed a global model for projecting saturations and energy use for residential appliances that will link macroeconomic drivers to energy demand within GEM.

Our work on development of indicators at each of the three levels is being recognized by the IEA and the World Bank in their efforts to initiate activities for the development of indicators in Brazil, China, India, Mexico and South Africa. 


\section{Genomics Division}

LB06033

Computational and Experimental Testing of Methods for Binning Sequences from Metagenomic Studies

Principle Investigator: Jonathan A. Eisen

Project description

The purpose of this project is to improve upon our ability to perform metagenomic analysis (sequencing DNA directly from environmental samples). We have two main goals. First is the development of model metagenomic data sets where the organism source of the DNA is known. This will allow the testing of bioinformatics methods that attempt to assign sequence reads to organisms (generally known as binning) which is a critical step in metagenomic analysis. Our second goal is to develop new methods for performing binning, making use of the model data sets to test our methods against other methods that have been described.

Accomplishments

We are taking two approaches for the development of model metagenomic data set computational simulations and laboratory simulations. For the computational simulations we are generating simulated metagenomic data sets by (1) selecting sets of completed genomes and (2) randomly sampling the original sequence reads from those genome projects where the number of sequence reads from each organism is varied to create different ratios of mixes of organisms. We have generated twenty simulated data sets and are using them to test various binning algorithms since we know the "answer" regarding which reads map to which organisms. We also plan on creating a web site for the scientific community that will allow others to use these simulations for metagenomic methods testing.

For our laboratory simulations, which are just beginning, we have selected ten organisms which are easy to grow and which have their complete genomes determined. We will grow these species and then mix the cells in different ratios and then take the samples through a standard metagenomic sequencing protocol (DNA isolation, library construction, sequencing, etc). These data sets will then be used much like the simulations above but will include all the vagaries of working with real organisms and DNA and may be qualitatively different from the computational simulations.

Once the laboratory simulations are completed in the next few months we will then use both of the data sets to aid in our development of new computational methods for binning sequences. 


\title{
Metagenomics-Enabled Analysis of Termite Hindgut Microbiota for Biomass Conversion and Cleaner Energy
}

\author{
Principle Investigator: Philip Hugenholtz
}

\section{Project description}

Termites efficiently transform recalcitrant plant biomass, lignocellulose, to valuable sugars and fuels (hydrogen, methane) by exploiting the metabolic capabilities of microbial symbionts inhabiting their hindguts. An understanding of the enzymes and biochemical pathways used in the termite hindgut will therefore lead to more efficient strategies for converting biomass to fuels and chemicals. However, the mechanisms used to achieve these transformations remain elusive in the absence of molecular data. The purpose of this proposal is to reconstruct the metabolic pathways responsible for biomass conversion in termites using a metagenomic approach, paving the way for industrial applications such as biological ethanol and hydrogen production.

\section{Accomplishments}

We conducted a second field trip to Costa Rica in May 2006. The primary aim of this trip was to collect additional samples of the gut system's alkaline proctodeal segment 1 (P1) of the same termite species (Nasutitermes $\mathrm{sp}$.) for metagenomic analysis. We collected approximately 2000 termites, with whole P1 segments from 500 termites. This larger number of individuals was required due to the smaller size of the P1 segment. Cloning and sequencing of the P1 DNA will be conducted. Additional termite species were collected on the sampling trip, however, the $16 \mathrm{~S}$ survey was abandoned due to reduced funding.

A complete first draft of the P3 lumen fluid metagenomic analysis is now in hand and will be submitted to either Science or Nature by the end of calendar year 2006. We have refined our observations in preparation for publication. We now infer that approximately half of the hydrogenases are involved in sensing hydrogen, a key metabolic intermediate, and may allow the bacteria to move along hydrogen gradients in the hindgut. This type of hydrogenase has not been observed in nature previously. Both the Fibrobacter-like bacteria and treponeme spirochetes have been definitively linked with cellulose hydrolyzing enzymes through full sequencing of fosmids, and sequence composition based classification of metagenomic fragments. The treponemes are carrying out the bulk of the acetate production from hydrogen and carbon dioxide and overall are the dominant members of the P3 community. We have estimated that 250-300 species are present in the P3 section, and that only 10 species account for $\sim 50 \%$ of the biomass. 
Project Title: Development of Cost Effective Sequence-Based Technologies to Identify Genomic Alterations in Cancer

Principal Investigators: Len Pennacchio, Paul Spellman, Jan-Fang Cheng

Project Description

Studies of cancer development and progression have identified recurrent somatic chromosomal aberrations as a principle cause of disease. The technology capable of detecting these aberrations has been slow and costly and as a result the vast majority of such events remain unidentified. The purpose of this project has been to (1) develop a cost-effective, highthroughput DNA sequence based method to identify structural alterations in cancer cells, and (2) unravel the basis of cancer by identifying the resulting genes disrupted by these aberrations. The resulting data will serve as "proof of principle" for application to a larger set of tumors.

We will implement two sequencing technologies that will dramatically reduce costs associated with characterizing genome structure. The first technology is "DiTags", which allows paired ends represented by terminal 18 base pairs of large DNA pieces to be concatenated into a single clone for sequencing. The second technology is the sequencing method developed at 454, where resulting DNA sequences will be compared to the reference human genome to identify regions where chromosome fusions have occurred as evidence by the paired DiTag ends. Rearrangements will be searched for genes that show aberrant expression or structure and these genes will be biologically assayed for their roles in oncogenesis.

\section{Accomplishments}

Our most significant accomplishment has been to develop a reproducible procedure to construct DiTag libraries. First, we have modified a fosmid vector to enable the construction of DiTag libraries from large insert clones. Second, we have optimized all the experimental conditions and constructed several $40 \mathrm{~Kb}$ DiTag libraries. As a proof of principle, we have generated 2 runs of 454 sequencing data from a DiTag library derived from a breast cancer cell line, BT474. The 454 data represented $\sim 235 \mathrm{~K}$ pairs of 18 -bp sequences, which represent about $3 \mathrm{X}$ clone coverage of this cancer genome. We were able to map about $81 \%$ of these DiTag sequences to a unique genomic position, and $7 \%$ to multiple locations due to repetitive sequences. We were unable to map about $12 \%$ of the sequences, which suggests that the error rate of 454 data is much lower than the originally expected 1 per $100 \mathrm{bp}$. The analyses of the DiTag data have identified 13 translocations, 23 deletions, and 23 inversions, and mapped all the amplicons in the BT474 genome. A large fraction of these rearrangements and amplicons have been previously identified in independent experiments, which validates the DiTag approach as a fast and cost-effective way of detecting chromosomal aberrations in cancer genomes.

We have also succeeded in developing software to analyze a large number of DiTag sequences. The existing tools for finding sequence matches (e.g. BLAST, BLAT, and pattern match) are too slow and frequently produce false negative results with short sequences. To overcome this problem we have developed tools to convert the human genome to a suffix array library and search it for perfect 18-bp matches. With these tools we were able to position $235 \mathrm{~K}$ DiTag pairs to the human genome in a few hours with only $4 \mathrm{cpu}$. We continue to improve our scoring tools to utilize the repetitive sequences to support rearrangement data.

We are now applying this approach to study several cancer genomes simultaneously with the hope of establishing the foundation for a large-scale study. As an offshoot, this technology is being explored by the Joint Genome Institute to further cost effective genome assemblies. 


\section{Life Sciences Division}

LB04010

Molecular Microscopy and Tomography

Principle Investigator: Manfred Auer

Project Description:

The goal of this project was to establish a novel artifact-free EM sample

preparation and molecular EM imaging modalities, such as high-pressure freezing/freezesubstitution and electron tomography, respectively, and to apply these approaches to several eukaryotic and prokaryotic model systems. To compliment structural analysis, tag-based labeling approaches are being adapted and further developed to allow for correlative light and electron microscopy, allowing the high-resolution subcellular localization of proteins, as well as determination of the 3D architecture of macromolecular machines in their cellular context.

The central approach used involves high-pressure freezing sample preparation, followed by freeze-substitution and resin embedding, followed by sophisticated 3D imaging of thinly sectioned cells and tissues (EM tomography). Due to the limited penetration depth of electron microscopes, thin sectioning of resin-embedded samples is essential for the study of tissues, microbes and microbial communities (biofilms). Complex 3D volumes were then examined by sophisticated interactive 3D visualization tools (some of which needed to be developed), which now allow fast interactive exploration, segmentation and quantitative analysis of tomographic volumes. Since not all proteins can be discriminated by shape alone, we have begun to explore three tagbased labeling approaches such as the tetracysteine/ReAsH/photoconversion, SNAPtag/photoconversion and the hexahistidine/Ni-NTA-nanogold systems. All these tagbased labeling approaches are still in an experimental stage, and have not been robustly adapted to electron microscopy detection.

Accomplishments:

We have successfully applied artifact-free sample preparation to a variety of specimens, including inner ear hair cells, kidney podocytes, premalignant and malignant breast cancer 3D cell culture systems, plant seedlings, as well as a variety of soil bacteria of high relevance to DOE-GTL, including Myxococcus, Shewanella, and Desulfovibrio. Tomographic reconstructions of bacterial colonies as well as mitochondrial ReAsH labeling, served as preliminary results for the successful GTL-PCAP grant application (PI: Mark Biggin). Additional results on plants and microbes will be used for the Biofuel grant application (PI: Jay Keasling). Our collaboration on breast cancer cells is reflected in several grant proposals submitted by Mina Bissell, including a Tumor Microenvironment Network U54 grant application.

We have elucidated the molecular 3D architecture of the hair cell mechanoelectrical transduction machinery, and a manuscript has been submitted. We are also the first to succeed in visualizing by EM lipid rafts and are currently preparing a manuscript for publication. On the kidney project we have obtained unprecedented preservation and are now in the process of 3D data collection and analysis. For bacterial biofilms we have obtained tomographic reconstructions that reveal the abundance of extracellular vesicles of defined structure, including intervesicular tethers, that are likely to play an important role in interbacterial material transfer and also signaling. We are currently preparing a manuscript on the biofilm architecture, and are in the final stages of analyzing the vesicle composition, which is likely to result in a publication in the very near future. 


\author{
Modeling Human Disease Genes In Drosophila Melanogaster \\ Principal Investigator: Susan Celniker
}

\title{
Project Description
}

Many of the genes that are deregulated by genomic aberrations in human cancers are conserved between Drosophila and humans. Our goal is to develop Drosophila-based analyses of human epithelial cancer gene functions by (a) designing strategies to efficiently generate strains in which candidate genes are inactivated or ectopically over-expressed (b) identifying functionally important genes in embryos using automated techniques to assess growth, proliferation, apoptosis and pattern formation in gain-of-function and loss-of-function mutants; and (c) locating candidate genes within specific pathways (epistasis analysis) for which morphological readouts exist using semi-automated approaches to assess adult fly reporter phenotypes. Specifically we plan to apply high throughput technologies and the power of Drosophila genetics to dissect cancer gene function and identify new components of cancer pathways.

\section{Accomplishments}

We focused on genes in regions that undergo high levels of amplification in patients with short survival ovarian cancer and with breast cancer near the ErbB2 amplicon. We have identified cognates for ten genes. We successfully generated RNAi transgenics driven by a UAS promoter to produce conditional loss-of-function mutations for seven of the ten genes. One gene, a cell cycle kinase called CRK7, when knocked out in wing tissue produces a curled wing phenotype presumably due to cell death and is lethal when knocked out early in embryonic development. We also identified P-element transposons inserted near the 5 ' end of five of the ten genes. Excisions of the transposons potentially produce loss-of-function mutations. Ten excisions of the P-element near $C R K 7$ are homozygous lethal. Characterization of the excisions is underway. In order to assay the effect of gene overexpression, we made UAS transgenics for six of the ten lines. Overexpression of $C R K 7$ in wing tissue causes a similar wing phenotype to that seen in the loss of function mutant.

We found that Breast carcinoma amplified sequence 4 (BCAS-4) is similar to the human gene cappuccino, a member of the BLOC-1 complex required for normal vesicle trafficking to lysosome. The process of endocytosis has recently been linked to the control of cell proliferation and defects in endocytosis to a role in neoplasia. To investigate the role of endocytosis in cancers, we made RNAi lines for one cell polarity gene and 11 Drosophila cognates of genes in the endocytosis pathway. Our collaborators in David Bilder's lab (UCB), experts in studying neoplastic tumors in Drosophila, have used these transgenic flies to generate endocytosis-specific phenotypes for ten of the 11 endocytic genes, including the Rab protein Syntaxin 13, Rab effectors, rabaptin and rabenosyn, co-factors Slyl and vps 45, multivesicular body (MVB) escort II protein, vps25, and escort III proteins, vps2, vps20, vps 24, and vps32. We also made lethal excisions for five genes, vps32, vps36, vps 45, rab-4 and Slyl. Lethal excisions of vps45 and vps32 generate a neoplastic tumor suppressor phenotype, rab-4 and Slyl die but are not transformed and vps36 is in the process of being analyzed.

RNAi is extremely powerful but results are complicated by the differential expression of transgenes caused by position effects at different insertion sites. To minimize position effects we have developed a new Drosophila RNAi vector for transgenics that contains the site-specific recombination element, attB, allowing targeted integration. In collaboration with Michele Markstein in Norbert Perrimon's lab, we screened over 40 attP lines to find the best genomic environment for our studies.

Finally, in related work, in collaboration with Jian Jin's LBNL engineering group, we did preliminary work toward building automated systems. Our microinjection robot generates trangenics at $50-75 \%$ the efficiency of a skilled human operator while performing injections as fast or faster. Another system now, proceeding with follow-on funding, is for automated phenotype identification and fly sorting. 


\section{Unmasking the Human Genome Alternative Splicing Program Principal Investigator: John Conboy}

\section{Project Description}

Alternative pre-mRNA splicing is a major mechanism for regulation of gene expression in the human genome, allowing a modest number of genes to encode a highly complex proteome. Here we are employing high resolution microarray technologies for quantitating mRNA expression with single exon resolution, facilitating analysis of alternative splicing in normal development, and how aberrations in the process contribute to human disease including cancer. The major goals of this project are (1) to use novel exon microarrays and bioinformatics tools to illuminate genome-wide, tissue-specific patterns of alternative splicing; (2) to identify evolutionarily conserved regulatory sequences that interact with the splicing machinery to orchestrate the human genome alternative splicing program; and (3) to study causes and consequences of aberrant splicing in human cancer.

\section{Accomplishments}

During the second and final year of this LDRD study, we extended our analysis of programmed alternative splicing to additional differentiated cell types. This collaboration with Affymetrix scientists employed their new exon microarray format and facilitated identification of the first substantial dataset of muscle-specific exon, a critical first step in understanding the muscle splicing program. Our major finding is that a functionally coherent group of cytoskeletalrelated genes appears to be coordinately regulated by a set regulatory sequence motifs in the flanking introns. A draft manuscript describing these studies in collaboration with Inna Dubchak, Debopriya Das, and our Affymetrix collaborators is to be submitted imminently.

To validate computational results, we are performing experimental analysis of the muscle splicing program in a zebrafish model system (collaboration with Sharon Amacher at UC Berkeley). Preliminary results show that knocking down expression of a key splicing factor in zebrafish embryos alters regulation of target exons having nearby binding sites for that splicing factor, supporting one of our major computational predictions.

In studies supported by a new NIH / R21 proposal, we have also initiated studies using the exon arrays to explore programmed alternative splicing in erythropoiesis.

Finally, we are also using the Affymetrix exon arrays to explore disruption of normal splicing programs that is characteristic of human cancer. Breast cancer studies are being pursued in collaboration with Life Sciences Division Director Joe Gray, and the Affymetrix team; recently we have validated several abnormal splicing events in a panel of representative cell lines. Breast, brain, lung, and ovarian cancers will be profiled in collaboration with two recently funded programs, the Berkeley Cancer Genome Center and the Clincal Proteomic Technologies for Cancer enterprise. The long range goal of these analyses is to determine the incidence and biological importance of aberrant pre-mRNA splicing in tumor biology.

In conclusion, the results of this LDRD thus far support the hypothesis that highly coordinated programs of alternative pre-mRNA splicing can be elucidated through the use of exon microarrays, This strategy may provide fundamentally new insights into many biological systems, including several of interest to other Berkeley Lab programs, and facilitate new mechanistic studies of the splicing regulatory networks that control tissue-specific splicing. These new insights may ultimately suggest therapeutic approaches to aberrant splicing in human cancer. 
Improved Phase Contrast for Cryo-EM of Biological Machines and Subcellular Structure Principal Investigators: Robert Glaeser, Jian Jin, Kenneth Downing, Eva Nogales, Manfred Auer

\section{Project Description}

The goal of this project is to obtain preliminary results that establish that a microfabricated electrostatic device can selectively apply a 90 degree phase shift to the central (unscattered) beam in the back focal plane of the objective lens of an electron microscope. This phase-shift device will provide "ideal" phase contrast for in-focus electron microscopy of biological specimens. The concept of using microscopic electrodes to build an electrostatic phase-shifting element would have been impractical without the development of micrometer-scale threedimensional fabrication techniques, and, even then, before the more recent development of electron-optical correctors. Significant engineering challenges were (1) to prevent contamination of, and subsequent electrostatic charging of, the phase-plate device and (2) to keep it centered on the unscattered electron beam.

The short-term need is to acquire sufficient preliminary results on which to base strong proposals for long-term grant funding in the areas of single-particle cryo-EM and EM tomography. The long-term opportunity, if this project is successful, will be to achieve many of the ambitions articulated in the DOE statement of goals within the areas of macromolecular and cellular imaging.

Accomplishments

A novel, 2-electrode device was investigated as a possible phase-contrast aperture for the electron microscope. This design was chosen because of the high likelihood that it could be fabricated much more easily than the more conventional, 3-electrode einzel lens. Electrostatic calculations of the "fringing fields" were also used to show that deviation of the 2-electrode device from ideal performance would be very small, and considerably smaller than that of the 3electrode einzel lens.

Experiments were then carried out with a prototype device that was fabricated with smallest feature sizes of 2 micrometers. A custom-built positioning device was also designed and fabricated at LBNL, which allowed accurate centering of the aperture as well as the application of the desired voltage to the central electrode of the device. These experiments used the voltagedependent movement of maxima and minima in the contrast transfer function of the electron microscope to demonstrate that the desired 90-degree phase shift was, indeed, provided by this device.

A paper describing the 2-electrode design, electrostatic calculations of its fringing fields, details of its microfabrication, and experimental proof-of-concept that the device provides the expected phase shift was accepted for publication. Additional image-calculations, not yet published, have been completed, which provide guidance on the combination of device-size and objective lens focal length that are required in order to achieve use of the phase-contrast aperture. On the basis of work completed in FY 2006, we have achieved our goal to obtain the full amount of preliminary results that are needed in order to seek follow-on funding for this project. 


\section{Neuroimaging with Advanced Molecular Probes \\ Principal Investigator: William Jagust}

\section{Project Description}

The focus of this project is to develop methods for the non-invasive quantitation of the dopamine system and methods for quantitating the development of brain amyloid. Our investigations of the dopamine system have utilized the positron emission tomography (PET) tracer fluorometatyrosine, (FMT), along with functional magnetic resonance imaging (fMRI) to measure brain blood flow in response to cognitive activity. We have thus studied a group of older individuals in an effort at investigating relationships between declining dopamine function, brain physiology, and aging in order to probe mechanisms of compensation in the aging brain.

With regard to the amyloid studies, we have successfully deployed the tracer 11C"Pittsburgh compound B" or PIB in order to investigate binding properties and quantitative methods for detecting amyloid in the brains of living humans. Amyloid is the protein deposited in the plaque in Alzheimer's disease (AD). The goal of this project was to investigate the detection of amyloid in normal older people and those with dementia. In particular, we hypothesized that individuals with Frontotemporal Lobar degeneration (FTLD), a non-amyloid dementia, would have negative PIB scans and be differentiated from AD patients with amyloid imaging.

\section{Accomplishments}

We completed 22 studies in older individuals (over age 50) and 11 studies in younger individuals, with FMT-PET and fMRI measures of brain activation. We perfected techniques for acquiring and quantitating these data. Preliminary analyses in the older individuals show that those with higher FMT uptake (higher dopamine synthesis capacity) show better performance on working memory tasks, confirming our initial hypothesis. We have also found, with fMRI, that while subjects perform working memory tasks frontal lobe regions become active and that the higher the dopamine synthesis, the greater the brain activity. Together, these results show that declining dopamine levels are related to reduced ability to activate the brain during working memory, and that these factors are related to poorer working memory performance. These results have major implications for brain aging. In a follow up NIH project we will be investigating these changes in comparison to function in normal younger people.

In the interest of examining the clinical utility of amyloid imaging with PIB, we studied patients meeting research criteria for $\mathrm{AD}(\mathrm{N}=7), \operatorname{FTLD}(\mathrm{N}=12)$ and cognitively normal controls $(\mathrm{N}=8)$. PIB whole brain and region of interest (ROI) Distribution Volume Ratios (DVR) were calculated using Logan graphical analysis with cerebellum as a reference region. DVR images were visually rated by a blinded investigator as positive or negative for cortical $11 \mathrm{C}-\mathrm{PIB}$, and summed $18 \mathrm{~F}-\mathrm{FDG}$ images were rated as consistent with AD or FTLD. All AD patients (7/7) had positive 11C-PIB scans by visual inspection, while 8/12 FTLD patients and 7/8 controls had negative scans. Of the four PIB-positive FTLD patients, two had 18F-FDG scans that suggested AD, and two had 18F-FDG scans suggestive of FTLD. Mean DVRs were higher in AD than in FTLD in whole brain, lateral frontal, precuneus, and lateral temporal cortex ( $\mathrm{p}<$ 0.05 ), while DVRs in FTLD did not significantly differ from controls. These results confirm our hypotheses that imaging with PIB) helps discriminate Alzheimer disease (AD) from frontotemporal lobar degeneration (FTLD). In a follow-up NIH funded project we will investigate a larger number of subjects with autopsy correlations. 


\section{Determine if PIR51 is a Potential Tumor Suppressor Gene Similar to BRCA2 Principal Investigator: David Schild}

\section{Project Description}

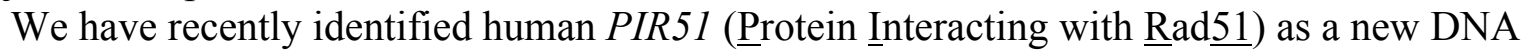
repair gene. Our goal is to further characterize PIR51 and to obtain experimental data (i.e. "proof of principle") to test our hypothesis that this gene functions in the same pathway as $B R C A 2$, a tumor suppressor gene important in breast cancer. Partial depletion of the Pir51 protein by RNA interference (RNAi) sensitizes HeLa cells to a DNA cross-linking agent and to camptothecin (a Topol inhibitor), and causes a high frequency of chromatid breaks following DNA damage. Therefore, cells partially depleted for Pir51 share some properties with BRCA2defective cells, but differences do exist. These differences may be artifacts due to the incomplete depletion of Pir51 or to the use of HeLa cells. Our aim is to test the phenotype of: 1) stable depletion of Pir51 in near-normal human cells, and 2) a pir51 knockout in DT40 cells. We will also test if PIR51 functions in homologous recombinational repair (HRR), as does BRCA2.

Our short hairpin RNAs that target Pir51 expression will be subcloned into a retroviral vector and will be used to infect two near-normal human cell lines (hTERT-immortalized fibroblasts and hTERT-mammary epithelial cells). The phenotype of these cell lines after Pir51 depletion will be tested, and compared to our results for HeLa cells. A recombinational-reporter system will be used to test if Pir51 is involved in HRR. A pir51 knockout will be isolated in the chicken DT40 model system. Phenotypic characterization of a DT40 pir51 knockout will test if it shares common traits with knockouts or partial deletions of other genes, including brca2. A second pir51 knockout will be isolated in a brca2-defective DT40 cell line in order to test for epistasis of PIR51 and BRCA2.

\section{Accomplishments}

Our major accomplishment was to determine that BRCA2 and Pir51 share several characteristics in common. Like BRCA2, the Pir51 protein had been reported to directly interact with the RAD51 protein. We have confirmed this interaction and mapped the region of Pir51 that interacts with RAD51. This RAD51-interacting region was mapped to the C-terminal 25 amino acids of Pir51, now referred to as Pir51-CTD (C-terminal domain). This result has recently been published in Nucleic Acids Research, and the LDRD support acknowledged. We have also determined that Pir51, like BRCA2, is directly involved in homologous recombinational repair. Using a recombinational reporter system integrated into a human lymphoblastoid cell line, we have shown that depletion of Pir51 results in a significant decrease in recombination. This result is part of a manuscript on Pir51 that we have recently submitted.

In order to better suppress the level of Pir51 in different human cell lines, two different retroviral constructs expressing shRNAs targeting Pir51 (for depleting Pir51) have been made, as has an additional construct expressing a control hairpin (for negative control).

Progress has been made towards generating a pir51 knockout in DT40 cells. Vectors for making the plasmid constructs have been obtained, DT40 cells have been cultured, and genomic DNA has been isolated and used to PCR amplify both the 5-prime and 3-prime regions for subcloning into these vectors. Gene targeting constructs have been made and are currently being used to knockout the first of two Pir51 alleles in DT40 cells. 


\section{Expression profiling of radiation and cancer susceptibility genes \\ Principal Investigator(s): Andrew J. Wyrobek}

\section{Project Description}

The long-term objectives of the proposed research are to identify genes and pathways that predict individual susceptibilities to radiation-induced DNA damage and cancers. This project will address the feasibility of using gene-expression profiles and measurements of early DNA damage responses to predict subsequent health risks. Anticipated findings will identify candidate gene and pathway for future screening of individuals for abnormally clinical sensitivities to radiotherapy and to develop drug strategies to reduce radiation DNA damage to healthy tissues (radioprotection) and reduce cancer risks (prevention).

We will develop a mouse model of radiation sensitive genes that control cellular damage after exposures at low dose and low dose rates. It is known that tissues and cell types within tissues differ dramatically in their sensitivity to ionizing radiation at high doses, but little is known about the genetic and physiological factors that control susceptibility, especially for low doses. Under certain circumstances, exposure to low doses of radiation confers protection against subsequent DNA damage, chromosome damage and cancer, a phenomenon known as radioadaptation. Our project will investigate the time course of early DNA repair and gene expression profiles after radioadaptive exposure regimens and evaluate their utility for predicting subsequent chromosomal damage and ultimately, cancer risk. We will utilize various genetic mouse models including those with mutated $\mathrm{Tp} 53$, a key controller of the cellular response to ionizing radiation and modulator of cancer risk.

This project will elucidate the molecular determinants of the low-dose radioadaptive response in vivo in mammals. This gene- and pathway-specific knowledge of the early cellular responses to low-dose ionizing radiation will help to reduce the uncertainty of assessing potential tissue damage and health risks after whole body exposures to low-dose ionizing radiation.

Accomplishments:

We completed a detailed survey of radiation-responsive proteins in mammals and it was published in the International Journal of Radiation Biology in November 2006. This review identified $\sim 260$ genes whose cellular expressions were modulated after radiation exposure. A subset of genes showed post-exposure time and dose response characteristics that are promising for individual radiation biodosimetry.

We initiated a collaboration with colleagues at UCSF for mice with various types of Tp53 mutations that are known to have different sensitivities to radiation-induced cancer. We plan to use these mice to identify the early cellular and gene-expression responses that protect against radiation-induced chromosome damage and cancer. We are currently developing protocols for repeated small samples of mouse cells for measuring DNA repair kinetics (by single-cell-gelelectrophoresis), nuclear DNA damage foci (by gH2AX, pATM, 53BP1), gene expression profiling (by genome-wide approaches), residual chromosomal damage and cancer risk. Studies are being performed in mice with differing cancer-prone genotypes and in different radiationsensitive tissues to determine the association between DNA damage response and expression profiling in peripheral blood and the damage that occurs in cancer-susceptible tissues. 


\title{
Materials Sciences Division
}

\section{LB06002}

\author{
Electron Microscopy of Soft Matter \\ Principal Investigators: Nitash Balsara, Ken Downing, Jay Groves, Christian Kisielowski, \\ Andrew Minor, Dieter Typke
}

\section{Project Description}

Important scientific challenges such as understanding the structure and function of biological cell membranes and developing new polymers for fuel cells require characterization of soft matter on nanometer length scales. Our objective is to study such systems by electron microscopy. High resolution electron microscopy at NCEM has provided crucial insight into a variety of hard materials including metals, semiconductors, and ceramics. We will build on this strength and launch a program that examines both biological and synthetic soft matter in new and unique ways.

\section{Accomplishments}

The group has developed a phase plate for the LIBRA 200FE electron microscope, which consists of a piezoelectrically driven objective aperture, control electronics and a lithographically fabricated electrostatic mini-lens. Tomography series of soft materials recorded in STEM and TEM modes were reconstructed utilizing typical back projection schemes and compared with results obtained by application of discrete tomography. Noise reduction and contrast enhancement were demonstrated.

We have made progress in using existing electron microscopy facilities for imaging soft matter. Electron tomograms of lipid vesicles containing membrane proteins have been obtained. In the absence of the membrane proteins, the vesicles are perfectly spherical. The addition of the membrane protein leads to ellipsoidal vesicles. Understanding the role of proteins on the curvature of the lipid membrane is important for elucidating the mechanism of intercellular signaling and membrane binding events. Electron micrographs of lithiumcontaining block copolymers have been obtained by spatially resolved energy loss spectroscopy. We demonstrated that the lithium ions are clustered and localized toward the middle of the lamellae. To our knowledge, this is the first report of imaging lithium with nanoscale resolution using energy filtered microscopy. The first version of the phase plate described above will work over a limited frequency range of 1-5 $\mathrm{nm}^{-1}$. We have synthesized block copolymers with $4 \mathrm{~nm}$ periodicity for testing the phase plate. These materials are also of interest for proton conduction in fuel cells. We expect that water will be retained in these channels above the normal boiling point of water due to capillary condensation. Tomographical reconstruction and energy filtered imaging of these soft materials has led to unique understanding of the nanostructure and will eventually provides us with unprecedented structure-property relationships.

In the post-LDRD period, additional testing and enhancements of the technologies and system are underway. 


\section{Technique Development for Aberration Corrected Microscopy at NCEM Principal Investigator: Ulrich Dahmen}

\section{Project Description}

The field of electron microscopy is undergoing a revolution based on major recent advances in electron optics, detectors, stage design and computing power. These advances make it possible to overcome the fundamental limitations currently imposed by lens aberrations and the TEAM construction project, led by NCEM on behalf of five DOE microscopy efforts (LBNL, ANL, BNL, ORNL and FS-MRL at UIUC) will initiate a new generation of electron optical instrumentation. However, in the process of surpassing traditional instrumentation limits, new barriers often rise to the surface.

The current project will address some basic scientific and technological issues to help lay the scientific foundation and prepare for the challenges posed by this new technology. The objective is to develop techniques for imaging, spectroscopy, samples, stages, data analysis and operating parameters under aberration-corrected electron microscopy. To take advantage of the greatly increased sensitivity of aberration corrected beams it will be necessary to develop new sample preparation methods. It is equally important to begin the evaluation of prototype aberration correctors and monochromators, in close collaboration with their designers, in the context of scientific research on the atomic structure of materials.

\section{Accomplishments}

Following thorough acceptance tests, NCEM's VG STEM microscope, retrofitted with an

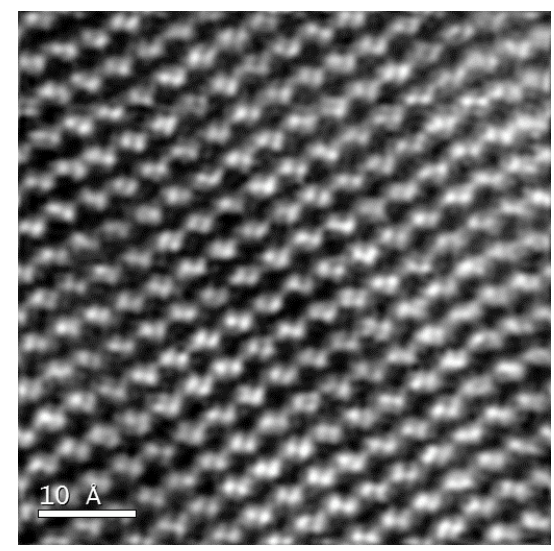

Figure 1. HAADF image of GaAs in [110] orientation obtained on the aberration corrected VG HB501. The 1.4 angstrom dumbbell is clearlv resolved aberration corrector, was moved to a newly-refurbished laboratory. The aberration-corrected VG machine is now fully operational, with spatial resolution better than 1.4 angstrom routinely attained at $100 \mathrm{kV}$ (see Fig. 1). The high resolution capabilities of this machine have already attracted several scientific projects, including exciting results on the interstitial nature of $\mathrm{N}$ doping in GaAsN quantum wells, which are currently being processed for publication. Other research highlights include structural analyses of $\mathrm{CeO}_{2}$ nano-bundles and $\mathrm{Na}_{8} \mathrm{Si}_{46}$ clathrate compounds, and lattice relaxation studies of FePt nano-particles.

In addition, the VG STEM is proving the ideal platform for TEAM personnel to get acquainted with the practical aspects of aberration-corrected microscopy, more specifically to familiarize themselves with the techniques for measurement and correction of the aberrations. The diagnosis of the aberrations on the TEAM instrument will rely on so-called "Zemlin tableaux", comprising a series of diffractograms of an amorphous region of the sample, taken at different beam tilts. The distortions observed in the diffraction rings as a function of tilt angle can be interpreted to yield precise measurements of the aberrations. The main drawback of this fully automated procedure is its need for an amorphous area, which implies that the customary sample plasma cleaning procedure is inadequate. Plasma cleaners destroy most carbon-based materials on the sample, which are generally needed to measure aberrations and tune the microscope. Based on this analysis, alternative sample cleaning facilities, such as mild heat treatment in a vacuum chamber, will therefore be put in place at NCEM. Furthermore, an independent algorithm to fine-tune the column directly on the crystalline area of interest in STEM mode was shown to be applicable to the case of TEAM. Practical tests of the potential of this recently-developed "achromatic tuning method", which relies on the interpretation of focus-independent contrast in convergent beam diffraction patterns, were carried out by collaborators at BNL. 
Tailoring the Self Assembly of Functionalized Biomolecular Building Blocks

Principal Investigators: Matthew B. Francis, Phillip L. Geissler, and James J. De Yoreo

\section{Project Description}

Self-assembling biomolecules could provide powerful and efficient scaffolds for the construction of new materials. In addition to possessing nanoscale dimensions overall, the structures they produce often display periodic features that could position functional objects with ultrahigh resolution. However, most scaffolds reported to date can only assemble into a limited number of geometric arrangements, and little is known about the long range order that these structures can establish. To explore these issues, a systematic and multidisciplined approach is being used to elucidate the fundamental mechanisms that govern biomolecular self assembly on multiple length scales. These aspects will be explored through the development of a versatile viral capsid-based system for the preparation of ordered 2-dimensional materials.

These studies will yield new techniques for nanoscale patterning, as well as fundamental insight into the thermodynamic and kinetic parameters required to design entirely synthetic selfassembling systems. As an initial experimental target, dynamic systems capable of mimicking biological light harvesting systems are being sought.

\section{Accomplishments}

In the first year of funding, a mutant of the tobacco mosaic virus (TMV) coat protein was expressed in bacteria. Disk structures assembled from this protein display multiple copies of a uniquely reactive cysteine residue on a single surface. These sites have been modified, resulting in the introduction of 17 disk structures on top of the disks. We have found that the disks remain assembled after the modification, and in separate studies we have found that they retain functionality.

To attach the disks to patterned surfaces, we have developed the selective chemistry to modify the "bottom" surface and characterized the interactions of the resulting disks with mica substrates using atomic force microscopy. In current experiments, substrate patterns are being used to orient the disks, and new linkages are being used to allow movement in the X-Y plane.

We have also developed a simple computational model for disk assembly on solid substrates, and we have performed exploratory simulations to characterize its range of behavior. The model represents functionalized TMV capsids as hard disks in two dimensions, endowed with short-ranged, orientationally specific interactions. Synthetic groups that possess these interaction capabilities can indeed be introduced on disk periphery using synthetic approaches. Kinetic Monte Carlo algorithms advance periodically replicated systems of these disks efficiently and with dynamical realism. By varying the number and strength of the peripheral interactions, vapor-, liquid-, and solid-like phases have been identified. More exotic phases can be generated simply by limiting the extent of chemical modification. These simulations have revealed a pragmatic strategy for assembling disks into biomimetic patterns. In combination with the synthetic advances described above, this theoretical model is now being applied to build multicomponent biological systems. 


\section{Light-activated ion channels and transporters Principal Investigators: Ehud Isacoff, Dirk Trauner, Richard Kramer.}

\section{Project Description}

Ion channels and transporters are proteins in the lipid membranes of all cells whose activity is controlled by voltage, chemicals, temperature, and mechanical forces. Our Strategic LDRD proposed to create nanoscale phototransistors by modifying these ion transport proteins with novel chemical moieties that act as photo-activated "gates." The ability to switch ion transport elements on and off remotely will be complemented by the incorporation of optical reports of function that can be used to couple elements in artificial membranes to opto-electronic circuits.

\section{Accomplishments}

As described in last year's report, our first activities focused on constructing a lightactivated non-selective cation channel based on Synthetic Photoisomerizable Azzobenzene

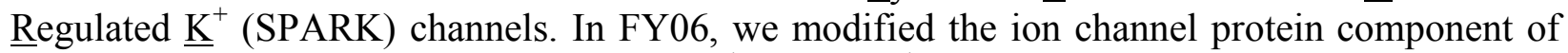
SPARK channels to lose selectivity of $\mathrm{K}^{+}$over $\mathrm{Na}^{+}$, thus creating excitatory, rather than inhibitory, light-gated channels (Chambers et al., 2006).

In FY06, the main focus was on light-gated glutamate receptors. With proof of principle at hand that $\mathrm{K}^{+}$channels can be engineered to become sensitive to light, we propose to extend to ligand-gated ion channels, such as glutamate receptor/channels. Recent work by the Gouaux and Mayer groups provides a detailed three-dimensional picture of the binding domain of glutamate receptor/channels (iGluRs). We synthesized a "glutamate on a photoisomerizable string" that can be tethered to an introduced cysteine near the binding pocket on the large extracellular ligand binding domains of glutamate receptors. The first of these has worked to reversibly gate in response to light the iGluR6 receptor, a channel that is permeant to both $\mathrm{Na}+$ and $\mathrm{Ca}^{++}$(Volgraf et al., 2006a). The light response is robust and the biophysical properties have been characterized at length (Gorostiza et al., 2006). This receptor endows neurons with the ability to be rapidly, accurately and reproducibly activated by light (Szobota et al., 2006).

As a complementary approach, we have made an untethered photochromic glutamate that can be used to reversibly switch between active and inactive forms and thus drive neuronal activity (Volgraf et al., 2006b).

Considerable progress was made in our aims. This brings us significantly closer to our goal of creating light-switched ion channels and transporters, which can serve as nanodevices and operate as optically controlled elements of nano-circuits. We have been recently awarded funding from the NIH Roadmap Initiative to create the Nanomedicine Development Center in Optical Control of Biological Function. This is one of 8 centers nationwide, and includes 8 Berkeley-LBNL faculty including the 3 PIs and 5 from Stanford, Caltech and Scripps. 


\section{Biogeochemical Reaction Rates and Pathways in Porous Media Principal Investigator: Ali Javey}

\section{Project Description}

In this project, we will explore integration of cheap and high performance electronic circuitry based on synthetic nanostructured channel materials, such as simiconductor nanowires, on bendable and wearable plastic substrates that are capable of operating in the ultra high frequency (UHF) regime (0.3-3 GHz). The ultimate goal is to demonstrate RF-ID tags based on nanowire thin-film transistors (TFTs).

As background, low cost, flexible, and high performance electronics are highly desirable as they enable a wide range of new and exciting consumer electronic applications, including flexible displays, RF-ID tags, bendable radars and antennas, and artificial skin. Conventional silicon CMOS technology is not compatible with the flexible plastic substrates since their processing temperatures are higher than the deformation temperature of plastics. Organic materials and amorphous silicon are the two main materials that have been actively explored over the past few years for flexible electronic applications, mainly owing to their low processing temperatures and their low cost of integration. However, they lack robustness and air stability, and are poor semiconductors, so therefore result in low performance electronics that degrade quickly over time. Recently, nanowire devices have been shown to operate at $\sim 10 \mathrm{MHz}$ on plastics. Through materials, device structure, and circuit design innovation and optimization, we plan to further enhance the performance by $\sim 2$ orders of magnitude in order to achieve truly high performance and flexible circuitry.

\section{Accomplishments}

This project was initiated late in FY06 to begin preliminary work with a commitment to continue into FY07. The first and major task was doing lab renovation necessary to conduct the research. We also started work on building some of our synthesis tools, especially the chemical vapor deposition (CVD) system required for the synthesis of nanowires. This CVD system is now operational and we are now synthesizing the nanowires that we need for our device/circuit applications. 
Terahertz-Frequency Conductivity and Ultrafast Optical Excitations in Single-Walled Carbon Nanotubes

Principal Investigator(s): Robert A. Kaindl, Yingzhong Ma, Daniel S. Chemla

\section{Project Description}

The goal of this LDRD is to study terahertz-frequency carrier transport and ultrafast electronic processes in single-walled carbon nanotubes (SWNTs). Terahertz (THz) and midinfrared (mid-IR) studies can reveal basic properties, such as plasma frequency, scattering rates, and gaps in metallic nanotubes, and can illuminate the influence of excitonic correlations and screening on the optical resonances of semiconducting SWNTs. Moreover, the relative amounts of homogeneous and inhomogeneous broadening of near-IR absorption lines can be investigated by following the irreversible decay of coherent polarizations. This will provide insight into the role of scattering with electrons, phonons, or excitons, and of higher order processes on the optical response. The insight gained here is critical for optoelectronic nanotube applications, e.g. for fast switches or novel light detectors and emitters at infrared wavelengths.

An array of ultrafast experimental techniques in a broad wavelength range is available to be used in these novel experiments: (i) High frequency transport properties and low-energy excitations of carriers can be determined in a contactless way in metallic and semiconducting SWNTs via ultra-broadband transient THz and mid-IR spectroscopy. (ii) We can utilize degenerate four-wave mixing to study the dephasing of coherent polarizations. Experiments are performed on resonance to the $E_{11}$ and $E_{22}$ absorption lines of semiconducting SWNTs in the near-infrared and visible regions. We investigate SWNTs individually dispersed in micelle or polymer surfactants which is essential to avoid extrinsic nanotube-nanotube interactions.

Accomplishments

We have successfully fabricated carbon nanotube samples suitable for $\mathrm{THz}$ and infrared work, by embedding SWNTs in PMMA, PVA, PAA and PVP polymers. Besides HiPco samples, we also employ CoMoCAT-grown specimens which show a narrow distribution dominated by $(6,5)$ and $(7,5)$ tube chiralities. Fluorescence characterization was also performed. Transparent films are important for our studies, and we are developing new techniques for spin coating layered samples to enable maximal spectral transmission in the mid-IR.

Using these polymer-based samples, we performed a number of experiments in the $\mathrm{THz}$ and mid-IR range. Optical-pump THz-probe experiments were employed as a contact-less probe of both the real and imaginary parts of the high-frequency conductivity. We observe transport properties at room temperature that underscore an excitonic nature of the photoexcited state, along with low-energy resonances. Tuning the excitation wavelength allowed us to test the resonance behavior of the photoexcitation process around the $E_{11}$ and $E_{22}$ transitions. We further constructed an optical pump mid-IR probe setup. First experiments have been carried out, where very strong photoinduced mid-infrared absorption has been observed for the first time in carbon nanotubes, including spectroscopic features associated with a bound excitonic state.

Furthermore, we performed the first three-pulse photon echo peak shift (3PEPS) experiments to elucidate delocalization behavior of excitons in semiconducting SWNTs. Via studies on $(6,5)$, $(7,5)$ and $(8,3)$ nanotubes, we extracted the decay time and analyzed the inhomogeneous distribution of transition frequencies. Systematic studies on various SWNT/polymer films and aqueous solutions using 3PEPS spectroscopy further enable us to access intrinsic exciton dynamics and to elucidate the influence of nuclear modes. 


\section{Carbon Based Materials for Renewable Energy \\ Alessandra Lanzara and Dung-Hai Lee}

\section{Project Description}

The focus of this project is to explore the possibility of using graphene as the host substrate for the hydrogen storage. We approach this problem both experimentally and theoretically. On the experimental side we carry out angle-resolved photoemission spectroscopy on graphite to study the novel Fermi point which is responsible for the unusual electronic properties of graphene. Theoretically we shall calculate the modification of graphene's screening property due to applied gate voltage or magnetic field.

\section{Accomplishments}

We have made the first direct observation of massless Dirac fermions with linear dispersion near the Brillouin zone (BZ) corner H in graphite, coexisting with quasiparticles with parabolic dispersion near another BZ corner K. In addition, we observed a large electron pocket which we attribute to defect-induced localized states. Thus, using graphite we have shown that massless Dirac fermions, quasi-particles with finite effective mass, and defect states all contribute to the low energy electronic dynamics. On the theory side we have examined the effect of the relativistic dispersion on the screening properties of graphene. We have shown that due to the vanishing of density of states, the Debye-Huckel screening length diverges. Consequently the classical picture of metallic screening is not applicable at any length scale. We have shown that when a unit charge is brought above a sheet of graphene, the image charge is about $2 / 3$ of the original. In contrast when the Fermi level moves away from the Dirac point, the full unit image charge is restored. Such a difference results in a delicate change of the attractive force between the charge and the substrate. We suggest to study this intricate modification of the screening property of graphene as a function of gate voltage by studying the change of interaction between the cantilever of a atomic force microscope and graphene. 


\section{SCIENCE AND TECHNOLOGY OF QUANTUM MATERIALS}

Senior Participants (LBNL \& UCB): R. Birgeneau (Physics), A. Lanzara (Phys), D-H. Lee

(Phys), J. W. Orenstein (Physics), R. Ramesh (MSE\&Physics), Y. Suzuki (MS\&E), A.

Vishwanath (Physics).

\section{Project Description:}

"Quantum materials,"systems in which the quantum-mechanical correlations of electrons play a dominant role, constitute a rich and diverse palette of physical phenomena. In such materials interacting charge, spin, orbital, and lattice degrees of freedom allow for a broader range of phase space, exemplified in the perovskite manganites, cuprates, ruthenates and cobaltates. At LBNL, we have brought together an interdisciplinary team of researchers, including theorists (Lee, Vishwanath, Moore) thin-film synthesis (Ramesh and Suzuki), and characterization (Browning, Birgeneau, Hellman, Lanzara, and Orenstein). Our LDRD project is probing novel quantum phases and their phase transitions in carefully prepared single crystals and comparing/contrasting these observations to those in epitaxial thin film heterostructures.

\section{Accomplishments:}

Orenstein and Ramesh collaborated on understanding spin dynamics in a model correlated system, $\mathrm{SrRuO} 3(\mathrm{SRO})$ and $\mathrm{SRO} / \mathrm{BiFeO} 3$ heterostructures to explore the interactions between a ferromagnet and a multiferroic. In epitaxial thin films, the optical measurements show the existence of a clear ferromagnetic resonance at a relatively high frequency of $\sim 250 \mathrm{GHz}$. Our studies on the SRO/BFO heterostructures suggest the possibility of exchange bias interactions, which are being verified by the THz measurements.

The collaboration between Ramesh, Suzuki and Orenstein focused on exploring new classes of correlated materials with the potential for multiferroic behavior, such as vanadiumbased perovskites. Two publications have been submitted on this work. In order to study electronic reconstruction at the interface of two dissimilar complex oxide materials, this year we have been focusing on the growth of $\mathrm{LaAlO} 3 / \mathrm{SrTiO} 3$ bilayers sandwiched in between metallic oxide electrodes of SrRuO3. These multilayers form a model system in which the lateral charge reconstruction across the $\mathrm{LaAlO} 3 / \mathrm{SrTiO} 3$ interface could be probed via tunneling measurements. Optical and electrical measurements are in progress and will continue under the DOE funded program.

The theoretical work of Moore and Vishwanath addressed electrical and thermal transport at a two-dimensional quantum phase transition into a superconducting phase. During this LDRD, the unusual properties of $\mathrm{MnSi}$, an itinerant ferromagnet which displays very unusual magnetic and transport properties was studied in collaboration with postdoctoral associate Dr. Benedikt Binz. A theory of a novel kind of magnetic structure - 'helical spin crystal' was developed in a series of papers, which agrees well with several experiments on this material. Dr. Tiago Ribeiro, a postdoctoral fellow working jointly with Profs. Dung Hai Lee and Vishwanath, obtained new results for the underdoped side of the cuprate high temperature superconductors using his 'doped carrier' formalism. In addition to specific results obtained in these studies, this research lead to the development of new theoretical techniques (eg. doped carrier formalism, a variant of slave-boson theory) which will be very useful in future studies of correlated materials under the Quantum Materials umbrella.

Our work on coupling of order parameters in perovskites such as $\mathrm{BiFeO} 3$ blossomed very well and is poised to make a significant worldwide impact. Several publications (see list) are already in press and more papers will come out over the next few months.

This LDRD has been successfully transitioned into a proposal to DOE that has already been funded by DOE starting October 2006. 


\section{A Polarizer System for Microfluidics}

\section{Alex Pines}

\section{Project Description}

The project goal is to use para-hydrogen to enhance the polarization of nuclear spins and to couple para-hydrogen techniques to microfluidic devices in order to be able to use sensitive low field detection methodologies such as laser-based atomic magnetometry at the earth's magnetic field for enhanced analyses and imaging at low cost. The creation of para-hydrogen induced polarization can be achieved through the catalyzed hydrogenation or hydroformylation of a substrate molecule. The reaction can take place inside or outside the magnetic field and should result in enhanced NMR peaks and allow for the storage of polarization over extended periods of time. The para-hydrogen can be coupled with micro-flow systems for studying the kinetics of chemical reactions, coupling topologies in the earth's field, and profiles of various physical processes such as flow. These approaches directly interface with "lab-on-a-chip" devices, multiplexed micro-total analysis systems ( $\mu$-TAS) and systems for on-line monitoring of biological and pharmaceutical assays.

One advantage of the para-hydrogen induced polarization is that the polarization achieved can approach 100 percent and this effect is independent of the field strength. Thus, enhancements by 5 to 10 orders of magnitude are possible in magnetic fields ranging from $20 \mathrm{~T}$ to the earth's field respectively. Para-hydrogen can dramatically increase the sensitivity of low-field detection methodologies in cases when the concentration and volumes of the analyte are too low for observing thermal polarization. These alternative detection schemes are especially promising for mobilized, miniaturized, low-cost and cryogen-free spectroscopic and imaging sensors.

\section{Accomplishments}

Construction of the polarization setup for the synthesis of nearly pure para-hydrogen, by cooling down hydrogen gas to liquid helium temperatures has been completed and $50 \%$ parahydrogen was achieved by cooling in a liquid nitrogen environment. Work was done on the optics setup for the delivery of laser light into the magnet for photolyzed reactions, initiating and terminating the addition of the pure para-hydrogen molecules to the substrate. Pulse sequences based on optimal control theory for the efficient transfer and shuffling of polarization from the polarized hydrogen spins to other nuclei in the same molecule were developed. In collaboration with Robert Bergmann's group, (Chemical Sciences, LBL), we have synthesized the molecules that will be used for the photo-triggered reaction.

Important progress was made with heterogeneous hydrogenation using para-hydrogen, in collaboration with I. Koptyug from the International Tomography Center, Novosibirsk, Russia. Three different supported rhodium catalysts were used to demonstrate, for the first time, the creation and observation of para-hydrogen induced polarization in heterogeneous hydrogenation reactions. These unprecedented results show that para-hydrogenation with heterogeneous catalysts can be developed into a practical tool for producing catalyst-free fluids with highly polarized nuclear spins for a broad range of hyperpolarized NMR and MRI applications. As a first step, we have built a flow-through catalytic reactor polarizing fluids as they pass through and subsequently, demonstrated imaging of the hyperpolarized product in a phantom. Results from the heterogeneous catalysis also open up the possibility of immobilizing the catalyst on microchannels in a microfluidic device. 


\title{
Aging, Disease and the Mechanical Response of Biological Tissues, Specifically Human Bone
}

\author{
Principal Investigators: R. O. Ritchie, J. W. Ager, A. P. Tomsia, and C. A. Larabell
}

\section{Project Description}

A major new effort was initiated to determine how diseases, therapeutic treatments, and aging affect the mechanical properties of biological mineralized tissue such as bone and dentin. Our approach is fundamentally different in that it is designed to evaluate so-called bone quality, as opposed to the methods used at present for most clinical and research studies which concentrate on bone quantity. Our approach is motivated by evidence that changes in bone quantity that occur as a result of treatments for osteoporosis or are present between different population groups explain only a fraction of the differences in fracture risk. In this context, our approach is aimed at bridging the gap between the existing clinical understanding of bone fracture and the underlying micromechanical mechanisms of bone failure.

We employ quantitative methods that span the relevant hierarchical length scales associated with fracture in bone and dentin. At the heart of our approach are detailed fracture mechanics measurements aimed at delineating the mechanism by which mineralized tissues resist (or, fail to resist) crack propagation. These are performed in situ in both scanning electron and optical microscopes. Measurements at the nano and molecular scales (pico-force AFM, nanoindentation, $\mathrm{x}$-ray scattering, and vibrational spectroscopies) and microscale (computed hard and soft x-ray tomography) are used to determine the underlying structural determinants of the fracture behavior.

\section{Project Accomplishments}

In our FY06 effort, we investigated the effects of aging on human bone and teeth and participated in two studies with UC Davis (Nancy Lane) in which the effects of systemic steroids were investigated in a mouse model and the effects of therapeutic treatments for osteoporosis were evaluated in an ovariectomized rat model. Our in situ fracture mechanics studies are revealing the dominant toughening mechanisms in mineralized tissues. In teeth, these are bridging behind the crack tip and microcracking of the tubule lumens ahead of the crack tip. In older dentin, the lumens are filled with mineral precipitates and this latter mechanism does not occur, which leads to reduced fracture toughness. In bone, crack bridging and crack deflection at "cement lines," which are defined by the ongoing osteonic remodeling process, contribute to crack resistance. As human bone ages, we have found that it is less resistant to crack propagation because of a reduction in the efficacy of crack bridging, which we believe is associated with a higher density of (secondary) osteons from excessive remodeling. By using measurements of the changes in the mineral phase (small angle $\mathrm{x}$-ray scattering and AFM-based modulus mapping) and organic phase (collagen-specific ultraviolet resonance Raman spectroscopy) in both human bone and in controlled animal studies, a consistent picture of how changes in the bone-matrix nano-/micro-structure control the fracture mechanisms is emerging.

Our studies on disease and clinical treatment in their effect on the structure and toughness of bone are still in their infancy; however, it is through such a multi-dimensional mechanistic approach, which combines biology, materials science, and fracture mechanics, that a clearer understanding of what toughens and embrittles bone can be achieved. We believe that such information can be used as basis for the design of improved drug therapies to reduce the risk of bone fracture in the elderly and in other at-risk populations. 
Electron Flow Generated by Gas Phase Exothermic Catalytic Reactions Using MetalSemiconductor Nanodiodes Principal Investigator: Gabor A. Somorjai

\section{Project Description}

When heat is deposited as a result of exothermic surface reactions, the electrons heat up much faster (femtoseconds) than the lattice (picoseconds) because of their much lower heat capacity. The electron flow is detected as a chemicurrent if the excess electron kinetic energy generated by the exothermic reaction is larger than the effective Schottky barrier formed at the metal-semiconductor interface. The purpose of this project is to utilize hot charge carriers generated by an exothermic catalytic reaction to detect or even control the chemical reaction and to understand the role of electronic transport of hot electrons through the metal-oxide interface in exothermic metal catalyzed reactions.

We will explore the mechanism of continuous hot electron current generation by 1) systematically tailoring the semiconductor and metal catalyst materials; 2 ) altering the catalytic nanodiode structure to include thin films, nanowires, and nanoparticles; 3 ) changing the exothermic catalytic reaction to study the oxidation of methanol, hydrogen and carbon monoxide, the hydrogenation of hydrocarbons, and combustion. We shall explore how the hot electron flow influences the catalytic chemistry of the metal catalysts by correlating current flow with catalyst selectivity, activity and stability. Transport mechanisms will be investigated as a function of doping density and Schottky barrier height.

\section{Accomplishments}

We fabricated two types of catalytic Schottky nanodiodes, $\mathrm{Pt} / \mathrm{TiO}_{2}$ and $\mathrm{Pt} / \mathrm{GaN}$, with various microelectronic techniques. The flow of hot electrons during $\mathrm{CO}$ oxidation (at pressures of 100 Torr of $\mathrm{O}_{2}$ and 40 Torr of $\mathrm{CO}$ at $373 \sim 523 \mathrm{~K}$ ) and hydrogen oxidation (at pressures of 100 Torr of $\mathrm{O}_{2}$ and 1-4 Torr of $\mathrm{H}_{2}$ at $273 \sim 373 \mathrm{~K}$ ) were measured. The results reveal that exothermic catalytic chemical reaction energy is directly converted into hot electron flux in the catalytic nanodiode. The chemicurrent is well correlated with the turnover rates of $\mathrm{CO}$ oxidation and hydrogen oxidation, which were separately measured with gas chromatography.

We have focused on optimizing catalytic nanodiode characteristics for maximum hot electron current flow. We have characterized the electrical, chemical, mechanical, and topographical characteristics of catalytic nanodiodes by using current-voltage measurement, Auger spectroscopy, X-ray photoemission spectroscopy, and atomic force microscopy. We have studied the influence of physical and chemical properties on the chemicurrent yield (number of hot electrons per product molecule). The $\mathrm{Pt} / \mathrm{GaN}$ diode was found to have a higher chemicurrent yield than the $\mathrm{Pt} / \mathrm{TiO}_{\mathrm{x}}$ diode. This is attributed to an atomically smooth Pt-GaN interface, as measured with AFM, leading to fewer scattering centers and a higher transmission probability of hot electrons.

We are in the process of exploring both $\mathrm{n}$ - and p-type GaN semiconducting materials in order to generate and control hot electrons and holes. 
Computational and theoretical studies of bulk and nano solid systems Principal Investigators: Ivo Souza and Steven G. Louie

\section{Project Description}

The purpose of this project is to carry out theoretical and computational investigations of magneto-optical and magneto-transport properties in ferromagnetic metals. Examples of such properties are magnetic circular dichroism (the difference in absorption between left- and rightcircularly-polarized light) and the anomalous Hall effect (the voltage that develops in the direction perpendicular to an electrical current and the sample magnetization). These properties result from subtle modifications of the band structure and electronic wave functions caused by the exchange splitting and the spin-orbit interaction. An accurate $a b$-initio description requires sampling the Brillouin zone (BZ) at millions of k-points to converge the k-space integrals. One goal of the project is to develop efficient techniques for carrying out the BZ integrations with low cost and high accuracy, which we termed "Wannier interpolation." The second goal is to use these novel and powerful tools to gain a better understanding of the mechanisms which give rise to these effects in real materials. The tools developed in the course of this problem are readily applicable to other problems in the physics of metals which require denze BZ sampling. An important example is the electron-phonon interaction needed to study BCS superconductivity.

Accomplishments

The Wannier-interpolation technique was successfully developed and applied to compute the anomalous Hall conductivity of ferromagnetic iron. A calculation which before required weeks of computer time can now be done in a few hours on a desktop computer, with higher accuracy. This will allow studying more complex materials than was previously possible. The relative contributions from like-spin and opposite-spin portions of the Fermi surface to the net effect was elucidated. The Wannierinterpolation technique has also been extended to compute the electron-phonon interaction, and applied to study the superconductivity in boron-doped diamond, shedding new light on the underlying mechanism. We are in the process of extending the method to study finite-frequency magneto-optical properties and the low-field Hall effect of paramagnets and diamagnets. The connections between these and other spin-orbit-induced phenomena, such as orbital magnetization, are being studied theoretically via subtle sum rules. This will allow to test our predictions in the laboratory using synchrotron radiation sources, such as the Advanced Light Source at the Lawrence Berkeley National Laboratory.

The source codes developed in the course of the project have been made available to the electronic-structure community at large by releasing them under the Gnu Public Licence (GPL) at the web site http://www.wannier.org. 
Experimental Signatures of Fractionalized Phases and Phase Transitions in Strongly Correlated Electronic Systems

P.I. ASHVIN VISHWANATH

Project Description

The main focus of this project was to understand some of the conditions under which strongly interacting many-particle quantum systems are described in terms of fractionalized excitations (excitations that carry fractional quantum numbers) and the resulting experimental signatures. Recently, there has been an improved understanding of the universal features of such states (for example they are necessarily accompanied by an emergent gauge theory in its deconfined phase), both as stable phases and as quantum critical points. However the microscopic conditions under which these states may arise, and also their characteristic experimental fingerprints are less well understood and this was sought to be remedied by the present study. Ultimately, it is hoped that with a better understanding of these issues will help answer the question of which strongly correlated materials controlled by this interesting new physics.

\section{Accomplishments:}

1. In collaboration with my student, Fa Wang, we studied spin liquid states that might be stabilized in quantum antiferromagnets on frustrated lattice geometries such as triangular and Kagome. While previous works have taken mean field energetics as the sole determinant of which states to consider, here we brought to bear a powerful symmetry based approach (Projective Symmetry Group) that allows one to access all relevant states. This was applied to both the triangular and kagome lattices, and new candidate bosonic spin liquid states were obtained which are especially resistant to spin order. Interactions that could potentially stabilize these phases, such as further neighbor exchange and ring exchange were also presented. This was published in Physical Review B.

2. In collaboration with my postdoctoral associate Daniel Podolsky, and Sri Raghu (a student at Princeton University) we investigated the Nernst effect and magnetization in a thermally fluctuating superconductor, in the regime where superconductivity is destroyed by phase fluctuations. Such a situation is of relevance to describing the underdoped copper oxide superconductors. We find good agreement with existing data on these materials, and made a number of nontrivial predictions, such as the close quantitative relation between diamagnetism and Nernst effect in these systems. This work has been submitted for review to Physical Review Letters. An outgrowth of this work was a technique for directly simulating dynamics of fluctuating superconductors using vortex variables which allows one to study nontrivial dynamics associated with fractionalized states. This is currently under study.

3. In independent work, my student Fa Wang studied the partition function of classical hard core dimmers on the Kagome lattice. This is related to the properties of the Resonating Valence Bond wave function of a spin liquid on the triangular lattice. This work has been submitted to Physical Review Letters. 


\title{
Nuclear Science Division
}

\section{LB04017}

\section{Research and Development for Double Beta Decay Experiments}

\author{
Principal Investigator: Kevin Lesko
}

\section{Project Description}

With the success of the Sudbury Neutrino Observatory in convincingly demonstrating neutrino transformations, resolving the Solar Neutrino Problem, and also proving that neutrinos have mass, and with the KamLAND experiments refinement of the oscillation parameters we are still faced with several fundamental questions about neutrinos: the absolute mass of neutrinos (the oscillation experiments have measured the differences in masses between the species), the ordering of the neutrino masses between the three known neutrino flavors (the mass hierarchy), and the exciting prospect of determining if the neutrino is its own antiparticle. This characteristic of massive particle being its own antiparticle would make the neutrino unique. Neutrinoless double beta decay experiments can deliver evidence leading to the determination of the absolute neutrino mass, it can determine the hierarchy of the neutrino masses, and, most importantly, provides the only technique to determine if the neutrino is Dirac or Majorana in nature (if it is its own antiparticle). The importance of double beta decay has been recently highlighted in the American Physical Society Neutrino report (www.aps.org) stressing that next generation DBD experiments are the highest priority for the US neutrino community. Recently the Department of Energy has evaluated neutrinoless double beta decay experiments with their Neutrino Scientific Assessment Group and several technical reviews.

The purpose of this project is to develop key technologies, data analysis tools, and comprehensive simulation packages essential for the success of next generation neutrinoless double beta decay (0nDBD) experiments. There are several leading candidate for the next generation of OnDBD experiments, one using Ge-diodes to detect the energy as ionization, and another using $\mathrm{TeO}$ cryogenic crystals to detect the energy as heat. Because of the exceedingly long lifetimes for $0 \mathrm{nDBD}$, radioactive backgrounds are the major obstacle for these experiments. The Ge experiment draws on nearly four decades of experience operating Ge-diodes. The Te cryogenic technique has made great progress in the past decade in developing and operating small scale arrays of TeO crystals. However, controlling, understanding and identifying backgrounds in both techniques remains the greatest challenge for this field. A variety of topics have been undertaken to achieve this goal of reducing the sensitivity of the experiments to backgrounds through the physical reduction of the radioactive backgrounds, by investigating novel detector designs and operating procedures to identify and separate backgrounds signals, and by developing comprehensive simulation packages. In addition, we are developing data analysis strategies and tools to reduce and interpret data from the experiments.

\section{Progress and Accomplishments}

For the Majorana collaboration we developed and maintain the comprehensive Monte Carlo simulation package, MaGe, which is the main tool for Majorana Monte Carlo Analysis and detector evaluations. Our efforts have predominantly focused on using modern simulation tools from the high energy and nuclear physics communities to assemble a modern experimental simulation package that can be shared with the entire $(\sim 100$ member) collaboration. This simulation package we handle the detector geometries, media properties, shielding characteristics, and all interesting physics interactions for the Majorana experiment. We continue to work with the GRETINA project in the NSD, to develop novel detector segmentation schemes and data analysis capabilities to reduce the sensitivity of the experiment to internal and external backgrounds, primarily gamma-rays. To better understand the irreducible background generation consequences of producing Ge detectors above ground we have initiated studies into the feasibility of building Ge crystals and detectors in underground, shielded environments. $\mathrm{MaGe}$ was the focus of the segmentation evaluation completed by the collaboration with significant Berkeley Lab input.

For the Cuoricino project we have split our efforts between 1) data analysis techniques and building from scratch an analysis program to confirm the European efforts, 2) research in producing thermistors essential to the success of the project, and 3) investigating TeO crystal production and contamination. The data analysis chain was completed 2005, in time for the summer conference cycle. Thermistor production saw significant progress in understanding and measuring the neutron dose needed to create the thermistors. We have identified a crystal producer in the Bay Area and are discussing a variety of novel crystal bonding techniques that would remove a significant uncertainty in the mass-production of the cryogenic crystals. We continued to develop low background material assays. 
Establishing New Capabilities in Nuclear Astrophysics and Radiation Biology using Neutrons at the 88-Inch Cyclotron

Principal Investigator: Margaret A McMahan

Project Description

This is the second and final year of a project to explore the extension of newly developed neutron capabilities at the 88-Inch Cyclotron to other applications.

The Phase 1 neutron facility, which was funded through another grant, was constructed with space for two endstations. This project funded some key experiments as initial applications of this capability:

1) In the Cyclotron vault, beams of quasi-monoenergetic neutrons with energies from 10-32 $\mathrm{MeV}$ are produced by Coulombic dissociation of deuterons in thin targets. The purpose of the new facility is to measure the cross sections for neutrons interacting with radioactive targets. One part of the LDRD supported the exploration of options for producing lower energy neutrons for nuclear astrophysics measurements.

2) In Cave 2, a broad energy beam will be available for radiation effects testing of biological systems and electronics as well as other applications. This LDRD funded research and development necessary to implement this capability, which we are calling the BASE Neutron Facility, BASE-NF.

Accomplishments

This year, work progressed towards BASE-NF, using an existing beamline in Cave $3 \mathrm{~b}$ to characterize neutrons from $20 \mathrm{MeV}$ deuterons impinging on a thick beryllium target. A measure of the neutron time of flight was used to determine the energy spectra of neutrons at $0^{\circ}$ and $40^{\circ}$ and compare them to literature values. The absolute neutron flux/steradian was determined using activation foils. A successful benchmark experiment was performed in November 2006 using BASE-NF for researchers in Physics Division.

The initial design of the production endstation in Cave 2 was completed, and a series of runs were performed to determine the need to upgrade the existing radiation shielding when running BASE-NF experiments at high intensities. Work is continuing under follow-on funding.

A set of detectors, electronics and data acquisition has been assembled using existing equipment. For BASE-NF, the long term goals include the use of position-sensitive detectors to image the neutron beam across several inches. During the summer, a prototype fission chamber was designed and built by undergraduate interns as part of this effort. The prototype did not perform at the level needed but gave vital information that has been used to set the requirements needed for such a detector; commercial avenues are now being explored.

This LDRD also played an important role in establishing a collaboration of scientists from several divisions (NSD, LSD, EH\&S) and outside institutions (UCB and LLNL) in order to develop detectors, characterize the neutron beams and perform exploratory experiments with applications in Homeland Security (for Engineering Division), future detector development for the ILC (for Physics Division), and NIF diagnostics (for LLNL). We are well positioned to pursue current and future opportunities for specific experiments, for example, from NDNO, DOE Office of Science or Nuclear Energy (AFCI Initiatives) and LLNL through the NIF program. 
Improved Spectroscopy of Weakly Bound States in Nuclei

Larry Phair

\section{Project Description:}

Future nuclear structure measurements will take place at the drip lines of very neutron-rich and proton-rich nuclei. These nuclei will be identified by detecting the last particle stable isotope and the spin and parity of its ground state and low excited states, if any. By studying states at high excitation energy now (i.e., the unpopulated drip line states), we can explore in a novel way the single particle, pairing, and vibrational properties of states more commonly studied near the Fermi surface in ordinary nuclei.

Our goal is to demonstrate the feasibility of studying the future drip line single particle and collective states, by populating these states which lie about 6-7 MeV above the Fermi surface in ordinary nuclei. If this technique works, a program could be implemented to study drip line states via direct reactions and gamma-ray measurements using the high efficiency GRETINA array.

\section{Accomplishments:}

Direct reactions have been measured using the light-ion beams from the 88-Inch Cyclotron. The reaction ${ }^{208} \mathrm{~Pb}(d, p \gamma){ }^{209} \mathrm{~Pb}$ was performed to populate single particle states in ${ }^{209} \mathrm{~Pb}$ both near the surface and at higher excitation energies as well. States near the surface were identified with both charged particles and coincident gamma rays. We used the gamma rays detected with high resolution in the Ge crystals to select the neutron states at high excitation. The detected charged particles were used to establish the excitation energy of the nucleus. However, the limited solid angle of our set-up prevented us from measuring angular distributions of the charged particles with sufficient range so as to characterize the states in terms of spin and parity. During the last year we doubled the solid angle of charge particle detection and performed a separate experiment, ${ }^{173} \mathrm{Yb}(d, p \gamma){ }^{174} \mathrm{Yb}$, with improved angular coverage. The larger angular coverage should allow for a spin characterization of observed states. The analysis of this new experiment is in progress.

In the second experiment called for in the LDRD, a beam of ${ }^{17} \mathrm{O}$ at $380 \mathrm{MeV}$ was used to excite a ${ }^{208} \mathrm{~Pb}$ target. The heavy ions were used to inelastically excite collective states in ${ }^{208} \mathrm{~Pb}$. The scattered ${ }^{17} \mathrm{O}$ was detected in the silicon detectors and the gamma rays de-exciting high-lying sates in ${ }^{208} \mathrm{~Pb}$ were measured with Ge detectors. Previous experiments used low-resolution gamma-ray detectors $\left(\mathrm{NaI}\right.$ and $\left.\mathrm{BaF}_{2}\right)$, and while resonances, in particular the pygmy dipole resonance (PDR), were excited, no detailed structure was seen. Using the high-resolution Ge detectors, we have observed detailed structure of states in the PDR region. Unfortunately, the silicon detectors only lasted one day because the high particle flux led to high leakage currents which ruined the detector resolution for identifying ${ }^{17} \mathrm{O}$. To remedy this, we have designed and constructed a refrigeration unit that will allow us to cool the silicon detectors and lower the leakage currents. Upon successful testing of the cooling technique, a new run will be scheduled in the spring. With larger statistics, we will attempt to resolve individual states with increased statistics up to and beyond the pygmy region and establish the decay pathways of these states. 
Conceptual Study for a Novel Nuclear Astrophysics Accelerator Facility Principal Investigators: Paul Vetter, Daniela Leitner, Mattheus Leitner

Project Description

We seek to design a new high-current, low-energy accelerator facility which can be used to precisely measure the nuclear fusion reactions which power main-sequence stars similar to our sun. To accurately predict nucleosynthesis rates, stellar and galactic evolutions, and isotopic abundances, the reaction cross sections for stellar hydrogen and helium burning phases need to be more accurately known. These cross-sections must be measured, in many cases, at energies so low that laboratory reaction rates have hitherto been vanishingly small. The yield at low energies can be increased by using very high beam currents - more than a factor of 100 higher than at existing or planned facilities. Substantial R\&D is necessary to design such high-intensity beams. A dense gas-jet target offers well-controlled systematic uncertainties, and R\&D is necessary to integrate the highest achievable jet target densities with high beam currents and detection instrumentation. Some of the reactions we seek to measure are ${ }^{3} \mathrm{He}\left({ }^{4} \mathrm{He}, \gamma\right)^{7} \mathrm{Be}$, $\left.\mathrm{t}\left({ }^{4} \mathrm{He}, \gamma\right)\right)^{7} \mathrm{Li}, \mathrm{d}(\mathrm{p}, \gamma){ }^{3} \mathrm{He}, \mathrm{p}(\mathrm{n}, \gamma) \mathrm{d},{ }^{14} \mathrm{~N}(\mathrm{p}, \gamma){ }^{15} \mathrm{O},{ }^{7} \mathrm{Be}(\mathrm{p}, \gamma) 8 \mathrm{~B},{ }^{7} \mathrm{Li}(\mathrm{d}, \mathrm{p}){ }^{8} \mathrm{Li}$, and ${ }^{17} \mathrm{O}(\mathrm{p}, \alpha){ }^{14} \mathrm{~N}$

Accomplishments

During FY06, we designed the ion optics for a $300 \mathrm{kV}$ accelerator consisting of a high voltage platform, several magnetic solenoids and a $60^{\circ}$ bend magnet. Extensive simulations using the first order matrix code TRACE3D, the 3D Particle-In-Cell code WARP and the 2D ion beam trajectory code IGUN were performed to optimize the transport, mass analyzing magnet, and the final focusing spot of a $100 \mathrm{~mA}^{3} \mathrm{He}$ beam at $50 \mathrm{keV}$. For these high intensity beams, space charge is the dominant issue in beam transport. To focus the beam to $5 \mathrm{~mm}$ at the target area, it will be necessary to partially or fully neutralize the high intensity ion beam in the beam transport line. In our simulations we have assumed a $70 \%$ neutralized beam ( $30 \mathrm{~mA}$ effective charge). Ion beam neutralization of $99 \%$ has been reported in the literature for beam line pressures of $10^{-4}$ mbar. However, neutralization in an analyzing bend has not been experimentally verified so far.

We have developed a detailed list of requirements for the astrophysics accelerator facility. High beam currents $(100 \mathrm{~mA}$ to $1 \mathrm{~A})$ with tight focus $(<1 \mathrm{~cm})$ and a narrow beam energy distribution ( $\sim .1 \%$ of the beam energy) are necessary. A cooled $(40 \mathrm{~K})$ gas jet with density $3 \times 10^{17}$ atoms $/ \mathrm{cm}^{2}$ would generate a $1 \%$ statistical uncertainty for the ${ }^{3} \mathrm{He}\left({ }^{4} \mathrm{He}, \gamma\right){ }^{7} \mathrm{Be}$ reaction cross section in 1 day of operation at a center of mass energy of $80 \mathrm{keV}$. We have narrowed down the accelerator options, identified the need for a supersonic gas jet target, and investigated using segmented germanium detectors for the first set of experiments - identifying a regime of relatively low-energy final state gamma rays in which backgrounds from natural radioactivity are not prohibitive for low-rate experiments.

In addition, we have strengthened the science case and support for such a facility. We have gained significant support (including letters of support for DUSEL R\&D funding) from the nuclear astrophysics community, NSD, and ED. 


\section{Physical Biosciences Division}

\section{LB05044}

The Statistical Dynamics of Protein Evolution

Principle Investigators(s): Gavin E. Crooks

\section{Project Description}

The goal of this project is to create the next-generation algorithms for the automatic reconstruction of protein evolution from protein sequences. This requires a mathematically elegant, computational efficient and biological realistic model of protein sequence evolution over long time scales; an efficient Monte-Carlo algorithm for sampling from the posterior distribution of protein histories; and reliable benchmarks to validate the authenticity of the reconstructed histories. Three particular deficiently with existing algorithms will be addressed; the simulations inferences of sequence alignment and protein evolution using a consistent algorithm; the higher level evolution of proteins through domain reorganization; and the computational challenges that must be faced when considering the vast and rapidly growing size of the protein sequence database.

\section{Accomplishments}

We have been investigating statistical relationship between the amino acid sequence of a protein and it's structure, and how these relations are preserved (or not) during evolution. Surprisingly, (but in accord with recent published research) amino acids that are neighbors in the structure, but distant on the chain are almost uncorrelated. This implies that proteins are held together by subtle diffuse interactions between many residues, and not by strong direct pairwise interactions, as is often assumed. We have significantly improved the previous statistical analysis in the area.

We have also significantly updated WebLogo (http://bespoke.lbl.gov/weblogo), the leading application for generating sequence logos, graphical representations of the patterns in an amino acid or nucleic acid multiple sequence alignment. The most important improvement is a new method for reliable estimating entropy from finite data sets, and for estimating reliable error bounds. This method was initially developed for the investigation of sequence correlation in evolution.

Finally, research in protein evolution has led to new insights in nonequilibrium statistical physics, as detailed in the paper "Nonequilibrium maximum-entropy hyperensembles: Beyond Boltzmann-Gibbs statistics" 


\author{
High-throughput Production of Proteins and Protein Complexes \\ Principal Investigator: Thomas Earnest
}

Project Description

To attempt a genomic-scale production of multi-protein complexes, methods and technologies need to be developed that allow for the determination of which sets of constructs will produce large amounts of these samples in the soluble fraction for purification, or can be refolded. Searching for the correct constructs or sets of constructs would benefit greatly from the development of parallelized screening methodologies and their implementation into an automated and intelligently controlled instrumentation environment. One reason that protein production is challenging for some targets, particularly eukaryotic targets, is the requirement for another protein or set of proteins to bind to the protein to maintain solubility, which are complexed physiologically with the protein in its normal cellular environment. The identification of ensembles of proteins that form complexes are guided by existing information available from the DNA microarray analysis, two-hybrid studies, and tandem affinity purification (TAP) coupled with mass spectroscopy to investigate and identify biomolecular interactions. Once interacting partners are determined, co-expression of sets of constructs of these proteins along with expression and mixing to form complexes are attempted. If these experiments do not yield sufficient amounts (milligrams) of soluble complexes, the proteins are engineered utilizing gene shuffling and/or error-prone PCR to evolve soluble protein complexes. Initial target samples include multi-protein complexes from prokaryotic (Caulobacter crescentus) and eukaryotic (yeast, Drosophila melanogaster) sources. Caulobacter crescentus, a microbial system that has promise as a bioremediation agent, is our primary focus in collaboration with Harley McAdams and Lucy Shapiro of Stanford. The yeast Saccharomyces cerevisiae has been well characterized in particular in regard to transcriptional regulatory pathways. Both organisms have sequenced genomes and are well studied by a variety of genetic and biochemical methods, thus providing a basis for biological and cellular understanding of the roles these complexes mediate. We also plan to utilize the structures that we can obtain from these complexes to attempt to localize them in the cell in space and time. In this regard, we collaborate with Ken Downing on cryo-EM tomography, as well as develop Caulobacter as a model system for diffractive imaging experiments with John Miao of UCLA, and Qun Shen and Ian McNulty of the APS

\title{
Accomplishments
}

Working with the group of our collaborator, Gary Andersen of the LBNL Earth Sciences Division, we have been able to establish TAP tagging in Caulobacter, initially using the protein rcdA as a test case. TAP purification has indicated that the complex identified by coimmunoprecipitation is also present after this process. This serves as a valuable check on the success of the method, as this is the first time this approach has been established in this organism. We have also been able to integrate sequences into the genome that feature the Gateway-compatible regions, allowing for future use for a wide range of targets for a genomewide screen for protein-protein interactions. This large scale search for protein-protein interactions is underway.

Coherent $\mathrm{x}$-ray diffractive imaging studies have also produced initial data sets for reconstruction of Caulobacter and Saccharomyces. These images, while not optimised and from air-dried samples, yield high-resolution data that we plan to extend to three-dimensions using frozen-hydrated samples. 


\section{Synthetic Cytoskeleton: Protrusive Structures for Reconstituted Cell Motility Principal Investigator: Daniel A. Fletcher}

\section{Project Description}

The purpose of this project is to develop an experimental system for studying how the actin cytoskeleton contributes to crawling motility. Crawling motility is a fundamental cellular process essential for wound healing, immune response, and embryonic development. Synthetic crawling motility could have important applications for directed drug delivery in the body, pathogen detection, and environmental sensing, but a fundamental understanding of the cytoskeletal dynamics required for directed movement is lacking. This project aims to functionally reconstitute the first step of crawling motility - protrusion - using only purified cytoskeletal proteins in lipid vesicles.

Protrusive cytoskeletal structures will be reconstituted from a simplified system of purified proteins consisting of actin and associated nucleating, branching, and cross-linking proteins. In the first year, we characterized protrusion force and velocity of the simplified system in vitro using an atomic force microscopy (AFM) technique that we recently developed. In the second year, we investigated spatial organization and growth of protrusive structures in lipid vesicles by initiating actin polymerization at the membrane. We studied force distribution and growth rate of the synthetic cytoskeleton and its relationship to the concentration and spatial organization of molecular components. This work will lay a foundation for future efforts to rebuild the entire process of crawling motility and put synthetic directed movements to use for health and technology applications.

\section{Accomplishments}

Our major accomplishments in the second year of the project have been (1) development of an in vitro system to study the organization of actin networks on synthetic lipid vesicles and (2) observation of altered phase separation behavior in the actin-associated lipid membrane. Spatial organization of the plasma membrane and associated membrane proteins underlies many important cellular processes, including cell-cell communication, polarization, and motility. We hypothesized that membrane components linked through the cell's actin cytoskeleton could influence global membrane organization, providing a mechanism through which changes in cytoskeletal organization could directly alter membrane organization. This ability to control membrane organization will be critical for reconstituting crawling motility in lipid vesicles. Ternary lipid systems that undergo phase separation into coexisting liquid ordered and liquid disordered domains have served as model systems for investigating membrane organization in recent years. Phase separation behavior can be quantified with miscibility transition temperature, the temperature above which separated phases are homogenized into a single phase.

We found that formation of a localized actin network on model lipid membranes both induces de novo membrane domain formation and stabilizes existing membrane domains by shifting the miscibility transition temperature of the membrane. Furthermore, the presence of an actin network associated with the membrane was found to spatially bias location of membrane domain formation after homogenization. These results suggest that actin polymerization can act as a "switch" for spatial and temporal organization of membrane components. 


\section{Visible Light-Driven Water Oxidation in Mesoporous Solids \\ Principal Investigators: Heinz Frei and Vittal Yachandra}

Project Description

Direct visible light-driven splitting of water to hydrogen and oxygen, or reduction of $\mathrm{CO}_{2}$ by $\mathrm{H}_{2} \mathrm{O}$ to a C-based liquid fuel like methanol requires two or more solar photons driving the reduction and oxidation half-reactions. With current artificial approaches, the photosynthesis ceases rapidly unless sacrificial reagents are used to avoid back reaction and materials degradation, which renders the processes unsustainable. Moreover, existing synthetic water oxidation catalysts can only be driven electrochemically or by strong chemical oxidants rather than by visible light, a prerequisite for coupling of the oxygen evolving with the fuel forming reaction. Therefore, the main obstacles toward efficient visible light-induced water splitting or $\mathrm{CO}_{2}$ reduction are (i) the coupling of efficient water oxidation catalysts to photon-driven electron transfer chromophores on a heterogeneous support; (ii) the coupling of water oxidation and proton or $\mathrm{CO}_{2}$ reduction in a 3-D assembly that affords overall fuel production without the need for sacrificial reagents. The purpose of the proposed work is to address these obstacles.

We propose to address the challenge by exploiting mesoporous inorganic scaffolds for the coupling of biomimetic or inorganic oxide water oxidation catalysts with visible light chromophores embedded in the pore surface of the host structure. Taking guidance from nature's $\mathrm{Mn}_{4} \mathrm{Ca}$ water oxidation cluster in photosystem II, emphasis will be on oxo-bridged multinuclear Mn complexes tethered to the pore surface by suitable organic moieties, and organic-free transition metal oxide clusters. Mesoporous inorganic oxide scaffolds will be explored for coupling of the water oxidation units with binuclear $\mathrm{CO}_{2}$ activating photocatalysts for overall $\mathrm{CO}_{2}$ reduction by $\mathrm{H}_{2} \mathrm{O}$ under visible light.

\section{Accomplishments}

A photocatalytic unit for visible light water oxidation has been assembled in the nanometersized pores of a silica scaffold (MCM-41). The unit consists of an iridium oxide nanocluster covalently linked to a single $\mathrm{Cr}$ center embedded in the silica pore surface. The ligand-to-metal charge-transfer absorption of the $\mathrm{Cr}$ center, which absorbs in the blue and green spectral region, acts as a visible light electron transfer pump pulling electrons one at the time from the Ir oxide nanocluster. Evolution of oxygen gas from liquid water was monitored by mass spectrometry. This is the first observation of photo-driven water oxidation by a multi-electron transfer catalyst coupled to a molecular charge-transfer chromophore.

The unit was assembled on the silica nanopore surface by incorporation of tetrahedral $\mathrm{Cr}^{\mathrm{VI}}$ centers during hydrothermal synthesis of the scaffold. Exploiting the selective reactivity of $\mathrm{Cr}$ centers towards an organometallic $\mathrm{Ir}^{\mathrm{III}}$ precursor loaded from solution, Cr-O-Ir linkages were formed and directly observed by FT-Raman spectroscopy. Mild heating under oxygen formed Ir oxide nanoclusters whose presence in the nanopores was verified by high resolution electron microscopy (Z-contrast HR-TEM and NCEM) combined with X-ray spot analysis. The use of surface-anchored metal centers as charge-transfer chromophores for driving water oxidation opens up a method for coupling a visible light water oxidation unit to a $\mathrm{CO}_{2}$ photoreduction unit.

In parallel, we have synthesized and loaded several organometallic Mn dimer complexes capable of water oxidation onto Cr-doped silica nanopores. Through observation of the characteristic 16-line EPR signal of the $\mathrm{Mn}^{\mathrm{III}} \mathrm{Mn}^{\mathrm{IV}}$ dimer and evidence of electron transfer between the $\mathrm{Mn}$ dimer core and the surface $\mathrm{Cr}$ centers by magnetic and optical spectroscopy, successful loading of a biomimetic catalyst on the nanopores was demonstrated. 
Magnetic control of spatial organization at bio-membranes

Principal Investigator: Jay T. Groves

Project description:

The spatial heterogeneity of cellular membranes impacts numerous biological processes. A large body of evidence points to cholesterol dependent phase separation as a key characteristic of membrane structure and organization. Comparatively little is known, however, about connections between membrane mechanics and cholesterol dependent spatial organization. The goal of this project was to seed a new set of experimental investigations into the coupling of membrane curvature to its phase structure and spatial organization.

\section{Accomplishments:}

As the title would imply, one of the envisioned experimental methodologies involved use of magnetic particles to manipulate vesicles. This was investigated in parallel with other strategies, and in the end we chose to work with curvature modulated surfaces instead. These can be fabricated by a combination of photolithographic patterning and controlled isotropic and anisotropic etching steps.

These curvature modulated substrates, along with the supported membrane systems we have previously developed proved exceedingly effective to study membrane curvature effects, and we have made several novel contributions using this system. First, we have demonstrated that curvature gradients are capable of driving the spatial organization of micron sized membrane domains. Second, we were able to quantify the strength of this effect, which provides for the first time an estimate of the amount of membrane deformation that would be required to move membrane structures in living cells. This seminal work has been published (Langmuir 2006, 22: 5347) and we have also written an invited review on this general area for Soft Matter (2007, in press).

The curvature patterning technique is rapidly growing into other project areas within our group. Curvature modulation is now being used to study cell membrane organization in living T cells, both here at LBNL and through a collaboration with Prof. R. Vale at UCSF. These concepts and basice experimental strategies will be finding their way into several new funding proposals. 


\section{Rapid Characterization of Microbial RNAs using Artificial Nanopores Principal Investigators: E. Trepagnier, A. Radenovic, Jan T. Liphardt}

\section{Project Description}

RNA molecules have numerous structural and functional roles in microbial molecular machines such as the ribosome. RNA is also an active component of viral machines such as the rotary motor of the $\phi 29$ bacteriophage. To understand how RNA catalyzes reactions and regulates biological processes, it is essential to understand the mechanical properties of large RNA assemblies, and to learn how RNA structure (e.g. helices, loops, $\mathrm{Mg}^{2+}$ binding sites) relates to RNA function (e.g. conversion of chemical potential stored in ATP to mechanical forces, catalysis of RNA cleavage and ligation).

We are developing a fundamentally new approach for determining RNA structure, dynamics, and responses to mechanical force that promises very high throughput. In principle, it should be possible to characterize a microbe's entire RNA structural repertoire within hours.

The fundamental idea is that the folding state of a single RNA molecule can be determined by pulling it through a limiting aperture - if the RNA is folded, the forces needed to pull it through the pore will be much larger (e.g. $15 \mathrm{pN}$ vs. $1 \mathrm{pN}$ ) than if it is unstructured. Practically, we tether single RNA molecules to a polystyrene bead, trap this bead in a laser trap, and bring the trapped bead into proximity of a $3 \mathrm{~nm}$ diameter artificial nanopore. We then thread the polymer through the pore with an external electrical potential, while simultaneously monitoring the progression of the polymer through the pore using the laser tweezers. In this way, it is possible to determine the relative position and stability of a RNA structure on long RNA transcripts.

\section{Accomplishments}

In last year's report, we demonstrated the feasibility of mechanical translocation of biopolymers through nanopores. Since then we have made progress in four areas, 1) the optimization of the attachment chemistry of the RNA to the beads, 2) reduction of background noise in the current measurements due to laser illumination of measurement electrodes, 3) the development of a microfluidics system that allows easy buffer exchange in the experimental flowchamber, and 4) we have also resolved the key bottleneck that greatly hindered progress, the limited availably of high quality nanopores.

Previously, we had been using an 8 step fabrication procedure for making the artificial nanopores. In this approach, focused-ion-beam thinned $\mathrm{SiN}$ membranes were drilled with an argon ion beam, and the pores were then closed by atomic layer deposition. Now, in collaboration with Ken Downing in the LBNL Life Sciences Division, we have succeeded in manufacturing the nanopores using a focused electron beam, which has greatly improved the numbers of nanopores available for our experiments.

In conclusion, we have now clearly demonstrated that the proposed approach can be implemented, and that the numerous technical challenges can be overcome. The next steps will be to characterize and improve the temporal and spatial resolution of this approach and find ways of handling very long polymers - at present we are limited to about 40 kilobases. Follow-up funding from three sources, including Searle and Sloan foundations, has been obtained. 


\section{Biomineral engineering for the production of photonic structures and light collection systems \\ Principal Investigator: Jan T. Liphardt}

\section{Project Description}

Inorganic scaffolding materials of desired nanostructure and chemical makeup are essential for enabling the development of solar to fuel systems. Specifically, custom silica and other inert oxide nanostructures are needed with selectively functionalized pores or channels. For the most promising designs, no synthetic methods are currently available for generating the corresponding functionalized oxide nanostructures. The general goal of this project is to develop a scalable technology for producing inexpensive and biocompatible complex, functionalized oxide nanostructures. This goal will be accomplished by engineering the natural biomineralization pathways of diatoms to produce custom nanostructures.

Diatoms and some marine sponges produce exquisitely complex photonic structures, including optical fibers and microlens systems. For example, the arctic sea sponge Rosella racovitzae synthesizes solar collection arrays composed of "spicules", which are single-mode optical fibers with transmission properties that rival commercial fibers.

The specific goals of this project are to learn how to dope biosilica structures with specific ligands that permit covalent attachment of dyes and catalysts, such as e.g. Mn complexes through phenolic bridges or oxo linkages.

\section{Accomplishments}

Our goal has been to learn how to store, grow, manipulate, and image diatoms and other producers of biomaterial structures. In collaboration with Jay Keasling and Kris Niyogi (both at LBNL), we have established a variety of diatom and dinoflagellate cultures (including $T$. pseudonana and C. longipes) at LBNL. T. pseudonana was chosen as one model system because it has a radially symmetric fully silicified cell wall with pores having a range of diameters. It has also been sequenced and a method for stable transformation of pseudonana and a series of expression vectors is now available. Therefore, both $T$. pseudonana can be investigated using the standard tools of functional genomics. C. longipes was chosen as the other model system since it is grows long fibers ( $>80$ microns) that have highly desirable optical properties, based on preliminary studies.

We have also been able to dope growing biosilica structures with fluorescent dyes. These doped biosilica structures have been analyzed with epi-fluorescence and confocal imaging, and we have now begun to compare the incorporation efficiencies of various dopants, including polyethyeneglycol (PEG) stabilized quantum dots. Finally, in collaboration with Fred Wilt (UC Berkeley), we have used atomic force microscopy to image the surface of calcium carbonate structures produced by sea urchins.

In aggregate, LBNL now possesses the capability for growing and staining diatoms and dinoflagellates, and characterizing biosilica structures using optical methods and atomic force microscopy. 
Structure and Function of Intact Cellulosomes

Principal Investigators: Michael A. Marletta and Jamie H. D. Cate

\section{Project Description}

Derived from solar energy, cellulose is the most abundant biomolecule on the planet and, therefore, could be used to generate renewable energy sources that might replace the use of oil. At present, there are no efficient or economical means for converting cellulose into useful biofuels. This is primarily due to the insoluble and heterogeneous nature of cellulose sources, i.e. plants. In the biosphere, cellulose in plant debris is efficiently degraded by some anaerobic microorganisms, which use the products for growth. It may, therefore, be possible to exploit the mechanisms used by these bacteria to break down cellulose to provide feedstock for biofuel production.

The molecular machinery responsible for the degradation of cellulose is found in supramolecular, multi-enzyme complexes called cellulosomes that can reach many MDa in size. Within the cellulosome, many degradative enzymes work synergistically to attack heterogeneous, insoluble cellulose substrates. Cellulosomes exist as extracellular complexes that are either attached to the surface of the bacteria, or are free in solution. These complexes differ between organisms, and even within the same organism may be heterogeneous. In some organisms, the cellulosomes are dependent on lipids for optimal activity. These challenges have greatly complicated efforts to probe cellulosome structure and function.

The goal of the present project is to develop an experimental system to determine mechanistically how cellulosomes efficiently degrade cellulose. At present, it has not been possible to probe the structure and function of intact cellulosomes, due to their very large size and heterogeneous composition in many organisms. Furthermore, since the substrate for cellulosomes is a solid, the cellulosome is not easily studied by standard enzymological approaches. We propose to develop a model system of the cellulosome that will enable us to study its enzymatic properties at a fundamental level, and to determine its structure at atomic resolution. The long-term benefit of this work will further an important goal of the DOE, to improve conversion of cellulose into useful liquid fuels, or biofuels.

\section{Accomplishments}

We have cultured a Clostridium species in the lab that produces soluble cellulosomes less than $1 \mathrm{MDa}$ in size. We are now characterizing the components and activity of these cellulosomes by various protein biochemical approaches, including mass spectrometry. We have also been optimizing growth conditions to improve yields of cellulosomes for crystallization trials. Finally, we have developed a transformation protocol for the Clostridium strain that will allow us to carry out genetic manipulations of the cellulosome for biophysical studies. 


\title{
Physics Division
}

LB05002

\author{
Advanced Monolithic Si Pixel Sensors \\ for the International Linear Collider \\ Principal Investigator: Marco Battaglia
}

The R\&D program addresses the development of Si pixel sensors of new concept for the detection of ionizing particles at the next planned particle collider, the International Linear Collider (ILC). ILC physics requirements push the vertex tracker specifications to new levels. Monolithic CMOS pixel detectors, the technology of choice for this program, integrating readout and data processing capabilities into the same silicon substrate used for detection offer unprecedented opportunities in terms of position resolution and readout speed within a minimal amount of material. The ILC beam parameters stress the importance of high pixellization and fast readout capabilities, physics requirements pushes towards ultrathin sensors with low power dissipation, to minimize the disturbance induced to the charged particle trajectories.

The second year of the project has been devoted to detector testing, specifications and design of a second chip, sensor back-thinning characterization and design of a beam telescope for the ALS. The response and radiation hardness of the first CMOS pixel sensor designed at LBNL and manufactured in FY05, named LDRD-1, has been characterized in details. Response to ionizing radiation has been studied with both a ${ }^{55} \mathrm{Fe}$ radioactive source and the $1.5 \mathrm{GeV} e^{-}$beam extracted from the ALS booster ring at the BTS. The sensor performed well on the beam and demonstrated that the technology chosen, AMS 0.35 $\mu \mathrm{m}$ OPTO, is radiation tolerant within the ILC specifications. Charge sharing has been studied using an highly collimated laser beam. Results show that a $20 \times 20 \mu \mathrm{m}$ pixel provides a single point resolution of $3 \mu \mathrm{m}$, in agreement with simulation. These results fix the pixel size for the second pixel structure, LDRD-2, which has been designed, submitted and received by the end of FY06. This new structure has $20 \times 20 \mu \mathrm{m}$ pixels and features advanced functionalities, including self-biased architecture, in-pixel signal and baseline storage and correlated double sampling. Minimizing the detector thickness is essential for preserving high tracking accuracy down to low-momenta, where multiple scattering dominates the track resolution. CMOS pixel sensors are especially well suited for manufacturing ultra-thin sensors since the active region is confined within approximately $30 \mu \mathrm{m}$. A comprehensive program of tests of sensor back-thinning feasibility, yield and performance has been carried out. Final results show that diced chips can be back-thinned to $40 \mu \mathrm{m}$ with good yields and no appreciable change in noise and response to minimum ionizing particles. The back-thinning program has been completed by the design and construction of a beam telescope prototype, based on three back-thinned CMOS pixel sensors and a reference thick detector, this is the first beam telescope based on thin CMOS pixel sensors ever built. The beam telescope has been deployed on the ALS $e^{-}$beam at the BTS and successfully operated. Preliminary results from analysis of these data show that three layers of thin CMOS pixel sensors provide a particle extrapolation accuracy matching ILC specifications. Detector design and testing has been complemented by significant progress in the development of a CMOS sensor signal simulation program, implemented in the ILC C++-based framework, which uses energy deposits from Geant-4 simulation and produces lcio objects, which can be handled by the ILC reconstruction software. The full chain from sensor simulation to cluster reconstruction, patter recognition and track fit is now available and allows, for the first time, to analyse lab and beam test data and physics simulation through the same algorithms, within the ILC $\mathrm{C}++$ framework. 


\section{Development of Nanowire Carpet Hybrid Pixel Detectors (NCHyPs) Principal Investigator(s): Maurice Garcia-Sciveres}

\section{Project Description}

The purpose of this project is to try to develop a new, more affordable fabrication method for hybrid pixel radiation imaging detectors, by exploiting nanotechnolgy techniques. A hybrid pixel detector is a combination of an electronics integrated circuit with pixellated a solid state sensor. These detectors are critical in high energy physics and synchrotron light source applications. The only presently available fabrication method uses area array bump bonding to interconnect the integrated circuit to the sensor. This is a very costly operation and additionally requires custom fabricated solid state sensors exactly matching the geometry of the integrated circuit (also very costly and prohibitive for low volume).

The NCHyP concept proposes a sensor that is universal (can be mated to any geometry integrated circuit) and does not require bump bonding for interconnection. The nanowires are like tiny hairs (100 nanometer diameter) that can be grown to cover the surface of a silicon wafer. Each nanowire is capped with a gold dot, and additionally forms a diode junction with the bulk of the wafer. Using nanowires this way circumvents the costly lithography, implantation and metalization steps needed to fabricate a sensor by conventional means. This project aims to exploit the gold coated tips of the wires to form the electro-mechanical contact to the integrated circuit (by either compression bonding or soldering) in order to avoid the costly bump bonding.

\section{Accomplishments}

This LDRD project was to study NCHyP circuit prototypes. The LDRD covers physics and engineering work to design and characterize these prototypes, the actual fabrication was done by the Molecular Foundry. The main accomplishments have been the production of mechanical silicon samples with nanowires and the tuning of the growth parameters, the modification of integrated circuits from the ATLAS pixel detector of the Large Hadron Collider for use in this project, and the production of sensor quality silicon wafers that will lead to functional NCHyP prototypes. A preliminary demonstration of electrical "dry contact" (no mechanical bond) between the nanowires and an integrated circuit has been achieved, but a characterization of the quality of such contact has still to follow. This type of contact can be used to study the electrical properties of NCHyP even before an electro-mechanical bonding technique is developed.

Follow-on work includes the testing of a tin soldering technique for the electromechanical contact, and the first attempt at doped nanowire growth on sensor quality silicon substrates. The Molecular Foundry will also support further advances by additional fabrications. 


\author{
Designing a Novel Reactor Neutrino Oscillation Experiment \\ for Measuring the Unknown Neutrino Mixing Angle $\theta_{13}$ \\ Principal Investigators: S.J. Freedman, K.M. Heeger, R.W. Kadel, K.-B. Luk
}

\title{
Project Description
}

The purpose of this project is to design and explore detector technology for a nextgeneration reactor neutrino oscillation experiment. Using multiple detectors placed at different distances from nuclear reactors and reactor antineutrinos an experiment can search for oscillation effects in the $v_{\mathrm{e}} \rightarrow v_{\mu, \tau}$ channel and make a precision measurement of the unknown neutrino mixing angle $\theta_{13}$. The discovery of subdominant oscillation and non-zero $\theta_{13}$ would have a profound impact in neutrino physics. The size of $\theta_{13}$ is critical for finding $\mathrm{CP}$ violation in the lepton sector and will define research in neutrino oscillation physics for the next decade and beyond. Neutrino oscillation and CP violation in the lepton sector may lead to leptogenesis and ultimately might explain the observed matter-antimatter asymmetry in the Universe.

Our primary goals are to select a suitable experimental site, perform design and engineering studies for future neutrino detectors, prototype detector components, and assess possible backgrounds to this precision neutrino measurement through simulation and environmental measurements. Mechanical and civil engineering studies are needed to demonstrate the technical feasibility of the experiment.

\section{Accomplishments}

Last February, groups in China, Hong Kong, Taiwan, Czech Republic, Russia, and the US formed an international collaboration to conduct this challenging program called Daya Bay, an underground neutrino experiment located at the Daya Bay nuclear power plant in China to determine $\sin ^{2} 2 \theta_{13}$ with a sensitivity of better than 0.01 . The nearby mountains of the site provide sufficient overburden to minimize the cosmogenic background in the underground detectors, and allow construction of horizontal tunnels to access the detectors. At LBNL we performed mechanical engineering work on the detector design as well as conceptual engineering studies on the layout for the underground laboratory. Scientific staff at LBNL performed detector simulations in support of engineering studies to characterize the physics potential and the ultimate sensitivity of the experiment. With the help from a tunnel engineer at Fermilab, Berkeley has also involved in the geotechnical survey of the site.

The Daya Bay proposal was reviewed by the P5 committee in last April and DOE in October. The committees endorsed the conceptual design of the project. A project document prepared by the Daya Bay collaboration is now available to the international community. In June, Daya Bay was one of the two major initiatives extensively discussed in the workshop for USChina cooperation in high-energy physics. In July, LBNL and IHEP of China signed a memorandum of understanding for collaboration, with Daya Bay as one of the collaborative efforts.

The R\&D activities of the project in the U.S. intensified after a preliminary award from DOE. In addition to contributions in simulation and mechanical engineering, Berkeley is now providing expertise in project management, computing and electronics design as well as scientific leadership to Daya Bay. 
New Directions for Theoretical Physics at the TeV-Scale Principal Investigator: Hitoshi Murayama

\section{Project Description}

The overall motivation for this project is to address new directions in theoretical physics that brings together somewhat disparate yet connected subfields, to construct fully consistent theoretical frameworks of TeV-scale physics.

Particle physicists have known since the time of Fermi (1933) that the TeV scale is an important energy scale in physics. Together with the energy scale of gravity, derived from Newton's constant, it is now viewed as one of the two fundamental energy scales of nature. Much of the development in particle physics has revolved around how we understand these two energy scales. At this time the theoretical understanding of the $\mathrm{TeV}$ scale is extremely poor. The only phenomenologically viable model at hand is the toy model called the Minimal Standard Model, which is obviously unsatisfactory because of its many theoretical shortcomings. Many speculations have been discussed; including such ideas as supersymmetry, large extra dimensions, warped extra dimension(s) and new strong interactions. While all of these ideas have strong theoretical appeal, and some have interesting connections to string theory, arguably the theory of everything, it is fair to say that none of them are free from problems, and their consequences have not been worked out in complete detail. Our goal then is to come up with a consistent theoretical framework that will be confronted with the anticipated beautiful data at the TeV scale from the Tevatron and the LHC

\section{Accomplishments}

Our goal can only be approached in a multi-faceted fashion, which brings together somewhat disparate yet connected subfields. To accomplish this, specific problems were addressed by different postdocs and students: Brian Freivogel has collaborated with Professor Raphael Bousso on three papers concerning the vacuum structure of string theory and its connection to the hypothesis of internal inflation. This line of reasoning implies that our observable universe is one of a vast number of many universes, the "multiverse," suggesting a possible anthropic explanation of many of the features of our universe. Eric Gimon has continued to investigate black holes in the context of string theory and supergravity. With Ferrara and Kalosh he has demonstrated and studied the properties of black hole solutions in $\mathrm{N}=8$ supergravity theories. Matthew Schwartz, with Lewandoski and Katz, has shown that AdS/CFT duality can be used to understand the spectrum and structure of tensor mesons in QCD. With (then) Divisional Fellow Christian Bauer, he showed that Soft Collinear Effective Theory can be used to analyze the properties of hadronic jets. Their work is the first step in a program to provide a systematically improvable treatment of jet properties, which is key to realizing the potential of the Large Hadron Collider to make and analyze new discoveries. 


\section{Cross-Divisional}

LB06031

Fabrication of photovoltaic devices using nanostructured biomaterials Principal Investigator(s): Seung-Wuk Lee

\section{Project Description}

The purpose of this project is to fabricate virus-based photovoltaic devices to harness solar energy. The monodisperse photovoltaic semiconductor nanowires will be synthesized through a mineralization process on genetically engineered viruses. Photovoltaic devices will be fabricated using semiconductor covered-virus nanowires through a conventional organicinorganic heterojunction photovoltaic solar cell fabrication process. The approach will exploit combinatorial phage display to identify specific binding peptide information against photovoltaic semiconductor materials $\left(\mathrm{CdTe}, \mathrm{ZnO}, \mathrm{TiO}_{2}\right.$, etc), which will direct mineralization of such materials in mild aqueous conditions. Using this tactic, we will engineer monodisperse viral particles to template photovoltaic semiconducting materials. These viruses will then be vertically self-assembled on transparent electrodes to fabricate solar cell devices.

We will develop virus-based organic-inorganic heterojunction photovoltaic devices, by exploiting viruses (M13 bacteriophage) engineered to have specific affinity between major coat protein (pVIII) and photovoltaic semiconducting nanomaterials. The biological approach to build the proposed photovoltaic solar cell materials and devices has many promising aspects: 1) we can isolate previously unexplored novel peptide information against photovoltaic semiconducting material. Using the identified peptide information, we can design novel organisms, microbes, and biomaterials, with which we can open new fields of synthetic biology and couple many conventional micro-fabrication approaches (such as, soft lithography, DPN lithography, microprinting, and microfluidics) to build novel devices through bottom up processes. 2) Using recombinant DNA techniques, we can tune the length of the virus and subsequent nanowire scales which is suitable for the optimized device fabrication. In addition, cloning nature of the biological materials can simplify preparation of the sample through a self-replicating amplification processes. 3) Due to the diamagnetic property of the virus, we can align them vertically between the electrodes using external magnetic filed in the heterojeunction devices, which is expected to enhance the efficiency of the photovoltaic devices by improving electron mobility. 4) Obtaining knowledge between biomolecules and electronic materials is critical for the design of novel nanoscale bio-electronic devices. 5) This multifaceted research project will provide graduate and undergraduate students with many opportunities to explore, integrate and educate the next generation of scientists in a variety of diverse research disciplines.

Accomlishments

We have been established the reproducible protocol for incorporating desired peptide motifs on the pVIII surface protein coats of the M13 viruses. Our method uses recombinant DNA technologies to engineer the M13 phage genome. First we designed the DNA insert flanked with cloning site DNA sequences (PstI restriction enzyme sites). After making polymerase chain reaction product with the DNA inserts, we lysised them using PstI restriction enzyme, then lygased to construct the circular DNA. After transformation of the circular DNA, we could construct M13 phage viruses with specific desired peptides inserts. Using this approach, we already incorporated with four glutamic acids sequences onto phage major coat proteins, which we believed to nucleate various inorganic nanocrystals in non-specific manners.

We are in the process of applying these recombinant DNA protocols to construct major coat library of M13 bacteriophage to screen photovoltaic semiconductor specific peptide motifs. 


\section{Development and Application of Quantum Monte Carlo (QMC) Methods to biological Systems}

\section{Principal Investigators: William A. Lester, Jr., Graham Fleming, Michael Frenklach}

\section{Project Description}

The purpose of this research is to calculate accurately electronic excitations in biological and other large molecular systems using the quantum Monte Carlo (QMC) method. The highest priority is the application of the QMC method to the study of singlet-excited states and their role in energy transfer in photosynthetic systems. Significant progress has been made in reducing the computational effort with system size without loss of accuracy that makes possible the promise of application of the method to molecules containing more than 300 electrons. At present there is no other first-principles quantum-chemical method that can be applied to such large systems. In addition, there is the need to extend understanding of the behavior of these systems in solvents and embedded in other media.

\section{Accomplishments}

Several essential enhancements to our Zori QMC code have been achieved during the year. These advances include the introduction, optimization, and validation of effective core potentials. In addition, we introduced optimized linear algebra libraries into the evaluation of the trial wave function. The most significant development was the construction of a linear-scaling version of the Schmidt-Moskowitz correlation function that is more than an order of magnitude faster than the original implementation. The faster code made apparent the need to address numerical stability of random walks. We have added routines to eliminate statistical outliers from averages and to reduce the branching step of DMC calculations. Error handling of problematic walkers has increased robustness of Zori that is important for massively parallel computations. We have refined the restart capability of the code to be portable across parallel computing platforms.

Calculation of the photosynthetic rate proceeds through calculation of an energy transfer matrix element. In the past year several new routines have been incorporated in Zori to compute offdiagonal contributions. Testing the approach for evaluation of the requisite terms continues.

A number of approaches for the calculation of energies in the presence of media were investigated. In these schemes, the active region is treated by $\mathrm{DMC}$, and the surrounding region is addressed either by VMC or by empirical approaches. Research in this direction continues. 


\section{Publications List}

AFRD-Byrd LB06003 High-Stability Beam Handling in Electron Storage Rings with Applications to High Energy Colliders and Light Sources

S. De Santis, A. Wolski, M.C. Ross; "Design Of A Fast Extraction Kicker For The Accelerator Test Facility," 2006 European Particle Accelerator Conference (June 2006). http://accelconf.web.cern.ch/AccelConf/e06/PAPERS/THPLS113.PDF

C. Steier, Walter Barry, J. M. Byrd, F. Sannibale, T. Scarvie, A. Wolski; "Experimental Studies of the Fast Ion Instability at the ALS," submitted to the 2007 Particle Accelerator Conference (June 2007).

AFRD-Lidia LB04019 Critical Accelerator Technologies for Advanced Light Sources

J. Byrd, L. Doolittle, A. Ratti, J. W. Staples, \& R. Wilcox; “Timing Distribution in Accelerators via Stabilized Optical Fiber Links," Proceedings of the 2006 International Linear Accelerator Conference, Knoxville, Tennessee.

R. Wilcox and J. Staples; "Syncnronizing Lasers Over Fiber by Transmitting Continuous Waves," submitted to Conference on Lasers and Electro Optics 2007.

M. Gullans, J. Wurtele, G. Penn, \& A. Zholents; "Performance Study of a Soft X-ray Harmonic Generation FEL Seeded with an EUV Laser Pulse," LBNL-61592.

M. Gullans, J. Wurtele, G. Penn, \& A. Zholents; "A source of coherent soft x-ray radiation based on high-order harmonic generation and free electron lasers," Proceedings of EPAC 2006, Edinburgh, Scotland.

G. Penn, M. Reinsch, \& J. Wurtele; "Analytic model of bunched beams for harmonic generation in the low-gain free electron laser regime," Phys. Rev. ST Accel. Beams 9, 060702 (2006).

W. Fawley, G. Penn, \& A. Zholents; "Output Bandwidth Effects in Seeded, Harmonic Cascade FELs," LBNL-60156.

W. M. Fawley, “An Enhanced GINGER Simulation Code with Harmonic Emission and HDF5 IO Capabilities," LBNL-60155.

J. Staples, S. Lidia, F. Sannibale, \& S. Virostek; "Design of a VHF-band RF Photoinjector with MegaHertz Beam Repetition Rate,” LBNL-62111.

AFRD-Logan LB05022 Enabling High Energy Density Physics at LBNL

\section{Refereed Publications}

J.J. Barnard, J. Armijo, R.M. More, A. Friedman, I. Kaganovich, B.G. Logan, M.M. Marinak, G.E. Penn, A.B. Sefkow, P. Santhanam, D.R. Welch, J.S. Wurtele; "Theory and simulation of warm dense matter targets," Nuclear Instruments and Methods in Physics Research A, accepted for publication (2006), HIFAN 1523, LBNL-59851.

F.M. Bieniosek, J. Barnard, M.A. Leitner, A.W. Molvik, R. More, R.K. Roy; "Diagnostics for Near Term WDM Experiments," Nuclear Instruments and Methods in Physics Research A, accepted for publication (2006), HIFAN 1519, LBNL-59797.

R. More, H. Yoneda, H. Morikami; "Short-pulse lasers and electron dynamics in warm dense matter," JQSRT 99,409 (2006).

H. Yoneda, H. Morikami, K.-I. Ueda and R. More; "Ultra-short pulse laser pump-probe experiments for investigation of warm dense plasmas," JQSRT 99, 690 (2006). 
L.R. Grisham, J.W. Kwan, G. Westenskow; "Halogens for Negative Ion Beams and Ion-Ion Plasmas, " Nuclear Instruments and Methods in Physics Research A, accepted for publication (2006), HIFAN 1469, LBNL-61290.

B.G. Logan, F.M. Bieniosek, C.M. Celata, J. Coleman, J., et al; "Recent U.S. advances in ion-beam-driven high energy physics and heavy ion fusion," Nuclear Instruments and Methods in Physics Research A, accepted for publication (2006), HIFAN 1466, LBNL60980.

P. Michel, E. Esarey, C.B. Schroeder, B.A. Shadwick, and W.P. Leemans; "Efficient electron injection into plasma waves using higher-order laser modes," Phys. Plasmas 13, 113112 (2006), LBNL-62022.

W.P. Leemans, B. Nagler, A.J. Gonsalves, Cs. Toth, K. Nakamura, C.G.R. Geddes, E. Esarey, C.B. Schroeder, and S.M. Hooker; "GeV electron beams from a centimetre-scale accelerator," Nature Phys. 2, 696-699 (Oct 2006), LBNL-60105.

P. Michel, C.B. Schroeder, B.A. Shadwick, E. Esarey, and W.P. Leemans; "Radiative damping and electron beam dynamics in plasma-based accelerators," Phys. Rev. E 74, 026501 (2006), LBNL-59205.

G. Fubiani, J. Qiang, E. Esarey, W. P. Leemans, and G. Dugan; "Space charge modeling of dense electron beams with large energy spreads," Phys. Rev. ST Accel. Beams 9, 064402 (2006), LBNL-60089.

J. van Tilborg, C. B. Schroeder, C. V. Filip, Cs. Toth, C. G. R. Geddes, G. Fubiani, E. Esarey, and W. P. Leemans; "Terahertz radiation as a bunch diagnostic for laserwakefield-accelerated electron bunches," invited paper, Phys. Plasmas 13, 056704 (2006), LBNL-60017.

C. B. Schroeder, E. Esarey, B. A. Shadwick, and W. P. Leemans; "Trapping and dark current in nonlinear plasma waves," Phys. Plasmas 13, 033103 (2006), LBNL-58353.

G. Fubiani, E. Esarey, C. B. Schroeder, and W. P. Leemans; "Improvement of electron beam quality in optical injection schemes using negative plasma density gradients," Phys. Rev. $E$ 73, 026402 (2006), LBNL-58354.

C.V. Filip, Cs. Toth and W.P. Leemans; " Optical cross-correlator based on supercontinuum generation," Optics Express 14, 2512-2519 (2006).

J. van Tilborg, C. B. Schroeder, C. V. Filip, Cs. Toth, C. G. R. Geddes, G. Fubiani, R. Huber, R. A. Kaindl, E. Esarey, and W. P. Leemans; "Temporal Characterization of Femtosecond Laser-Plasma Accelerated Electron Bunches using THz Radiation," Phys. Rev. Lett. 96, 014801 (2006), LBNL-58351.

W. Leemans, E. Esarey, C. Geddes, C. Schroeder, Cs. Toth; "Laser Guiding for GeV LaserPlasma Accelerators," Philos. Trans. R. Soc. London, Ser. A 364, 585-600 (2006), LBNL58352.

\section{Invited Talks}

S.S. Yu, B.G. Logan, J.J. Barnard, F.M. Bieniosek, et al; "Heavy-Ion-Fusion-Science: Overview of U.S. Progress," invited paper presented at the IAEA in China October 2006, HIFAN 1438, LBNL-59441.

W. Leemans, "Characterization of laser accelerated electron beams with coherent THz," invited talk at 12th Beam Instrumentation Workshop, Batavia, IL, May 1 - 4, 2006.

E. Esarey, "Recent Progress on Laser-Plasma Accelerators," distinguished plenary talk at 12th Advanced Accelerator Concepts Workshop, Lake Geneva WI, 10-15 July 2006.

E. Esarey, "High-Quality GeV-Level Electron Beams from Laser Plasma Accelerators," invited talk at LINAC 2006, Knoxville TN, 21-25 Aug 2006. 
E. Esarey, "Laser-driven wavebreaking, electron trapping, and mono-energetic beam production," invited talk at 48th APS Division of Plasma Physics Meeting, Philadelphia PA, Oct 30-Nov 3, 2006.

W.P. Leemans, "GeV electron beams from cm-scale laser driven plasma based accelerators," invited talk at 48th APS Division of Plasma Physics Meeting, Philadelphia PA, Oct 30Nov 3, 2006.

C.G.R. Geddes, "Developing high energy, stable laser wakefield accelerators: particle simulations and experiments," invited talk at 48th APS Division of Plasma Physics Meeting, Philadelphia PA, Oct 30-Nov 3, 2006.

\section{AFRD-Zolotorev LB05039 Low Energy Spread Electron Source}

M. Zolotorev, E. D. Commins, S. Heifets and F.Sannibale; "Proposal for a High-Brightness Pulsed Electron Source," to be published in proceedings of the workshop on The Physics and Applications of High Brightness Electron Beams, Erice, Italy, October 2005. LBNL60066. http://www.physics.ucla.edu/PAHBEB2005/program/index.htm

M. Zolotorev, E. D. Commins, and F. Sannibale; "Quantum-degenerate electron source: a proposal," submitted to Physical Review Letters.

M. Zolotorev, E. D. Commins, and F. Sannibale; "Theory and proposal for a quantumdegenerate electron source," LBNL Formal Report LBNL-61587. Berkeley, September $11,2006$.

M. Zolotorev, E. D. Commins, and F. Sannibale; "Quantum-degenerate electron source," Physics Department seminar at the University of California of Berkeley, September 26, 2006.

During FY06 an application for provisional patent has been filed.

\section{ALS-Howells LB04013 Coherent X-Ray Diffraction Imaging (CXDI)}

Barty, A., S. Marchesini, H. N. Chapman, M. R. Howells, C. Cui, A. Minor, J. C.H.Spence, U. Weierstall, A. Noy, B. Artyukhin, T. Baumann, T. Willey, T. v. Buuren, J. Stolken, J. H. Kinney; "Three-dimensional ceramic nanofoam lattice structure determination using coherent X-ray diffraction imaging: insights into deformation mechanisms," submitted to Nature Materials.

Chapman, H. N., A. Barty, M. J. Bogan, S. Boutet, et al; "Femtosecond diffractive imaging with a soft-X-ray free-electron laser," Nature Physics 2, 839-843 (2006a).

Chapman, H. N., A. Barty, S. Marchesini, A. Noy, S. H.-. Riege, C. Cui, M. R. Howells, R. Rosen, H. He, J. C. H. Spence, U. Weierstall, T. Beetz, C. Jacobsen, D. Shapiro; "Highresolution ab initio Three-dimensional X-ray Diffraction Microscopy," Journal of the Optical Society of America A 23, 1179-1200 (2006b).

Marchesini, S., H. N. Chapman, A. Barty, C. Cui, M. R. Howells, J. C. H. Spence, U. Weierstall, A. M. Minor; "Phase Aberrations in Diffraction Microscopy," in Proceedings of the 8th International Conference on X-ray Microscopy, Himeji, Japan; Vol. 7, IPAP Conf. Series (2006a).

Marchesini, S., H. N. Chapman, A. Barty, A. Noy, S. P. Hau-Riege, J. M. Kinney, C. Cui, M. R. Howells, R. Rosen, J. C. H. Spence, U. Weierstall, D. Shapiro, T. Beetz, C. Jacobsen, E. Lima, A. M. Minor, H. He; "Progress in Three-Dimensional Coherent X-Ray Diffraction Imaging," in Proceedings of the 8th International Conference on X-ray Microscopy, Himeji, Japan; Vol. 7, IPAP Conf. Series (2006b). 
ALS-Kirz LB04044 Lenless Imaging of Yeast Cells

E. Lima, "The Advancement of Biological Imaging Through X-ray Diffraction Microscopy, "Ph.D. thesis, Stony Brook University, August 2006 http://xray1.physics.sunysb.edu/ micros/publications/dissertations/lima_phd_2006.pdf

ALS-Martin LB05024 Left-Handed Nanoscale Meta-Materials: Towards the Optical Domain

Zhao Hao, Michael C. Martin, Bruce Harteneck and Alex Liddle; "Nano-scale Metamaterials: Fabrication and Optical Measurements from THz towards visible," IRMMW-THz2006, Shanghai, China, IEEE 06EX1385 (September 2006).

Zhao Hao, Michael C. Martin, J. Alexandre Liddle, and Bruce Harteneck; "Strong Broadband Absorption and Reflection from Double Split Ring Magnetic Resonators," in preparation for possible submission to Phys. Rev. Lett.

Zhao Hao, Michael C. Martin, Erik Anderson, Stefano Cabrini, J. Alexandre Liddle, and Bruce Harteneck; "Direct Magnetic Resonances with Infrared Light from Plasmonic Single Closed Ring Resonators," in preparation for possible submission to Phys. Rev. B.

ALS-Scholl LB04030 Ultrafast Magnetization Dynamics

A. F. Bartelt, A. Comin, J. Feng, J. R. Nasiatka, T. Eimüller, B. Ludescher, G. Schütz, H. A. Padmore, A. T. Young, A. Scholl; "Element-specific Spin and Orbital Moment Dynamics of Fe/Gd Multilayers," submitted to Applied Physics Letters.

CH-Ahmed LB04038 Gas Phase Studies of the Building Blocks of Life

K. R. Wilson, D. S. Peterka, M. Jimenez-Cruz, S.R. Leone, and M. Ahmed; "VUV Photoelectron Imaging of Biological Nanoparticles - Ionization energy determination of nano-phase glycine and phenylalanine-glycine-glycine," Phys. Chem. Chem. Phys. 8, 1884 (2006). LBNL-59291.

K. R. Wilson, L. Belau, C. Nicolas, M. Jimenez-Cruz, S. R. Leone, and M. Ahmed; "Direct determination of the ionization energy of histidine with VUV synchrotron radiation," Int. J. Mass Spectrom. 249-250, 155, (2006). LBNL-58989.

K. R. Wilson, M. Jimenez-Cruz, C. Nicolas, L. Belau, S. R. Leone, and M. Ahmed; "Thermal Vaporization of Biological Nanoparticles: Fragment-Free VUV Photoionization Mass Spectra of Tryptophan, Phenylalanine-Glycine-Glycine and $\beta$-carotene," J. Phys. Chem. $A$ 110, 2106 (2006). LBNL-58668.

M. Ahmed, "Photoionization of desorbed neutrals from surfaces," Encyclopedia of Mass Spectrometry, Vol. 6, Elseiver (in press). LBNL-60001.

L. Belau, K. R. Wilson, S. R. Leone, and M. Ahmed; "Vacuum-Ultraviolet (VUV) photoionization studies of the micro-hydration of DNA bases (Guanine, Cytosine, Adenine and Thymine)," to be submitted to J. Chem. Phys.

L. Belau, K. R. Wilson, S. R. Leone, and M. Ahmed; "Vacuum Ultraviolet (VUV) photoionization of small water clusters," to be submitted to J. Am. Chem. Soc.

L. Belau, K. R. Wilson, S. R. Leone, and M. Ahmed; "VUV post-ionization of laser desorbed organic surfaces," to be submitted to Anal. Chem. 
CH-Leone LB05019 Time-resolved X-ray Absorption Spectroscopy of Metalloporphyrins

Z.-H. Loh, M. Khalil, R. E. Correa, R. Santra, and S. R. Leone; "Orbital alignment in optical field-ionized Xe probed by femtosecond extreme ultraviolet absorption spectroscopy," in preparation for submission to Phys. Rev. Lett. or Phys. Rev. A.

CH-Neumark LB04027 Spectroscopy and Dynamics of Pure and Doped Helium Nanodroplets

J. H. Kim, D. S. Peterka, C. C. Wang, and D. M. Neumark; "Photoionization of helium nanodroplets doped with rare gas atoms," J. Chem. Phys. 124, 214301 (2006).

D. S. Peterka, J. H. Kim, C. C. Wang, and D. M. Neumark; "Photoionization and photofragmentation of $\mathrm{SF}_{6}$ in helium nanodroplets," J. Phys. Chem. B 110, 19945 (2006).

CH-Prausnitz LB06027 Properties of New Ionic Liquids for Electrochemical Applications and for Extraction of Heavy-Metal Cations from Wastewaters

N. Papaiconomou, J. Salminen, N. Yakelis and J. Prausnitz; "Synthesis and PhysicoChemical Properties of Ionic Liquids Containing Tetrakis(perfluorophenyl)borate, Tetraphenylborate and Trifluorophenylborate Anions," LBNL Report, to be submitted to J. Chem. Eng. Data.

N. Papaiconomou, N. Yakelis, J. Salminen, R. Bergman and J. Prausnitz; "Synthesis and Properties of Seven Ionic Liquids Containing 1-Methyl-3octylimidazolium or 1-Butyl-4methylpyridinium Cations," J. Chem. Eng. Data 51, 1389-1393 (2006).

J. Salminen, J. Prausnitz and J. Newman' "Studies of Ionic Liquids in Lithium-Ion Battery Test Systems," ECS Transactions, 1(26) 107-118 (2006).

J. Salminen, N. Papaiconomou, JM Lee and J Prausnitz; "Physico-Chemical Properties of Hydrophobic Ionic Liquids Containing 1-octylpyridinium, 1-octyl-2-methylpyridinium or 1-octyl-4-methylpyridinium Cations," J. Chem. Eng. Data, accepted for publication.

Y. Qin and J. Prausnitz; "Solubilities in ionic liquids and molten salts from a simple perturbed-hard-sphere theory," Ind. Eng. Chem. Res. 45, 5518-5523 (2006).

CH-Rescigno LB04029 Advanced Computational Methods for Photon-Molecule Collision Processes

W. Vanroose, F. Martín, T. N. Rescigno and C. W. McCurdy; "Complete Photofragmentation of the $\mathrm{H}_{2}$ Molecule as a Probe of Molecular Electron Correlation," Science 310, 1787 (2005).

F. Martín, D. A. Horner, W. Vanroose, T. N. Rescigno and C. W. McCurdy; "First Principles Calculations of the Double Photoionization of Atoms and Molecules using Bsplines and Exterior Complex Scaling," Proceedings of the Thirteenth International Symposium on Ionization, Correlation and Polarization in Atomic Collisions, Buenos Aires 2005; edited by A. Lahman-Bennani and B. Lohmann (American Institute of Physics AIP Conf. Proc. 811, 132 (2006)).

W. Vanroose, D. A. Horner, F. Martín, T. N. Rescigno and C. W. McCurdy, "Double Photoionization of Aligned Molecular, "Phys. Rev. A 74, 052702 (2006).

T. N. Rescigno, Wim Vanroose, D. A. Horner, F. Martín and C. W. McCurdy; "First Principles Study of Double Photoionization of $\mathrm{H}_{2}$ Using Exterior Complex Scaling," $J$. Elec. Spectros., Rel. Phenom. accepted for publication. 
D. A. Horner, W. Vanroose, T. N. Rescigno, F. Martin and C. W. McCurdy, "The Role of Nuclear Motion in the Photo-Double Ionization of Molecular Hydrogen," submitted for publication to Phys. Rev. Lett.

CH-Tilley LB05037 New Approach for the Catalytic Conversion of Methane and Other Inert Hydrocarbons

P. Bazinet, K. A. Tupper and T. D. Tilley; "Octa- and Nonamethylfluorene and an ElectronRich Permethylfluorenyl Ruthenocene Derivative," Organometallics 25, 4286 (April 18, 2006).

CS-Chevassut LB05008 Cryptographic Foundations for New Generation Distributed Systems

O. Chevassut, P. A. Fouque, P. Gaudry, D. Pointcheval; "The Twist-Augmented Technique for Key Exchange," International Workshop on Practice and Theory in Public Key Cryptography (PKC 2006), April 2006. LBNL-54709.

M. Abdalla, E. Bresson, O. Chevassut, and D. Pointcheval; "Password-based Group Key Exchange in a Constant Number of Rounds," International Workshop on Practice and Theory in Public Key Cryptography (PKC 2006), April 2006. LBNL-59542.

E. Bresson, O. Chevassut, D. Pointcheval; "A Security Solution for IEEE 802.11's Ad hoc Mode: Password-Authentication and Group Diffie-Hellman Key Exchange," International Journal of Wireless and Mobile Computing - Special Issue on Security of Computer Network and Mobile Systems, 2006. LBNL-57432.

M. Abdalla, O. Chevassut, B. Moeller, and D. Pointcheval; "Provably-Secure PasswordBased Authentication in TLS," Proceedings of the ACM Symposium on InformAtion, Computer and Communications Security (ASIACCS'06), March 2006. LBNL-57609.

M. Abdalla, O. Chevassut, B. Moeller, and D. Pointcheval; "Strong Password-Based Authentication in TLS Using the Three-Party Group Diffie-Hellman Protocol," International Journal of Security and Networks (IJSN), accepted for publication. LBNL59947.

CS-Markowitz LB06015 Integrated Microbial Community Genomes Data Management System

\section{Publications}

Markowitz, V.M. et al; “ An Experimental Metagenome Data Management and Analysis System," Bioinformatics, 22(14), 359-367 (2006). See also: http://img.jgi.doe.gov/m.

Martin, H.G. et al; "Metagenomic analysis of phosphorus removing sludge communities," Nature Biotechnology, 24(10), 1263-1269 (2006).

Woyke, T. et al; "Symbiosis insights through metagenomic analysis of a microbial consortium," Nature 443, 950-955 (2006).

\section{Presentations of the Metagenome Data Management and Analysis Tools}

Feb 2006 Metagenome Data Management and Analysis: The IMG/M Experimental System, Keystone Symposia on Microbial Community Genomics, Tahoe City, US.

July 2006 Metagenome Data, Management and Analysis, Metagenomics : Challenges and

Applications, National Academy of Sciences Panel, Washington D.C., US.

Aug 2006 An Experimental Metagenome Data Management and Analysis System, $14^{\text {th }}$

International Conference on Intelligent Systems for Molecular Biology (ISMB),

Fortaleza, Brazil. 
Aug 2006 An Experimental Metagenome Data, Management and Analysis System, $11^{\text {th }}$ International Symposium on Microbial Ecology (ISME), Vienna, Austria.

Oct 2006 Towards a Production Metagenome Data Management and Analysis System, $1^{\text {st }}$ International Conference on Metagenomics, San Diego, La Jolla, US.

CS-Oliker LB05029 Enhancing Commodity Scalar Processors with Vector Components for Increased Scientific Productivity

S.Williams, J. Shalf, L. Oliker, P. Husbands, S. Kamil, K. Yelick; "The Potential of the CELL Processor for Scientific Computing," ACM International Conference on Computing Frontiers, Ischia, Italy, May 7-9, 2007.

S. Kamil, K. Datta, S, Williams, L. Oliker, J. Shalf, K. Yelick; "Implicit and Explicit Optimizations for Stencil Computations," Memory Systems Performance and Correctness (MSPC), San Jose, California, Oct 222006.

J. Gebis, D. Patterson; "Embracing and Extending 20th Century Instruction Set Architecture: A Vector Case Study," IEEE Computer, in press.

CS-Pinar LB05031 Advanced Computational Tools for Electric Power Systems

V. Donde, V. Lopez, B. Lesieutre, A. Pinar and C. Yang, and J. Meza; "Identification of Severe Multiple Contingencies in Electric Power Systems," submitted to IEEE Transactions on Power Systems.

B. Lesieutre, S. Roy, V. Donde and A. Pinar; "Power Sytem Extreme Event Screening using Graph Partitioning," Proceedings of the 38th North American Power Symposium, 2006.

A. Pinar, V.Donde, B. Lesieutre, J. Meza, Y. Fogel; "Vulnerability Analysis of the Electric Power Grid," draft to be submitted to SIAM Optimization.

A. Pinar, A. Reichert, B. Lesieutre; "Computing Criticality of Lines in Power Systems," submitted to 2007 IEEE International Symposium on Circuits and Systems.

CS-Romano LB05032 Statistical Feature Modeling for Scientific Data Via Basis

Decomposition

R. Romano, C. Aragon, and Chris Ding; "Supernova Recognition using Support Vector Machines," Proceedings of the 5th International Conference of Machine Learning Applications, Orlando FL, December 14-16, 2006.

R. Ronano; Supernova Recognition using Support Vector Machines," invited talk for Neyman Seminar, Department of Statistics, UC Berkeley, September 20, 2006.

"Scientific Data Analysis via Statistical Learning," poster at Supercomputing 2006, Orlando FL, November 11-17, 2006.

CS-Wilkening LB06026 Extended First Order System Least Squares Finite Elements

J. Wilkening' "Stable asymptotics for corner singularities of elliptic systems," draft to be submitted to Comm. Pure. Appl. Math. 
ESD-Coates LB06004 An Investigation of the Microbial Processes Involved in Electron Transfer onto the Anode of a Biological Fuel Cell

\section{Papers}

J.C. Thrash, J.I. Van Trump, K.A. Weber, E. Miller, L.A. Achenbach, and J.D. Coates; "Electrochemical stimulation of microbial perchlorate reduction," Environmental Science and Technology (in press).

\section{Abstracts and poster presentations \\ K. Wrighton, P. Agbo, L.A. Achenbach, and J.D. Coates; "Geobacillus strain S2E, a novel thermophilic Fe(III)-reducer isolated from a thermophilic microbial fuel cell, "in abstracts of the 107th General Meeting of the American Society for Microbiology (2007). \\ J. C. Thrash, L.A. Achenbach, J.D. Coates; "Mediatorless Bioelectrical Perchlorate Reduction," in abstracts of the 107th General Meeting of the American Society for Microbiology (2006).}

J.C. Thrash, K.A. Weber, L.A. Achenbach, J.D. Coates; “A Novel Reactor for Bioelectrochemical Perchlorate Reduction," in abstracts of the 2006 SERDP Partners in Environmental Technology Technical Symposium \& Workshop (2006).

J. C. Thrash, K.A. Weber, J.D. Coates; "Humic Substances and Their Analogs for Electrochemical Stimulation of Microbial Perchlorate Reduction," in abstracts of the $13^{\text {th }}$ Meeting of the International Humic Substances Society (2006).

J. C. Thrash, K.A. Weber, L.A. Achenbach, J.D. Coates, "Electrochemical Stimulation of Microbial Perchlorate Reduction," in abstracts of the $106^{\text {th }}$ General Meeting American Society for Microbiology (2006).

J. C. Thrash, K.A. Weber, L.A. Achenbach, J.D. Coates; "Electrochemical Stimulation of Microbial Perchlorate Reduction," in abstracts of the 2005 SERDP Partners in Environmental Technology Technical Symposium \& Workshop (2005).

\section{Patent Submission}

J.D. Coates and J.C. Thrash; "Bioelectrical treatment of perchlorate and chlorate," patent pending, application number 60/867393 (2006).

ESD-DePaolo LB05011 Micro-characterization and Chemical Micro-dynamics of Atmospheric Mineral Dust

G. Lu, D. DePaolo, Q. Kang, and D. Zhang; "Lattice Boltzmann Simulation of Water Isotope Fractionation during growth of Ice crystals in clouds," Eos Trans. AGU, 87(52), Fall Meet. Suppl., Abstract H54D-08 (2006).

J.N.Christensen, , S.S. Cliff, R.A. VanCuren, L. Tomutsa, K.D. Perry, D.J. DePaolo; "Tracking Intercontinantal dust transport with radiogenic isotopes: Hefei, China to California," Eos Trans. AGU, 87(52), Fall Meet. Suppl., Abstract A43B-0138 (Spring 2006).

ESD-Gilbert LB05003 Behavior and Impact of Nanoparticles

B. Gilbert and J. F. Banfield; "Molecular-scale processes involving nanoparticulate minerals in biogeochemical systems," Reviews in Mineralogy and Geochemistry 59, 109-155 (2005) 
H. Zhang, B. Chen, B. Gilbert, and J.F. Banfield; "Kinetically controlled formation of a novel nanoparticulate $\mathrm{ZnS}$ with mixed cubic and hexagonal stacking," J. Mater. Chem. 16, 249-254 (2006).

B. Gilbert, F. Huang, Z. Lin, C. Goodell, H. Zhang, and J. F. Banfield; "Surface chemistry controls crystallinity of ZnS nanoparticles," Nanoletters 6, 605-610 (2006).

Z. Lin, B. Gilbert, Q. Liu, F. Huang; "A thermodynamically stable nanophase material," Journal of the American Chemical Society 128, 6126-6131 (2006).

B. Gilbert, H. Zhang, B. Chen, M. Kunz, F. Huang, and J. F. Banfield; "The compressibility of zinc sulfide nanoparticles," Physical Review B 74, 115405 (2006).

B. Gilbert, C. S. Kim, C.-L. Dong, J. Guo, P. S. Nico and D. K. Shuh; "Oxygen K-edge emission and absorption spectroscopy of iron oxyhydroxide nanoparticles," AIP Conference Proceedings: XAFS13 in press.

B. Gilbert, G. Lu and C. S. Kim; "Stable nanoparticle clusters formed under environmentally relevant conditions," submitted to Journal of Colloid and Interface Science.

B. Chen, B. Gilbert, H. Zhang and J. F. Banfield; "Mechanism of nanoparticle growth and phase transformation inhibition by surface impurities," submitted to Physical Review Letters.

J. Cervini-Silva, B. Gilbert, S. Fakra, S. Freidlich and J. F. Banfield; "Decarboxylation and polymerization of catechol and formation of $\mathrm{CeO}_{2}$ due to coupled redox and dissolution reactions at the surface of cerium(III) phosphate," Submitted to Geochim. et Cosmochim. Acta.

ESD-Miller LB04025 The California Water and Energy System: An Approach for Addressing Future Crises

Jin, J., and N. L. Miller; "Analysis of the impact of snow on daily weather variability in mountainous regions," Journal of Hydrometerology (in press).

Jin, J., and N. L. Miller; "Interactions between the ground water table and climate over the Merced watershed," submitted to Geophys. Res. Lett.

Pan, L., J. Jin, N.L. Miller, Y-S Wu, and G.S. Bodvarsson; "Coupling TOUGH2 with CLM3: Developing a coupled land surface and subsurface model," Vadose Zone Journal (in press).

Joyce, B., S. Vicuna, L. Dale, J. Dracup, M. Hanemann, and D. Purkey; "Climate Change Impacts on Water for Agriculture in California: A case study in the Sacramento Valley," submitted to Climatic Change. http://www.energy.ca.gov/2005publications/CEC-5002005-194/CEC-500-2005-194-SD.PDF

Leonardson, R., S. Vicuna, L..Dale and J. Dracup; "On the Accuracy of Snowmelt Runoff Predictions," submitted to Climatic Change.

ESD-Oldenburg LB06018 Coupled Modeling of Hydrology, Nutrient Cycling, and Vegetation: Applications to Water Quality and Water Balance

L. Pan, J. Jin, N.L. Miller, Y-S Wu, and G.S. Bodvarsson; "Modeling Hydraulic Responses to Meteorological Forcing: From Canopy to Aquifer," submitted to Vadose Zone Journal.

T. Xu, "Incorporation of Aqueous Reaction Kinetics and Biodegradation into TOUGHREACT: Application of a Multi-region Model to HydroBiogeoChemical Transport of Denitrification and Sulfate Reduction," submitted to Vadose Zone Journal. 
ESD-Steefel LB05036 Biogeochemical Reaction Rates and Pathways in Porous Media

Maher, K., C.I. Steefel, D. DePaolo, B. Viani; "The mineral dissolution rate conundrum: Insights from reactive transport modeling of $U$ isotopes and pore fluid chemistry in marine sediments," Geochimica et Cosmochimica Acta 70: 337-363 (2006).

Steefel, C.I, D. DePaolo, and P.C. Lichtner; "Reactive transport modeling: An essential tool and a new research approach for the Earth sciences," Earth and Planetary Science Letters 240: 539-558 (2005).

Li, L., C.I. Steefel, L. Yang; "Mineral dissolution kinetics in a single pore," to be submitted to Science.

Yang, Li, C.I. Steefel; "Dissolution and precipitation kinetics of kaolinite at $25^{\circ} \mathrm{C}$ and $\mathrm{pH} 4$," to be submitted to Geochimica et Cosmochimica Acta.

Steefel, C.I., "Geochemical Kinetics and Transport," Kinetics of Water-Rock Interaction (S. Brantley and J. Kubicki, eds.) in press.

Steefel, C.I., Navarre-Stichler, A., Yang, L., Tomutsa, L.: “A percolation/diffusion threshold model for chemical weathering," to be submitted to Geochimica et Cosmochimica Acta.

EG-Denes LB05010 Novel Imaging Detectors for Materials and Biology Research

P. Denes, "Charge-Coupled Devices, Workshp on Detector Development for Synchrotron Facilities," presentation at Argonne, Dec. 2005.

P. Denes, "Detector Development for SR Applications at LBNL," presentation given at Advanced Photon Source Detector Workshop, Argonne, Jul. 2006.

P. Denes, "High_DQE Detectors for Electron Microscopy," presentation at $6^{\text {th }}$ "Hiroshima Conference on Silicon Tracking Detectors, Carmel, Sep. 2006.

P. Denes et al.; "CMOS Active Pixel Sensors for Electron Microscopy," submitted to Nucl. Inst. and Meth. in Physics Research A.

EETD-Fischer LB05012 Constraining Ammonia Emission Inventories for Control of Air Quality

M.L. Fischer, T.M. Van Reken; "Ammonia Measurements at the T1 Site during MILAGRO," presented at 1st MILAGRO Science Meeting, October 23, 2006.

M.L. Fischer, T.M. Van Reken, D. Littlejohn; "Measurements of $\mathrm{NH}_{3}$ at the T1 Site during MILAGRO," draft to be submitted to Atmospheric Chemistry and Physics.

M.L. Fischer, D. Littlejohn; "Measurements of $\mathrm{NH}_{3}$ Concentration and Flux at a California Forest," draft to be submitted to Atmospheric Environment.

EETD-Johnson LB05017 Highly Efficient PLED Through Polymer Morphology Control

T. Zhang and G. Liu; "Investigation into the Polymer-Small Molecule Blends Film Morphology on Electroluminescence," presented at The $232^{\text {nd }}$ American Chemical Society National Meeting, Sept. 10-14 2006, San Francisco.

T. Zhang and G. Liu; "Investigation into the Polymer-Small Molecule Blends Film Morphology on Electroluminescence," Polymer Preprints (American Chemical Society, Division of Polymer Chemistry) 47(2), 1015 (2006). 
EETD-Newman LB05028 Analysis of High-Temperature Polymer-electrolyte Fuel-cell Phenomena

A. Weber and J. Newman; "Simulation of high-temperature polymer-electrolyte fuel cells," draft to be submitted to Journal of the Electrochemical Society.

EETD-Sathaye LB04043 World Energy Scenarios: The Crucial Role of Energy Demand

M.D. Levine, J. Sathaye, L. Price, J. Sinton, S. de la Rue du Can, N. Zhou, E. Worrell, E.; "Disaggregation of Greenhouse Gas Emissions Scenarios: Matching End-Use Technological Information to Projected Trends," LBNL -58123.

L. Price, S. de la Rue du Can, J. Sinton, and E. Worrell; "Sectoral Trends in Global Energy Use and Greenhouse Gas Emissions," LBNL-56144.

N. Zhou, D. Fridley, J. Lin, M. Levine, J. Sathaye, L. Price, J. Sinton, S. de la Rue du Can; "Energy Use in China: Sectoral Trends and Future Outlook," LBNL-61904.

M. McNeill and V. Letschert V; "Residential Electricity Forecasting using a Bottom-Up Appliance Diffusion Model," draft LBNL - 62055.

J. Sinton, L. Price and A. Pigneri; "Global Energy Database for Scenario Analysis: Sector Modeling Approaches and Associated Data Sheets," LBNL-57274 (2005).

J. Sathaye, "Bottom-up Modeling of Energy and Greenhouse Gas Emissions: Approaches, Results, and Challenges to Inclusion of End-use Technologies," book chapter in M. Schlesinger (Chief Editor) Human-Induced Climate Change: An Interdisciplinary Assessment, Cambridge University Press (in press).

GN-Hugenholtz LB06010 Metagenomics-Enabled Analysis of Termite Hindgut Microbiota for Biomass Conversion and Cleaner Energy

P. Hugenholtz; "Metagenomic Analysis of the Termite Hindgut Implicates Spirochetes and Fibrobacteres as Key Catalysts in Lignocellulose Hydrolysis," in preparation.

GN-Pennacchio LB06019 Development of Cost Effective Sequence-Based Technologies to Identify Genomic Alterations in Cancer

S Volik, B Raphael, G Huang, M Stratton, G Bignel, J Murnane, J Brebner, K Bajsarowicz, P Paris, Q Tao, D Kowbel, A Lapuk, D Shagin, I Shagina, J Gray, J Cheng, P de Jong, P Pevzner, C Collins; "Decoding the Fine-Scale Structure of a Breast Cancer Genome and Transcriptome," Genome Res. 16(3):394-404 (March, 2006).

LSD-Auer LB04010 Molecular Microscopy and Tomography

K.L. McDonald \& M. Auer; "High pressure freezing, cellular tomography and structural cell biology," Biotechniques 41: 137, 139, 141 (2006).

M. Auer, A.J. Koster, U. Ziese, N. Volkmann, C. Bajaj, D.N. Wang, A.J. Hudspeth; "3D architecture of hair cell stereociliar extracellular links as revealed by electron tomography," submitted to $J$. Neuroscience.

J.A. Schaber, W.J. Triffo, S.J. Suh, J. Oliver, M.C. Hastert, J.A. Griswold, M. Auer, A.N. Hamood, K.P. Rumbaugh; "Pseudomonas aeruginosa forms Biofilms in Acute Infection Independently of Cell-to-Cell Signaling," submitted to Infection and Immunity.

K.H. Downing, H. Sui, M. Auer; "Electron tomography - a new, 3D-view of the subcellular world," to be submitted to JACS. 
M. Auer, H. Palsdottir, W.J. Triffo, K.L. McDonald, A.J. Hudspeth; "Visualization of hair cell stereocilia lipid rafts by electron tomography," in preparation for submission to Science.

H. Palsdottir, J.P. Remis, C. Schaudinn, R. Lux, W Shi, K.L. McDonald, J.W. Costerton, M. Auer; "Molecular Organization of Myxococcus xanthus biofilms as revealed by EM tomography," in preparation for submittion to J. Bact.

\section{LSD-Celniker LB05005 Modeling Human Disease In Drosophila melanogaster}

M. Markstein, C. Pitsouli, C. Villalta, S. Celniker, N. Perrimon; "Measuring and Exploiting Position Effects to Maxmize Transgene Regulation," to be submitted to Science.

B. Fisher, B. Pfeiffer, R. Weiszmann, P. Spellman, J. Gray and S. Celniker; "Cell lethal mutations associated with the Drosophila homolog of CRK-7," to be submitted to Drosophila Information Service.

LSD-Conboy LB05009 Unmasking the Human Genome Alternative Splicing Program

T. Clark, A. Schweitzer, D. Das, S. Minovitsky, H. Marr, I. Dubchak, J. Blume, J. Conboy; "Microarray and Computational Analyses Implicate Fox1 as a Major Regulator of Alternative Splicing in Muscle," to be published in proceedings of Alternative Transcript Diversity Symposium II (Heidelberg, Germany; Mar. 21-23, 2006).

D. Das, T. Clark, A. Schweitzer, H. Marr, S. Minovitsky, I. Dubchak, J. Blume, J. Conboy; "Computational analysis of intron regulatory motifs implicates Fox and CELF proteins as major regulators of the muscle-specific alternative splicing program in vertebrate genomes," in preparation for possible submission to J. Nucleic Acids Research.

LSD-Glaeser LB05042 Improved Phase Contrast for Cryo-EM Biological Machines and

Subcellular Structure

R. Cambie, K.H. Downing, D. Typke, R.M. Glaeser, J. Jin; "Design of a Microfabricated, Two-electrode Phase-contrast Element Suitable for Electron Microscopy,"Ultramicroscopy (in press).

LSD-Jagust LB04014 Neuroimaging with Advanced Molecular Probes

G.D. Rabinovici, A.J. Furst, J.P. O’Neil, C.A. Racine, E.C. Mormino, S.L. Baker, S. Chetty, P. Patel, T.A. Pagliaro, W.E. Klunk, C.A. Mathis, H.J. Rosen, B.L. Miller, W.J. Jagust; "11C-PIB PET imaging in Alzheimer disease and frontotemporal lobar degeneration," Neurology (in press).

LSD-Schild LB06022 Determining if PIR51 is a Potential Tumor Suppressor Gene Similar to $\underline{B R C A 2}$

O.V. Kovalenko, C Wiese, and D Schild; "RAD51AP2, a Novel Vertebrate- and MeioticSpecific Protein, Shares a Conserved RAD51-Interacting C-Terminal Domain with RAD51AP1/PIR51," Nucleic Acids Res. 34, 5081-5092 (2006); http://nar.oxfordjournals.org/cgi/content/full/34/18/5081 
C. Wiese, T. Groesser, D. W. Collins, B. Rydberg and D. Schild; "The Human

RAD51AP1/PIR51 Protein Is Required for Homologous Recombination and Genomic Stability," submitted to J. of Biological Chemistry.

MSD-Balsara LB06002 Electron Microscopy of Soft Matter in Two and Three Dimensions

M. Singh, O. Odusanya, G.M. Wilmes, E.D. Gomez, A.J. Patel, H.B. Eitouni, P. Fragouli, H. Iatrou, N. Hadjichristidis, N.P. Balsara; "Decoupling the Mechanical and Electrical Properties of Polymer Electrolytes," to be submitted to Macromolecules.

MSD-Dahmen LB04007 Technique Development for Aberration-Corrected Microscopy at NCEM

M. Herrera, Q. Ramasse, D. Gonzalez, R. Garcia, N. Browning, M. Hopkinson and H.Y. Liu; Microscopy and Microanalysis 12, Suppl. S02, p. 754; for Proceedings of the MSA 2006 Conference, Chicago.

M. Herrera, Q. Ramasse, D. Gonzalez, R. Garcia, N. Browning, M. Hopkinson and H.Y. Liu; Proceedings of the 16th International Microscopy Congress, IMC 2006, Sapporo.

Q. Ramasse, A. Bleloch, R. Klie and N. Browning; Microscopy and Microanalysis 12, Suppl. S02, p. 1342; for Proceedings of the MSA 2006 Conference, Chicago.

MSD-Isacoff LB05016 Light-activated and Regulated Ion Channels and Transporters

P. Gorostiza, M. Volgraf, R. Numano, S. Szobota, D. Trauner, and E.Y. Isacoff, E.Y; "Engineering a channel protein for remote control: mechanisms of photoswitch conjugation and light activation of an ionotropic glutamate receptor," submitted to Neuron.

S. Szobota, P. Gorostiza, M. Volgraf, D. Fortin, O. Tulyathan, K. Kolstad, R. Numano, H. Aaron, S. Ruzin, J. Flannery, R.H. Kramer, D. Trauner, and E.Y. Isacoff; "Remote control of neuronal activity with a light-gated glutamate receptor," submitted to PNAS.

M. Volgraf, P. Gorostiza, R. Numano, R.H. Kramer, E.Y. Isacoff, and D. Trauner, D.; "Allosteric Control of an Ionotropic Glutamate Receptor With an Optical Switch," Nature Chem. Bio. 2, 47-52 (2006).

M. Volgraf, P. Gorostiza, S. Szobota, M.R. Helix, E.Y. Isacoff, D. Trauner; "Reversibly Caged Glutamate: A Photochromic Agonist of Ionotropic Glutamate Receptors," J Am Chem Soc. (in press).

J.J. Chambers, M.R. Banghart, D. Trauner, R.H. Kramer; "Light-induced depolarization of neurons using a modified Shaker $\mathrm{K}(+)$ channel and a molecular photoswitch," $J$ Neurophysiol. 96(5):2792-6 (Nov. 2006).

MSD-Kaindl LB06011 Terahertz-Frequency Conductivity and Ultrafast Optical Excitations in Single-Walled Carbon Nanotubes

J. Wang, R. A. Kaindl, N. C. Nielsen, D. S. Chemla, Y.-Z. Ma, M. W. Graham, and G. R. Fleming; "Photoinduced Mid-Infrared Excitations in Single-Walled Carbon Nanotubes," in preparation.

R. A. Kaindl, B. A. Schmid, R. Huber, D. S. Chemla, Y.-Z. Ma, M. W. Graham, and G. R. Fleming; "Ultrafast THz Dynamics in Single-Walled Carbon Nanotubes," in preparation. 
Y.-Z. Ma, M. W. Graham, M. A. Prantil, A. J. Van Tassle, and G. R. Fleming; "Femtosecond Mid-Infrared Spectroscopy of Single-Walled Carbon Nanotubes," in preparation.

Y.-Z. Ma, M. W. Graham, and G. R. Fleming; "Three-Pulse Photon Echo Peak Shift Spectroscopy of Single-Walled Carbon Nanotubes Embedded in Various Matrixes," in preparation.

MSD-Lanzara LB06029 Carbon Based Materials for Renewable Energy

S.Y. Zhou SY, G.H. Gweon GH, J. Graf, A. V. Fedorov, C. D. Spataru,R. D. Diehl, Y. Kopelevich, D.-H. Lee, Steven G. Louie, and A. Lanzara; "First direct observation of Dirac fermions in graphite'," Nature Physics 2, 595 (2006).

$\mathrm{H} \mathrm{Fu}$, and D.-H. Lee, "The Image of a Unit Charge above Graphene," in preparation.

MSD-Orenstein/ Ramesh LB05045 Science and Technology of Quantum Materials

L. W. Martin et al.; "Epitaxial PbVO3: A new multiferroic system," Applied Physics Letters (in press).

H. Fu, C. Honerkamp and D.-H. Lee; "A Renormalization Group Study of the Electronphonon Interaction in High T_c Cuprates," Euro. Phys. Lett. (in press).

S.Y. Zhou SY, G.H. Gweon GH, J. Graf, A. V. Fedorov, C. D. Spataru,R. D. Diehl, Y. Kopelevich, D.-H. Lee, Steven G. Louie, and A. Lanzara; "First direct observation of Dirac fermions in graphite," Nature Physics 2, 595 (2006).

H. Fu and D.-H. Lee; "On the Dichotomy between the Nodal and Antinodal Excitations in High-temperature Superconductors," Phys. Rev. B (in press).

J.X. Li, C.Q. Wu, and D.-H. Lee; "Checkerboard charge density wave and pseudogap in high-Tc cuprates," Phys. Rev. B (in press).

T.C. Ribeiro, A. Seidel, J.H. Han, and D.-H. Lee; "The Electronic States of Two oppositely doped Mott Insulators Bilayers," accepted in Euro. Phys. Lett.

Haimei Zheng, Qian Zhan, Florin Zavaliche, Matt Sherburne,Florian Straub, Maria P. Cruz, Long-Qing Chen, Uli Dahmen, and R. Ramesh; "Controlling Self-Assembled PerovskiteSpinel Nanostructures," Nanoletters 6, 1401(2006).

Haimei Zheng, Florian Straub, Qian Zhan, Pei-Ling Yang, Wen-Kuo Hsieh, Florin Zavaliche, Ying-Hao Chu, Uli Dahmen and R. Ramesh: "Self-assembled Growth of BiFeO3-CoFe2O4 Nanostructures," Advanced Materials (in press).

T. Zhao, A. Scholl, F. Zavaliche, K. Lee, M. Barry, A. Doran, M. P. Cruz, Y. H. Chu, C. Ederer, N. A. Spaldin, R. R. Das, D. M. Kim, S.H. Baek, C. B. Eom and R. Ramesh; "Electrical control of antiferromagnetic domains in multiferroic $\mathrm{BiFeO} 3$ film at room temperature," Nature Materials (in press).

R. Ramesh and N. Spaldin; "Multiferroic Thin Films and Heterostructures: Progress and Prospects," review in Nature Materials (2006).

Q.Zhan, R. Yu, S.P. Crane, H. Zheng, C. Kisielowski, R. Ramesh "Structure and interface chemistry of perovskite-spinel nanocomposite thin films," Appl. Phys. Lett. (in press).

F. Zavaliche, et al.; Phase Transitions 797, 1-27 (2006).

Y.H. Chu et al.; Advanced Materials 18, 2306 (2006).

MSD-Pines LB06034 Polarizer System for Microfluidics

E. Harel, C. Hilty, K. Koen, E. McDonnell, and A. Pines; "Time-of-flight flow imaging of two-component flow inside a microfluidic chip," accepted in Physical Review Letters. 
M. S. Anwar, C. Hilty, C. Chu, L.S. Bouchard, K. Pierce and A. Pines: "Spin coherence transfer in chemical transformations monitored by remote detection NMR," submitted to Analytical Chem.

I.V. Koptyug, K.V. Kovtunov, S. Burt, M. S. Anwar, C. Hilty, A. Pines, R.Z. Sagdeev; "Para-hydrogen induced polarization in heterogeneous hydrogenation reactions," submitted to J. Amer. Chem. Soc.

MSD-Ritchie LB06020 Aging, Disease and the Mechanical Response of Biological Tissues, Specifically Human Bone

J. W. Ager III, G. Balooch, and R. O. Ritchie; "Fracture, aging and disease in bone," J. Materials Res. 21, 1878-92 (2006).

J. W. Ager III, R. K. Nalla, G. Balooch, G. Kim, M. Pugach, S. Habelitz, G. W. Marshall, J. H. Kinney, and R. O. Ritchie; "On the increasing fragility of human teeth with age: A deep-ultraviolet resonance Raman study," J. Bone Min. Res. (in press). DOI: 10.1359/jbmr.060816

R. O. Ritchie, R. K. Nalla, J. J. Kruzic , J. W. Ager III, G. Balooch, and J. H. Kinney; "Fracture and aging in bone: Toughness and structural characterization," Strain 42, 225 232 (2006).

G. Balooch, W. Yao, J. W. Ager, M. Balooch, R. K. Nalla, A. E. Porter, A. Mastroianni, R. O. Ritchie, and N. E. Lane; "The aminobisphosphonate, risedronate, preserves bone mineral and mechanical properties caused by glucocorticoid-induced osteoporosis in mouse and human bone," submitted to New Eng. J. Medicine.

W. Yao, Z. Cheng, K. J. Koester, J. W. Ager, G. Balooch, M. Balooch, M. Ratnarathorn, J. Teung, A. Pham, C. Busse, R. O. Ritchie, and N. E. Lane; "The distribution and homogeneity of bone mineral is maintained with intravenous bisphosphonates in aged estrogen deficiency rats by a mechanism that involves the TGF/Smad signaling," submitted to J. Bone Min. Res.

J. W. Ager III, H. Barth, G. Kim, P. Fischer, G. Balooch, K. J. Koester, A. E. Porter, J. H. Kinney, and R. O. Ritchie; "Soft x-ray microscopy of human dentin with sub-100 nm resolution," in preparation for submission to J. Structural Biology.

MSD-Somorjai LB06023 Electron Glow Generated by Gas Phase Exothermic Catalytic Reactions Using Metal-Semiconductor Nanodiodes

J. Y. Park and G. A. Somorjai; "The Catalytic Nanodiode: Detecting Continuous Electron Flow at Oxide-Metal Interfaces Generated by Gas Phase Exothermic Chemical Reaction," Chem. Phys. Chem.7, 1409-1413 (2006). http://www3.interscience.wiley.com/cgi-bin/abstract/112641547/

J. Y. Park and G. A. Somorjai; "Energy Conversion from Catalytic Reaction to Hot Electron Current with Metal - Semiconductor Schottky Diodes," J. Vac. Sci Technol. B 24 (4) 1967 (2006). http://scitation.aip.org/journals/doc/JVTBD9-ft/vol_24/iss_4/1967_1.html

G. A. Somorjai, K. M. Bratlie, M. O. Montano and J. Y. Park; "Dynamics of Surface Catalyzed Reactions; the roles of Surface Defects, Surface Diffusion and Hot Electrons," J. Phys. Chem. B 110, 20014-20022 (2006). http://pubs.acs.org/cgibin/abstract.cgi/jpcbfk/2006/110/i40/abs/jp062569d.html

J. Y. Park, J. R. Renzas, A. M. Contreras and G. A. Somorjai; "The Genesis and Importance of Oxide-Metal Interface Controlled Heterogeneous Catalysis; The Catalytic Nanodiode," Topics in Catalysis (in press). 
G. A. Somorjai, F. Tao, J. Y. Park; "The Nanoscience Revolution: Merging of Colloid Science, Catalysis and Nanoelectronics," submitted to Topics in Catalysis.

MSD-Souza/ Louie LB05035 Computational and Theoretical Studies of Bulk and Nano Solid Systems

X. Wang, J. R. Yates, I. Souza, and D. Vanderbilt; "Ab-initio calculation of the anomalous Hall conductivity by Wannier interpolation," Physical Review B 74, 195118 (21 November 2006). http://link.aps.org/abstract/PRB/v74/e195118

F. Giustino, J.; R. Yates, I. Souza, M. L. Cohen, and S. G. Louie; "Electron-phonon interaction via electronic and lattice Wannier functions: Superconductivity in borondoped diamond reexamined," submitted to Physical Review Letters.

E. Roman, J. R. Yates, M. Veithen, D. Vanderbilt, and I. Souza; "Ab initio study of the nonlinear optics of III-V semiconductors in the terahertz regime," Physical Review B (in press).

J. R. Yates, X. Wang, D. Vanderbilt, and I. Souza; "Spectral and Fermi surface properties from Wannier interpolation," in preparation for submission to Phys. Rev. B.

MSD-Vishwanath LB05038 Experimental Signatures of Deconfined Phases and Transitions

Fa Wang and A. Vishwanath; "Spin Liquid States on the Triangular and Kagome Lattices: A Projective Symmetry Group Analysis of Schwinger Boson States," Phys. Rev. B 74, 174423 (2006).

L. Mathey, E. Altman, A. Vishwanath; "Nernst effect and diamagnetism in phase fluctuating superconductors," Cond-mat/0612096 (preprint), submitted to Physical Review Letters.

Fa Wang and F. Y. Wu; "Exact solution of close-packed dimers on the kagome lattice," Cond-mat/0612573 (preprint), submitted to Physical Review Letters.

NSD-Lesko LB04017 Research and Development for Double Beta Decay (DBD) Experiments

R. Henning et al; "Study of a Highly-segmented HPGe Detector at the Oroville LowBackground Counting Facility, APS meeting, Dallas, April 2006.

R. Henning for MaGe Collaboration, "'MaGe:' Simulation Framework for 76Ge-based Neutrinoless Double-beta Decay Experiments," Neutrino 2006 poster presentation, Santa $\mathrm{Fe}, \mathrm{NM}$, June 2006.

R. Henning for MaGe collaboration.; "'MaGe,' a Simulation Framework for Germaniumbased Neutrinoless Double- Beta Decay Experiments," IEEE NSS Conference, San Diego, CA, Oct 2006.

Cuoricino collaboration; "Cuoricino and CUORE detectors: developing big arrays of large mass bolometers for rare events physics," Nuclear Physics B - Proceedings Supplements 150, 214-218 (January 2006).

Cuoricino collaboration; "Further developments in the CUORICINO experiment," Nuclear Instruments and Methods in Physics- Research Section A: Accelerators, Spectrometers, Detectors and Associated Equipment 559 (2), 352-354 (15 April 2006).

NSD-McMahan LB05026 New Capabilities in Nuclear Astrophysics and Radiation Biology Using Neutrons at the 88-Inch Cyclotron

D. Bleuel, M. McMahan, L. Ahle, B. Barquest, J. Cerny, L. Heilbronn, C. Jewett; "Characterization of a Tunable Quasi-Monoenergetic Neutron Beam from Deuteron 
Breakup," Conference on Applications of Accelerators for Research and Industry 2006, July 2006 (in press).

NSD-Phair LB05030 Improved Spectroscopy of Weakly Bound States in Nuclei

R. M. Clark, L. W. Phair, M. Descovich, M. Cromaz, et al; "Population of nuclei via ${ }^{7} \mathrm{Li}$ induced binary reactions," Phys. Rev. C 72, 054605 (2005).

J. T. Burke, L. A. Bernstein, J. Escher, L. Ahle, et al; "Deducing the ${ }^{237} \mathrm{U}(n, f)$ cross section using the Surrogate Ratio Method," Phys. Rev. C 73, 054604 (2006).

C. W. Beausang, S. R. Lesher, J. T. Burke, L. A. Bernstein, et al; "New Results on Fission Cross Sections in Actinide Nuclei using the Surrogate Ratio Method and on Conversion Coefficients in Triaxial Strongly Deformed Bands in ${ }^{167} \mathrm{Lu}$ from ICE Ball and Gammasphere," accepted in Acta Physica Polonica B.

L. A. Bernstein, J. T. Burke, L. Ahle, J. A. Church, et al; "Deducing the ${ }^{237} \mathrm{U}(n, g)$ and $(n, 2 n)$ cross-sections using a new Surrogate Ratio Method," submitted to Phys. Rev. C.

NSD-Vetter LB06025 Conceptual Study for a Novel Nuclear Astrophysics Accelerator Capablity to Measure Nuclear Reactions That Power the Stars

D.S. Todd, D. Leitner, M. Leitner, R. Maruyama,P.A. Vetter, K.N. Xu; "Design of the Low Energy Astrophysics Research Facility CLAIRE,“ Nuclear Instruments and Methods A (in press).

PBD-Crooks LB05044 Statistical Dynamics of Protein Evolution

G. E. Crooks, "Nonequilibrium maximum-entropy hyperensembles: Beyond BoltzmannGibbs statistics," submitted to Phys. Rev. Lett., eprint: cond-mat/060312.

PBD-Fletcher LB05013 Synthetic Cytoskeleton: Protrusive Structure for Reconstituted Cell Motility

A. P. Liu and D. A. Fletcher; "Actin polymerization serves as a membrane domain switch in model lipid bilayers," Biophysical Journal 91 (11), 4064-70 (2006).

C. M. Nelson, M. M. van Duijn, J. L. Inman, D. A. Fletcher, and M. J. Bissell; "Tissue geometry determines sites of branching morphogenesis in organotypic cultures," Science 314 (5797), 298-300 (2006).

PBD-Frei LB06006 Visible Light-driven Water Oxidation in Mesoporous Solids

R. Nakamura and H. Frei; "Visible Light-Driven Water Oxidation by Ir Oxide Clusters to Single Cr Centers in Mesoporous Silica," J. Am. Chem. Soc. 128, 10668 (2006).

W.W. Weare, H. Han, L. Andersen, Y. Pushkar, V.K. Yachandra, and H. Frei; "Coupling of Biomimetic Oxygen-Evolving Mn Dimer Clusters to Cr Charge Transfer Chromophore on Nanoporous Silica Support," in preparation.

PBD-Groves LB06008 Magnetic Control of Spatial Organization at Bio-Membranes

A.R. Sapuri-Butti, Q. Li, J.T. Groves, A.N. Parikh; "Nonequilibrium patterns of cholesterolrich chemical heterogenieties within single fluid supported phospholipid bilayer membranes," Langmuir 22, 5374 (2006). 
Groves et al; invited review on this general area for Soft Matter (in press).

PBD-Liphardt LB05021 Rapid Characterization of Microbial RNAs Using Artificial Nanopores

E. Trepagnier, A. Radenovic, J. Liphardt; "Kinetics of Polymer Threading through Artificial Nanopores," to be submitted to Phys. Rev. Lett.

E. Trepagnier, A. Radenovic, J. Liphardt; "Mechanical Unfolding of Single RNA IRES Elements Through Artificial Nanopores," to be submitted to Nature Biotechnology.

PD-Battaglia LB05002 Advanced Detectors to Understand the Early Universe

M. Battaglia et al.; "Development Of Monolithic Cmos Pixel Sensors For The Ilc At LBNL," to appear in the Proceedings of International Symposium on Detector Development for Particle, Astroparticle and Synchrotron Radiation Experiments (SNIC 2006), Menlo Park, California, 3-6 Apr 2006.

M. Battaglia, B. Hooberman, D. Contarato, L. Greiner, T. Kim and H. Wieman; "BackThinning Of Cmos Monolithic Active Pixel Sensors For The Ilc At LBNL," to appear in the Proceedings of International Symposium on Detector Development for Particle, Astroparticle and Synchrotron Radiation Experiments (SNIC 2006), Menlo Park, California, 3-6 Apr 2006.

M. Battaglia; "Response Simulation of CMOS Pixel Sensors for the ILC Vertex Tracker," Nucl. Instrum. and Meth. A (in press).

M. Battaglia, D. Contarato, P. Giubilato, L. Greiner, L. Glesener and B. Hooberman; “A study of monolithic CMOS pixel sensors back-thinning and their application for a pixel beam telescope," Nucl. Instrum. and Meth. A (in press). arXiv:physics/0611081.

PD-Heeger LB04012 Designing a Novel Reactor Neutrino Oscillation Experiment for Measuring the Unknown Mixing Angle Theta-13

L.J. Wen, J. Cao, K.B. Luk, Y.Q. Ma, Y.F. Wang, C.G. Yang; "Measuring Cosmogenic ${ }^{9} \mathrm{Li}$ Background in a Reactor Neutrino Experiments," Nucl. Instrument. Method A564, 471 (2006).

X.H. Guo et al.; "Daya Bay Project," submitted to Office of High Energy Physics, Department of Energy, Oct 1, 2006.

PD-Murayama LB05027 New Directions for Theoretical Physics at the TeV-Scale

Raphael Bousso, Ben Freivogel; "A Paradox in the global description of the multiverse," Oct 2006. e-Print Archive: hep-th/0610132

Raphael Bousso, Ben Freivogel, I-Sheng Yang; "Eternal Inflation: The Inside Story," Phys. Rev. D 74, 103516 (2006); UCB-PTH-06-09, LBNL-60250 (Jun 2006). e-Print Archive: hep-th/0606114

Raphael Bousso; "Holographic probabilities in eternal inflation," Phys. Rev. Lett. 97, 191302 (2006); UCB-PTH-06-10, LBNL-60251 (May 2006). e-Print Archive: hep-th/0605263, Abstract and Postscript and PDF from arXiv.org

Raphael Bousso, Ben Freivogel, Matthew Lippert, "Probabilities in the landscape: The Decay of nearly flat space," Phys. Rev. D 74, 046008 (2006). e-Print Archive: hep-th/0603105

Christian W. Bauer, Matthew D. Schwartz; "Event Generation from Effective Field Theory," Jul 2006. e-Print Archive: hep-ph/0607296 
Christian W. Bauer, Matthew D. Schwartz; "Improving jet distributions with effective field theory," Phys. Rev. Lett. 97, 142001 (2006), LBNL-59964. e-Print Archive: hep$\mathrm{ph} / 0604065$

Sergio Ferrara, Eric G. Gimon, Renata Kallosh; "Magic supergravities, N= 8 and black hole composites," CERN-PH-TH-2006-116, SU-ITP-2006-19, LBNL-60487, UCLA-06-TEP19 (Jun 2006). e-Print Archive: hep-th/0606211

Cross-Div.-Lester LB05020 Development and Application of Quantum Monte Carol (QMC) Methods to Biological Systems

W. A. Lester, Jr. and R. Salomon-Ferrer; "Some Developments in Quantum Monte Carlo for Electronic Structure: Methods and Application to a Bio System," THEOCHEM 771, 51 (2006).

B. Austin, A. Aspuru-Guzik, R. Salomon-Ferrer, and W. A. Lester, Jr.; "Linear-Scaling Evaluation of the Local Energy in Quantum Monte Carlo," in Proceedings of the Pacifichem Symposium on Advances in Quantum Monte Carlo, Oxford University Press (in press). 
